\title{
Mass angular momentum inequality for axisymmetric vacuum data with small trace
}

\author{
XIN ZHOU
}

In this paper, we prove the mass angular momentum inequality $[12,15,23]$ for axisymmetric, asymptotically flat, and vacuum data sets with small trace. Given an initial data set with small trace, we construct a boost evolution spacetime of the Einstein vacuum equations as [10]. Then a perturbation method is used to solve the maximal surface equation in the spacetime under certain growing condition at infinity. When the initial data set is axisymmetric, we get an axisymmetric maximal graph with the same mass and angular momentum as the given data. The inequality follows from the known results $[12,15,23]$ about the maximal graph.

\section{Introduction}

Based on the gravitational collapse pictures [14], it is conjectured that the angular momentum should be bounded by the mass for physically reasonable solutions of the Einstein equations. It is true for Kerr black hole solutions which are stationary. For dynamical, axisymmetric solutions some progresses have been made over the past few years. Dain [15] first proved such an inequality for Brill data (see [15, Definition 2.1]), which is a special class of axisymmetric, maximal, and asymptotically flat vacuum data. Later, Chruściel, Li and Weinstein $[11,12]$ generalized it to a class of axisymmetric, maximal data admitting an Ernst potential with positive mass density, and certain asymptotically flatness conditions. Recently Schoen and Zhou [23] gave a simplified proof for more general asymptotic conditions and an $L^{6}$ norm bound.

All the existing results require the solutions to be maximal, which restricts the data to be a special time-slice in a spacetime. However it should be 
unnecessary according to the gravitational collapse pictures ${ }^{1}$. It is natural and interesting to study the non-maximal case. In this paper, we will prove the mass angular momentum inequality for non-maximal vacuum data with small trace by exploring the Einstein equations and a perturbation method. Using notations in Section 1.2, our main theorem is.

Theorem 1.1 (Main Theorem 1). Suppose $(\Sigma, e)$ is a simply connected 3-manifold, which is Euclidean at infinity with two ends and axisymmetric in the sense of Definition 1.3. Given an asymptotically flat, axisymmetric vacuum data $(g, k) \in \mathcal{V C}_{s+2, \delta+\frac{1}{2}}^{a}(\Sigma)$ (see Definition 1.5) with $s \in \mathbb{N}, s \geq 7$, $\delta \in \mathbb{R},-\frac{3}{2}<\delta<-1$, if $\left\|t r_{g} k\right\|_{H_{s-2, \delta+\frac{3}{2}}(\Sigma)} \leq \epsilon$ with $\epsilon$ given in Theorem 1.6, we have

$$
m \geq \sqrt{|J|}
$$

where $m$ and $J$ are the ADM (abbreviated for Arnowitt-Deser-Misner, see page 193 [24]) mass (1.6) and angular momentum (1.8) of $(\Sigma, g, k)$ respectively.

Our method comes from a question suggested by R. Schoen:

(Q): Is there a canonical way to deform a non-maximal, axisymmetric, vacuum data to a unique maximal, vacuum data with the same physical quantities, i.e., the mass and angular momentum, which also preserves the axially symmetry?

A definite answer of the above question will imply the mass angular momentum inequality in the non-maximal case. In fact, there are already some works about the deformation of vacuum constraint equations (VCEs) [4, 13]. But it is hard to maintain the symmetries and physical quantities at the same time. So the main difficulty is to maintain the symmetries and the physical quantities simultaneously when deforming the VCEs. We overcome this difficulty by using certain conversation laws of the Einstein equations.

\subsection{General relativity backgrounds}

In Einstein's theory for General Relativity ${ }^{2}$, we use $\left(\mathcal{V}^{3,1}, \gamma\right)$ to denote a spacetime, where $\mathcal{V}^{3,1}$ is a 4 -dimensional oriented smooth manifold, and $\gamma$ is

\footnotetext{
${ }^{1}$ The axisymmetric condition is indeed necessary, since otherwise vacuum counterexamples were constructed by Huang, Schoen and Wang [18].

${ }^{2}$ We refer to [24] for all the concepts.
} 
a Lorentzian metric of signature $(3,1)$. The Einstein equation, which predicts the evolution of the spacetime, is given by

$$
R i c_{\gamma}-\frac{1}{2} R_{\gamma} \gamma=8 \pi T
$$

where $R i c_{\gamma}$ is the Ricci curvature of $\gamma$, and $R_{\gamma}$ the scalar curvature of $\gamma$. $T$ is the stress-energy tensor. In the vacuum case, $T \equiv 0$, so the Einstein vacuum equation, abbreviated as (EVE) in the following, reduces to

$$
R i c_{\gamma}=0
$$

A vacuum constraint initial data set or abbreviated as vacuum data for the EVE is a triple $(\Sigma, g, k)$, where $\Sigma$ is a connected complete 3 -dimensional manifold, $g$ a Riemannian metric, and $k$ a symmetric two tensor on $\Sigma$, satisfying the vacuum constraint equations, abbreviated as (VCE),

$$
\left\{\begin{array}{l}
R_{g}-|k|_{g}^{2}+\left(t r_{g} k\right)^{2}=0 \\
\operatorname{div}_{g}\left(k-\left(t r_{g} k\right) g\right)=0
\end{array}\right.
$$

By the famous initial value formulation for the Einstein equations by $\mathrm{Y}$. Choquet-Bruhat in 1952 (see [9, 24]), we can always think the vacuum data $(\Sigma, g, k)$ as been embedded in some spacetime $(\mathcal{V}, \gamma)$ satisfying (EVE), where $g$ is the restriction of $\gamma$ to $\Sigma$, and $k$ is the second fundamental form of the embedding.

$(\Sigma, e)$ is called Euclidean at infinity, where $e$ is a Riemannian metric on $\Sigma$, if there is a compact subset $\Sigma_{\text {int }} \subset \Sigma$, such that the complement $\Sigma_{\text {ext }}=$ $\Sigma \backslash \Sigma_{\text {int }}$ is a disjoint union of finitely many open sets $\Sigma_{\text {ext }}=\cup_{i} E_{i}$, and each $E_{i}$ is diffeomorphic to $\mathbb{R}^{3}$ cutting off a ball $B_{R}$, and on each $E_{i}$, $e$ is the pull back of the standard Euclidean metric on $\mathbb{R}^{3}$. Here $\Sigma_{\text {int }}$ is called the interior region, $\Sigma_{\text {ext }}$ the exterior region, and each $E_{i}$ an end. Each end $E$ has a coordinate system $\left\{x_{i}: i=1,2,3\right\}$ inherited from $\mathbb{R}^{3}$. Let $r=\sqrt{\sum_{i}\left(x_{i}\right)^{2}}$. $(\Sigma, g, k)$ is said to be asymptotically flat, abbreviated as $(\mathbf{A F})$, if $(\Sigma, e)$ is Enclidean at infinity for some $e$, and there exists an $\alpha>\frac{1}{2}$, such that under coordinates $\left\{x_{i}: i=1,2,3\right\}$,

$$
g_{i j}=\delta_{i j}+O_{2}\left(r^{-\alpha}\right), \quad k_{i j}=O_{1}\left(r^{-1-\alpha}\right) .
$$

Under these conditions, the ADM mass is defined as,

$$
m=\lim _{r \rightarrow \infty} \frac{1}{16 \pi} \int_{S_{r}}\left(g_{i j, i}-g_{i i, j}\right) \nu^{j} d \sigma(r),
$$


where $S_{r}$ is the sphere of radius $r$ in $\mathbb{R}^{3}, g_{i j, k}=\frac{\partial g_{i j}}{\partial x_{k}}$ and $\nu^{j}$ is the Euclidean unit outer normal of $S_{r}$ with $d \sigma(r)$ the surface element of $S_{r}$. The famous positive mass theorem by Schoen and Yau [21, 22] and Witten [25] says that $m \geq 0$ under the dominant energy condition.

If the initial data set $(\Sigma, g, k)$ is axisymmetric (cf. [11, 15]) under an axial Killing vector field $\xi$, i.e.,

$$
\mathcal{L}_{\xi} g=0, \quad \mathcal{L}_{\xi} k=0
$$

where $\mathcal{L}$ denotes the Lie derivative, we also have a well-defined angular momentum $J$ (cf. $[15,24]$ ) of a close 2-surface $S \subset \Sigma$

$$
J(S)=\frac{1}{8 \pi} \int_{S} \pi_{i j} \xi^{i} \nu^{j} d \sigma_{g},
$$

where $\pi_{i j}=k_{i j}-t r_{g}(k) g_{i j}$ is divergence free by (1.4), and $\nu, d \sigma_{g}$ are, respectively the unit outer normal of $S$ and surface element w.r.t. $g$.

\subsection{Ideas and main results}

In this paper, we will prove the mass angular momentum inequality for certain axisymmetric, AF vacuum data $(\Sigma, g, k)$ with small $t r_{g} k$, especially we partially solved the question asked by Schoen. We will use the full Einstein equations and a perturbation method. Given an $\mathrm{AF}$ vacuum data $(\Sigma, g, k)$, we will solve the boost problem of $(\mathrm{EVE})$ for $(\Sigma, g, k)$ as $[3,10]$ to get a spacetime $(\mathcal{V}, \gamma)$, where $\mathcal{V}$ is a subset of $\Sigma \times \mathbb{R}$ which grows linearly at infinity. Given a function $u$ defined on $\Sigma$, the graph $\operatorname{Graph}_{u}=\{(x, u(x)) \in$ $\Sigma \times \mathbb{R}, x \in \Sigma\}$ of $u$ lies inside $\mathcal{V}$, when $|u|$ has roughly sub-linear growth. We want to find a solution to $H_{u}=0$, where $H_{u}$ is the mean curvature of $\operatorname{Graph}_{u}$ w.r.t. $(\mathcal{V}, \gamma)$. Now fix a 3 -manifold $(\Sigma, e)$ Euclidean at infinity, we can construct a mapping $\mathcal{H}$ which takes the triple $(g, k, u)$ to the mean curvature $H_{u}$, i.e., $\mathcal{H}:(g, k, u) \rightarrow H_{u}$. Viewing $(g, k)$ as parameters and $u$ as unknown function, our equation changes to

$$
\mathcal{H}(g, k, u)=0
$$

When $(g, k)$ is maximal, i.e. $t r_{g} k=0, u \equiv 0$ is a solution to (1.9). So we can use the Inverse Function Theorem to solve $\mathcal{H}(g, k, u)=0$ when $\operatorname{tr}_{g} k$ is small enough. From now on, we always assume $s \in \mathbb{N}$ and $\delta \in \mathbb{R}$. Using notations from Section 2, we have 
Definition 1.2. Fix a 3-dimensional manifold $(\Sigma, e)$ which is Euclidean at infinity.

(1) The vacuum constraint data sets $\mathcal{V C}_{s+1, \delta+\frac{1}{2}}(\Sigma)$ is defined to be the set of solutions $(g, k)$ to $(1.4)$, such that $(g-e, k) \in H_{s+1, \delta+\frac{1}{2}}(\Sigma) \times$ $H_{s, \delta+\frac{3}{2}}(\Sigma)$.

(2) The maximal vacuum constraint data sets $\mathcal{M V C}_{s+1, \delta+\frac{1}{2}}(\Sigma)$ is defined to be the subset of $\mathcal{V} \mathcal{C}_{s+1, \delta+\frac{1}{2}}(\Sigma)$ satisfying $\operatorname{tr}_{g} k=0$.

Inside $\mathcal{V C}_{s+1, \delta+\frac{1}{2}}(\Sigma)$ and $\mathcal{M} \mathcal{V} \mathcal{C}_{s+1, \delta+\frac{1}{2}}(\Sigma)$, we use the topology induced by the Sobolev norms of $H_{s+1, \delta+\frac{1}{2}}(\Sigma) \times H_{s, \delta+\frac{3}{2}}(\Sigma)$ as in Definition 2.3.

Definition 1.3. A simply connected 3-manifold $(\Sigma, e)$ which is Euclidean at infinity is called axisymmetric, if

(1) $\Sigma$ is diffeomorphic to $\mathbb{R}^{3}$ minus some points $\left\{a_{k}\right\}_{k=1}^{l-1}$ on the $z$-axis $\Gamma=\left\{(\rho, \varphi, z) \in \mathbb{R}^{3}: \rho=0\right\}$, with one end modeled by a neighborhood of $\infty$, and other ends by a neighborhood of $a_{k}$ with coordinates given by a Kelvin transformation: $\left\{x^{\prime}=\frac{x-a_{k}}{\left|x-a_{k}\right|^{2}}\right\}$;

(2) $\mathcal{L}_{\partial_{\varphi}} e=0$, where $\varphi$ is the azimuth of the cylindrical coordinates $\{\rho, \varphi, z\}$.

Remark 1.4. Near $\infty, e$ is given by the Euclidean metric $d s_{0}^{2}$, and near each puncture $a_{k}, e$ is the pull back of the Euclidean metric by the Kelvin transformation, i.e., $e=\frac{1}{|x|^{4}} d s_{0}^{2}$. In fact, by Chruściel's reduction in [11], any simply connected, axisymmetric, $\operatorname{AF}$ vacuum data $(\Sigma, g)$ has the underlying topology $\Sigma$ given by $\mathbb{R}^{3}$ minus finitely many points on the $z$ axis, with the Killing vector field $\frac{\partial}{\partial \varphi}$.

Definition 1.5. Given $(\Sigma, e)$ as in Definition 1.3.

(1) An initial data set $(g, k)$ is called axisymmetric, if the symmetry conditions (1.7) hold for the Killing vector field $\xi=\frac{\partial}{\partial \varphi}$.

(2) $\mathcal{V C}_{s+1, \delta+\frac{1}{2}}^{a}(\Sigma)$ and $\mathcal{M V C}_{s+1, \delta+\frac{1}{2}}^{a}(\Sigma)$ are the axisymmetric subset of $\mathcal{V} \mathcal{C}_{s+1, \delta+\frac{1}{2}}(\Sigma)$ and $\mathcal{M V C}_{s+1, \delta+\frac{1}{2}}(\Sigma)$ respectively.

The following theorem is one of our main results, which is a summarization of Theorem 4.12, Lemma 4.13, Lemma 4.14 and Theorem 4.16.

Theorem 1.6 (Main Theorem 2). Given $s \geq 4,-2<\delta<-1$.

(i) Let $(\Sigma, e)$ be a 3-dimensional manifold which is Euclidean at infinity. For any $(g, k) \in \mathcal{V} \mathcal{C}_{s+2, \delta+\frac{1}{2}}(\Sigma)$, where $\lambda e \leq g \leq \lambda^{-1}$ e for some $\lambda>0$, there exists a small number $\epsilon$ depending only on $\lambda$ and 
$\|g-e\|_{H_{s+2, \delta+\frac{1}{2}}(\Sigma)}+\|k\|_{H_{s+1, \delta+\frac{3}{2}}(\Sigma)}$, such that if $\left\|t r_{g} k\right\|_{H_{s-2, \delta+\frac{3}{2}}(\Sigma)} \leq \epsilon$, then there exists a spacetime $(\mathcal{V}, \gamma)$ solving the $(E V E)$, and a function $u \in H_{s+2, \delta-\frac{1}{2}}(\Sigma)$ solving the maximal surface equation (1.9) inside $(\mathcal{V}, \gamma)$. The induced metric $g_{u}$ and second fundamental form $k_{u}$ of Graph $_{u}$ satisfy $\left(g_{u}, k_{u}\right) \in \mathcal{M V C}_{s+1, \delta+\frac{1}{2}}(\Sigma)$.

(ii) If $-\frac{3}{2}<\delta<-1$, the ADM mass of $\left(\Sigma, g_{u}, k_{u}\right)$ is the same as that of $(\Sigma, g, k)$.

(iii) If $(\Sigma, e, g, k)$ is simply connected, axisymmetric, then $u$ can be chosen to be axisymmetric, hence $\left(\Sigma, g_{u}, k_{u}\right)$ is axisymmetric, and has the same angular momentum as $(\Sigma, g, k)$.

Remark 1.7. The weight $\delta$ corresponds the decay $g \sim e+O\left(r^{-(\delta+2)}\right)$ and $k \sim O\left(r^{-(\delta+3)}\right)$ by the Sobolev embedding Lemma 2.5. $\left(g_{u}, k_{u}\right)$ is always assumed to be pulled back to $\Sigma$ by the graphical map $F_{u}: x \rightarrow(x, u(x))$.

Remark 1.8. The order of regularity of our final solution $\left(g_{u}, k_{u}\right)$ decreases by 1 than our starting data $(g, k)$. This is due to the fact that the restriction of $H_{s}$-Sobolev functions on a spacetime to a hypersurface decreases the regularity by 1 (see Lemma 2.8).

Our main Theorem 1.1 is then a corollary of the above theorem.

Proof of Theorem 1.1: Let $u$ be the solution given in part (iii) of Theorem 1.6. Then the induced maximal data $\left(g_{u}, k_{u}\right) \in \mathcal{M V \mathcal { C }}_{s+1, \delta+\frac{1}{2}}^{a}(\Sigma)$, and the ADM mass $m$ and angular momentum $J$ of $(g, k)$ and $\left(g_{u}, k_{u}^{2}\right)$ are the same. Now by Sobolev embedding Lemma 2.5, $\left(g_{u}-e, k_{u}\right) \in C_{\beta}^{s-1}(\Sigma) \times$ $C_{\beta+1}^{s-2}(\Sigma)$ for some $\frac{1}{2}<\beta<\delta+2<1$. So $\left(\Sigma, g_{u}, k_{u}\right)$ is an axisymmetric, maximal vacuum data, with asymptotic conditions $g_{u}=\delta+O_{s-1}\left(\frac{1}{r^{\beta}}\right)$ and $k_{u}=O_{s-2}\left(\frac{1}{r^{\beta+1}}\right)$, so the mass angular momentum inequality in [23] holds on $\left(\Sigma, g_{u}, k_{u}\right)$. Hence $m \geq \sqrt{|J|}$.

The paper is organized as follows: in Section 2, we will review the weighted Sobolev space theory covered by $[2,7,8,10]$ and the geometry of hypersurfaces in 3+1-dimensional Lorentzian spaces. In Section 3, we will extend the boost theory in $[8,10]$ to the case of multi-ends. In Section 4, we will set up a perturbation problem for the mean curvature of graphs. We will take initial data sets as parameters and use linear theory in $[2,7,19]$ and the Quantitative Inverse Function Theorem 4.10. Finally, we will prove the main results in Section 4.4 . 


\section{Preliminaries}

In this section, we give some preliminary results on the weighted Sobolev space theory and the geometry of hypersurfaces in Lorentzian spaces.

\subsection{Weighted Sobolev space theory}

Here we give our definition of the weighted Sobolev space. Most of the results here can be found in $[7,8,10]$. We will mainly talk about two types of domains.

\section{Type 1 domain: sub-domain of $\mathbb{R}^{3}$.}

Let $U$ be an open set in $\mathbb{R}^{n}, \sigma(x)=\left(1+|x|^{2}\right)^{1 / 2}$ for $x \in \mathbb{R}^{n}$, and $V$ a finite dimensional vector space. Given $s \in \mathbb{N}, \delta \in \mathbb{R}$.

Definition 2.1. $C_{\delta}^{s}(U)$ is the Banach space of $C^{s}$ functions $u: U \rightarrow V$, with finite norm

$$
\|u\|_{C_{\delta}^{s}(U)}=\sup _{U}\left\{\sum_{|\alpha| \leq s} \sigma^{\delta+|\alpha|}\left|D^{\alpha} u\right|\right\} .
$$

$H_{s, \delta}(U)$ is the class of functions $u: U \rightarrow V$, with weak derivatives up to order $s$, such that $\sigma^{\delta+|\alpha|} D^{\alpha} u \in L^{2}(U)$ for all $|\alpha| \leq s . H_{s, \delta}(U)$ is a Hilbert space with inner product:

$$
\left\langle u_{1}, u_{2}\right\rangle_{H_{s, \delta}(U)}=\sum_{|\alpha| \leq s}\left\langle\sigma^{\delta+|\alpha|} D^{\alpha} u_{1}, \sigma^{\delta+|\alpha|} D^{\alpha} u_{2}\right\rangle_{L^{2}(U)}
$$

Then the norm is: $\|u\|_{H_{s, \delta}(U)}=\langle u, u\rangle_{H_{s, \delta}(U)}^{1 / 2}$.

Now we will list some properties of $H_{s, \delta}(U)$, which can be found in $[7$, $8,10]$. We will use the following notion on the geometry of domains. Given $0<\epsilon \leq 1$, and $\phi_{\epsilon}: \mathbb{R}^{3} \rightarrow \mathbb{R}^{3}$ defined by $\phi_{\epsilon}(x)=\frac{x}{(\sigma(x))^{1-\epsilon}}$. An open subset $U \subset \mathbb{R}^{3}$ is said to have the extended cone property if $\phi_{\epsilon}(U)$ has the cone property $^{3}$ for each $0<\epsilon \leq 1$.

Lemma 2.2. Given $U$ satisfying the extended cone property,

(i) (embedding). If $s^{\prime}<s-\frac{n}{2}$ and $\delta^{\prime}<\delta+\frac{n}{2}$, the inclusion $H_{s, \delta}(U) \subset$ $C_{\delta^{\prime}}^{s^{\prime}}(U)$ is continuous;

\footnotetext{
${ }^{3}$ See the remark under [10, Definition 2.3].
} 
(ii) (multiplication). If $s \leq s_{1}, s_{2}, s<s_{1}+s_{2}-\frac{n}{2}$ and $\delta<\delta_{1}+\delta_{2}+\frac{n}{2}$, the multiplication $\left(f_{1}, f_{2}\right) \rightarrow f_{1} f_{2}$ is continuous: $H_{s_{1}, \delta_{1}}(U) \times H_{s_{2}, \delta_{2}}(U) \rightarrow$ $H_{s, \delta}(U)$.

Hence $H_{s, \delta}(U)$ is a Banach algebra if $s>\frac{n}{2}$ and $\delta>-\frac{n}{2}$.

\section{Type 2 domain: manifold which is Euclidean at infinity.}

Let $(\Sigma, e)$ be an $n$-dimensional manifold which is Euclidean at infinity. Let $x=\left\{x^{i}\right\}$ be the local coordinates, where $\left\{x^{i}\right\}$ is the pull back of the standard coordinates on $\mathbb{R}^{n} \backslash B_{R}$ when restricted to $E_{i}$, and $e=d s_{0}^{2}=$ $\sum_{i=1}^{n}\left(d x^{i}\right)^{2}$ on $E_{i}$. Fix a point $O \in \Sigma_{\text {int }}$, and define a function on $\Sigma$ by

$$
\sigma_{e}(x)=\left(1+d_{e}^{2}(x, O)\right)^{1 / 2}
$$

Clearly $\sigma_{e}(x)$ is equivalent to $\sigma(x)=\left(1+|x|^{2}\right)^{1 / 2}$ on each end $E_{i}$.

When we use $\Sigma$ to model an initial data set, the spacetime should have topology as a sub-domain of $\Sigma \times \mathbb{R}$. Using coordinates $\left(x^{i}, t\right)$ on $\Sigma \times \mathbb{R}$, it has a natural reference metric

$$
\tilde{e}=d t^{2}+e .
$$

For $\theta \in(0,1]$, the boost region $\Omega_{\theta}$ is defined as,

$$
\Omega_{\theta}=\left\{(x, t) \in \Sigma \times \mathbb{R}:|t| \leq \theta \sigma_{e}(x)\right\}
$$

On $\Omega_{\theta}$, the distance function $d_{\tilde{e}}(\cdot, O)$ is equivalent to $d_{e}(\cdot, O)$, so we can use $\sigma_{e}$ to define the weighted Sobolev space on $\Omega_{\theta}$. Given a smooth tensor bundle $E \rightarrow \Sigma$ or $E \rightarrow \Omega_{\theta}$ and $s \in \mathbb{N}, \delta \in \mathbb{R}$.

Definition 2.3. $C_{\delta}^{s}(\Sigma)$ or $C_{\delta}^{s}\left(\Omega_{\theta}\right)$ is the Banach space of $C^{s}$ sections $u$ : $\Sigma \rightarrow E$, or $u: \Omega_{\theta} \rightarrow E$, with finite norm

$$
\|u\|_{C_{\delta}^{s}\left(\Sigma\left(\text { or } \Omega_{\theta}\right)\right)}=\sup _{\Sigma\left(\text { or } \Omega_{\theta}\right)}\left\{\sum_{|\alpha| \leq s} \sigma_{e}^{\delta+|\alpha|}\left|D^{\alpha} u\right|_{e(\text { or } \tilde{e})}\right\} .
$$

$H_{s, \delta}(\Sigma)$ or $H_{s, \delta}\left(\Omega_{\theta}\right)$ is the class of sections $u: \Sigma \rightarrow E$, or $u: \Omega_{\theta} \rightarrow E$ with weak derivatives up to order $s$, such that $\sigma_{e}^{\delta+|\alpha|} D^{\alpha} u \in L^{2}(\Sigma, e)\left(\right.$ or $\left.L^{2}\left(\Omega_{\theta}, \tilde{e}\right)\right)$ 
for all $|\alpha| \leq s . H_{s, \delta}(\Sigma)$ or $H_{s, \delta}\left(\Omega_{\theta}\right)$ is a Hilbert space with inner product:

$$
\begin{aligned}
& \left\langle u_{1}, u_{2}\right\rangle_{H_{s, \delta}(\Sigma)\left(\text { or } H_{s, \delta}\left(\Omega_{\theta}\right)\right)} \\
& \quad=\sum_{|\alpha| \leq s}\left\langle\sigma_{e}^{\delta+|\alpha|} D^{\alpha} u_{1}, \sigma_{e}^{\delta+|\alpha|} D^{\alpha} u_{2}\right\rangle_{L^{2}(\Sigma, e)\left(\text { or } L^{2}\left(\Omega_{\theta}, \tilde{e}\right)\right)}
\end{aligned}
$$

Then the norm is: $\|u\|_{H_{s, \delta}(\Sigma)\left(\text { or } H_{s, \delta}\left(\Omega_{\theta}\right)\right)}=\langle u, u\rangle_{H_{s, \delta}(\Sigma)\left(\text { or } H_{s, \delta}\left(\Omega_{\theta}\right)\right)}^{1 / 2}$.

Remark 2.4. In fact, the definitions are independent of the choice of $e$ on $\Sigma_{\text {int }}$.

Lemma 2.5 ([7], Lemmas 2.4, 2.5, [6], Appendix 1).

(i) (embedding). If $s^{\prime}<s-\frac{n}{2}, \delta^{\prime}<\delta+\frac{n}{2}$, the inclusion $H_{s, \delta}(\Sigma) \subset C_{\delta^{\prime}}^{s^{\prime}}(\Sigma)$ is continuous;

(ii) (multiplication). If $s \leq s_{1}, s_{2}, s<s_{1}+s_{2}-\frac{n}{2}, \delta<\delta_{1}+\delta_{2}+\frac{n}{2}$, the multiplication $\left(f_{1}, f_{2}\right) \rightarrow f_{1} f_{2}$ is a continuous map: $H_{s_{1}, \delta_{1}}(\Sigma) \times H_{s_{2}, \delta_{2}}$ $(\Sigma) \rightarrow H_{s, \delta}(\Sigma)$, hence $H_{s, \delta}(\Sigma)$ is a Banach algebra if $s>\frac{n}{2}, \delta>-\frac{n}{2}$. Furthermore,

$$
\left\|f_{1} f_{2}\right\|_{H_{s, \delta}(\Sigma)} \leq C\left\|f_{1}\right\|_{H_{s_{1}, \delta_{1}}(\Sigma)}\left\|f_{2}\right\|_{H_{s_{2}, \delta_{2}}(\Sigma)}
$$

where $C$ is a constant depending only on $\left\{n, s_{1}, s_{2}, \delta_{1}, \delta_{2}\right\}$.

Divide $\Omega_{\theta}$ as $\Omega_{\theta}=\left(\Omega_{\theta}\right)_{\text {int }} \cup_{i=1}^{l}\left(\Omega_{\theta}\right)_{i}$, where $\left(\Omega_{\theta}\right)_{i}=\left\{(x, t) \in \Omega_{\theta}: x \in\right.$ $\left.E_{i}\right\}$, and $\left(\Omega_{\theta}\right)_{\text {int }}$ the complement. Now $\left(\Omega_{\theta}\right)_{\text {int }}$ is a compact manifold, and $\left(\Omega_{\theta}\right)_{i} \subset \mathbb{R}^{n+1}$ satisfies the extended cone property in the above section, hence Lemma 2.2. By working separately on $\left(\Omega_{\theta}\right)_{i}$ and $\left(\Omega_{\theta}\right)_{\text {int }}$ as in [7] using Lemma 2.2, we have similar results.

Lemma 2.6. $\quad(i)$ (embedding). If $s^{\prime}<s-\frac{n+1}{2}, \delta^{\prime}<\delta+\frac{n+1}{2}$, the inclusion is $H_{s+1, \delta}\left(\Omega_{\theta}\right) \subset C_{\delta^{\prime}}^{s^{\prime}}\left(\Omega_{\theta}\right)$ is continuous;

(ii) (multiplication). If $s \leq s_{1}, s_{2}, s<s_{1}+s_{2}-\frac{n+1}{2}, \delta<\delta_{1}+\delta_{2}+\frac{n+1}{2}$, then the multiplication $\left(f_{1}, f_{2}\right) \rightarrow f_{1} f_{2}$ is a continuous map: $H_{s_{1}, \delta_{1}}$ $\left(\Omega_{\theta}\right) \times H_{s_{2}, \delta_{2}}\left(\Omega_{\theta}\right) \rightarrow H_{s, \delta}\left(\Omega_{\theta}\right)$, hence $H_{s, \delta}\left(\Omega_{\theta}\right)$ is a Banach algebra if $s>\frac{n+1}{2}, \delta>-\frac{n+1}{2}$.

Using ideas similar to the proof of Theorem 2.3 in [10] and Lemma 2.6, we have 
Lemma 2.7 (composition). Given $\Omega_{\theta}, \Omega_{\theta^{\prime}}$ as above and $f: \Omega_{\theta} \rightarrow \Omega_{\theta^{\prime}}$ a differentiable map, such that $|D f|_{\tilde{e}} \geq c>0$ and $f-i d \in H_{s+1, \delta-1}\left(\Omega_{\theta}\right)$ with $s>\frac{n+1}{2}$ and $\delta>-\frac{n+1}{2}$, then for any $s^{\prime} \leq s+1, \delta^{\prime} \in \mathbb{R}$, the composition $u \rightarrow u \circ f$ is an isomorphism as a map:

$$
H_{s^{\prime}, \delta^{\prime}}\left(f\left(\Omega_{\theta}\right)\right) \rightarrow H_{s^{\prime}, \delta^{\prime}}\left(\Omega_{\theta}\right)
$$

Define the function $\tau(x, t)=\frac{t}{\sigma_{e}(x)}$. Denote the level surface of $\tau$ by $\Sigma_{\tau}=$ $\{(x, t) \in \Sigma \times \mathbb{R}: \tau(x, t)=\tau\}$. Then $\Omega_{\theta}$ has a foliation $\Omega_{\theta}=\cup_{\tau \in(-\theta, \theta)} \Sigma_{\tau}$. The restriction norm is defined as:

$$
\|u\|_{H_{s, \delta}\left(\Sigma_{\tau}, \Omega_{\theta}\right)}=\left(\sum_{k=0}^{s}\left\|\left.D_{t}^{k} u\right|_{\Sigma_{\tau}}\right\|_{H_{s-k, \delta+k}(\Sigma)}^{2}\right)^{1 / 2} .
$$

Using ideas similar to the proof of [8, Lemma 3.1], we can get,

Lemma 2.8 (restriction). $\forall \tau \in(-\theta, \theta)$, we have the following continuous inclusion:

$$
H_{s+1, \delta}\left(\Omega_{\theta}\right) \subset H_{s, \delta+\frac{1}{2}}\left(\Sigma_{\tau}, \Omega_{\theta}\right)
$$

for every $s \in \mathbb{N}$ and $\delta \in \mathbb{R}$.

\subsection{Geometry of hypersurface in Lorentzian space}

In this section, we will review the geometry of hypersurfaces in a Lorentzian space. We will mainly focus on the mean curvature of the hypersurface. Notation and part of the results here trace back to [1], and all concepts of Lorentzian space can be found in [24]. Let $(\mathcal{V}, \gamma)$ be a $(3+1)$-dimensional Lorentzian space, with $\langle\cdot, \cdot\rangle$ the metric pairing and $\nabla$ the connection. A smooth function $t \in C^{\infty}(\mathcal{V})$ is called a time function if $\nabla t$ is non-zero, and everywhere timelike, i.e., $\langle\nabla t, \nabla t\rangle<0$. We call a hypersurface $\Sigma$ spacelike if the restriction of $\gamma$ to $\Sigma$ is Riemannian. In a local coordinate system $\left\{x^{i}, t\right\}$, where $t$ is a time function, the metric can be written as (see [1, Equation $(2.12)])$ :

$$
\gamma=-\left(\alpha^{2}-\beta^{2}\right) d t^{2}+2 \beta_{i} d x^{i} d t+g_{i j} d x^{i} d x^{j}
$$

where $\alpha$ is the lapse function, i.e., $\alpha^{2}=-\langle\nabla t, \nabla t\rangle, g_{i j}$ a Riemannian metric, and $\beta=g^{i j} \beta_{i} \partial_{j}$ the shift vector ${ }^{4}$. Here we use $\partial_{t}=\frac{\partial}{\partial t}$ and $\partial_{i}=\frac{\partial}{\partial x^{i}}$ as

\footnotetext{
${ }^{4}$ See [24, Chap 10] for details.
} 
coordinate vectors. The inverse metric $\gamma^{-1}$ is given by:

$$
\gamma^{\mu \nu}=\left[\begin{array}{cc}
-\frac{1}{\alpha^{2}} & \frac{\beta^{i}}{\alpha^{2}} \\
\frac{\beta^{j}}{\alpha^{2}} & g^{i j}-\frac{1}{\alpha^{2}} \beta^{i} \beta^{j}
\end{array}\right],
$$

under coordinate system $\left\{t, x^{1}, x^{2}, x^{3}\right\}$.

We will denote the level surface of the time function $t$ by $\Sigma_{t}=\{p \in$ $\mathcal{V}: t(p)=t\}$. Let $D$ be the gradient operator on $\Sigma_{t}$, and $d i v^{0}$ the divergence operator on $\Sigma_{t}$. The future-directed timelike unit normal $T$ of $\Sigma_{t}$ is given $\mathrm{by}^{5}$ :

$$
T=-\alpha \nabla t=\alpha^{-1}\left(\partial_{t}-\beta\right)
$$

and the second fundamental form $k_{i j}^{0}$ and the mean curvature $H^{0}$ of the slice $\Sigma_{t}$ are given by,

$$
\begin{gathered}
k_{i j}^{0}=\left\langle\partial_{i}, \nabla_{\partial j} T\right\rangle=\frac{1}{2} \alpha^{-1} \partial_{t} g_{i j}-\frac{1}{2} \alpha^{-1} \mathcal{L}_{\beta} g_{i j}, \\
H^{0}=g^{i j} A_{i j}^{0}=\frac{1}{2} \alpha^{-1} g^{i j} \partial_{t} g_{i j}-\alpha^{-1} \operatorname{div}^{0}(\beta) .
\end{gathered}
$$

Given a spacelike hypersurface $\Sigma$, we can always choose local coordinates $\left\{x^{i}, t\right\}$, such that $\Sigma$ is locally the $t=0$ level surface $\Sigma_{0}$. Given a smooth function $u \in C^{\infty}(\Sigma)$, we can study the graph of $u$, i.e., $\operatorname{Graph}_{u}=\left\{\left(x^{i}, u(x)\right)\right\}$ in local coordinates. So we call this $u$ the height function. By extending $u$ parallel to $\mathcal{V}$ requiring that

$$
\partial_{t} u=0,
$$

Graph $_{u}$ can be viewed as level surface of $(u-t)=0$. The unit normal of Graph $_{u}$ is ${ }^{6}$ :

$$
N=\nu(U+T)
$$

where

$$
U=\frac{\alpha D u}{1+\langle\beta, D u\rangle}, \quad \text { and } \quad \nu=\frac{1}{\left(1-|U|_{g}^{2}\right)^{1 / 2}}
$$

\footnotetext{
${ }^{5}$ See Appendix A for details.

${ }^{6}$ See Appendix A for details.
} 
So $\mathrm{Graph}_{u}$ is spacelike if and only if $1-|U|_{g}^{2}>0$, i.e., $\nu$ is well-defined. Define the canonical graphical diffeomorphism $F: \Sigma \rightarrow G r a p h_{u}$ by $F(x)=$ $(x, u(t))$. Then $\operatorname{Graph}_{u}$ has a local coordinate system $\left\{x^{i}: i=1,2,3\right\}$. The coordinate vector frame $\left\{\partial_{i}\right\}$ on $\Sigma$ is passed by $F$ to a local frame

$$
\alpha_{i}=\partial_{i}+u_{i} \partial_{t}: i=1,2,3,
$$

on $\operatorname{Graph}_{u}$. Now denote $M=G r a p h_{u}$. Using this local coordinates, the restriction $\left.\gamma\right|_{M}$ of $\gamma$ to $\operatorname{Graph}_{u}$, denoting by $g_{M}=\left(g_{M}\right)_{i j} d x^{i} d x^{j}$, is given by

$$
\left(g_{M}\right)_{i j}=g_{i j}+\beta_{i} u_{j}+u_{i} \beta_{j}-\left(\alpha^{2}-\beta^{2}\right) u_{i} u_{j} .
$$

Then the inverse metric matrix is calculated in Appendix A by Equation (A.12) as:

$$
\begin{aligned}
\left(g_{M}\right)^{i j} & =g^{i j}-\frac{1}{\alpha^{2}} \beta^{i} \beta^{j}+\frac{\nu^{2}}{\alpha^{2}}(\beta-\alpha U)^{i}(\beta-\alpha U)^{j} \\
& =\gamma^{i j}+\frac{\nu^{2}}{\alpha^{2}}(\beta-\alpha U)^{i}(\beta-\alpha U)^{j} .
\end{aligned}
$$

So the mean curvature $H_{u}$ of the graph $M$ is given by

$$
H_{u}=\left(g_{M}\right)^{i j}\left\langle\nabla_{\alpha_{i}} N, \alpha_{j}\right\rangle_{\gamma} .
$$

\section{Boost evolution}

Fix a 3-manifold $(\Sigma, e)$, which is Euclidean at infinity. Let $\tilde{e}=d t^{2}+e$ be the reference metric (2.1) on $\Sigma \times \mathbb{R}$. Given an integer $s \geq 4$, and a real number $\delta>-2$, we consider vacuum constraint initial data sets $(\Sigma, g, k)$, such that $(g, k) \in \mathcal{V} \mathcal{C}_{s, \delta+\frac{1}{2}}(\Sigma)$. Here boost evolution means that in the spacetime $(\mathcal{V}, \gamma)$ which is evolved by (EVE) taking $(\Sigma, g, k)$ as initial data set, where $\mathcal{V} \subset \Sigma \times$ $\mathbb{R}$, both the future and past temporal distance $\chi_{ \pm}(x)^{7}$ to the boundary of $\mathcal{V}$ is proportional to the space distance $\sigma_{e}(x)$ for $x \in \Sigma$, i.e., $\chi_{ \pm}(x) \geq c \sigma_{e}(x)$ for $c>0$. We will extend the boost evolution on $\mathbb{R}^{3}$ in [10] to the case of $\Sigma$.

\subsection{Reduced Einstein equation and results on compact domain}

Let us review the reduction using harmonic gauge initially introduced by Y. Choquet-Bruhat (see [6]). Using $\left\{x^{i}: i=1,2,3\right\}$ as local coordinates on

\footnotetext{
${ }^{7}$ See $[10]$ for reference.
} 
$\Sigma$, and $x^{\mu}=\left(x^{0}, x^{i}\right)$, with $x^{0}=t$ as coordinates on $\mathcal{V} \subset \Sigma \times \mathbb{R}$, the Ricci curvature can be expressed $\mathrm{as}^{8}$ :

$$
R i c^{\mu \nu}=R_{h}^{\mu \nu}+\frac{1}{2}\left(\gamma^{\mu \alpha} D_{\alpha} \Gamma^{\nu}+\gamma^{\nu \alpha} D_{\alpha} \Gamma^{\mu}\right)
$$

where $\Gamma_{\alpha \beta}^{\mu}$ is the Christoffel symbol of $\gamma, \Gamma^{\mu}=\gamma^{\alpha \beta} \Gamma_{\alpha \beta}^{\mu}$, and

$$
R_{h}^{\mu \nu}=\frac{1}{2}\left\{\gamma^{\alpha \beta} D_{\alpha} D_{\beta} \gamma^{\mu \nu}-B^{\mu \nu}(\gamma, D \gamma)\right\}
$$

with $B^{\mu \nu}=P_{\alpha \beta, \kappa \lambda}^{\mu \nu, \rho \sigma} D_{\rho} \gamma^{\alpha \beta} D_{\sigma} \gamma^{\kappa \lambda}$, and $P$ is a rational function of $\gamma^{\alpha \beta}$. In fact, the EVE $R i c_{\gamma}=0$ is a degenerated differential equation system due to its invariance under diffeomorphic transformations. Harmonic gauge is used to fix this gauge freedom by Y. Choquet-Bruhat, which means that we can choose $i d:(\mathcal{V}, \gamma) \rightarrow(\mathcal{V}, \tilde{e})$ to be a wave map, i.e., $\square_{(\gamma, e)} i d=0^{9}$. Denote

$$
f^{\mu}=\Gamma^{\mu}-\gamma^{\alpha \beta} \tilde{\Gamma}_{\alpha \beta}^{\mu}
$$

to be the harmonic gauge vector, where $\tilde{\Gamma}_{\alpha \beta}^{\mu}$ is the Christoffel symbol of $\tilde{e}$. $f^{\mu}$ is the difference of two connections, hence a tensor, then the harmonic gauge condition reduces to $f^{\mu}=0$, or:

$$
\square_{\gamma} x^{\mu}=-\gamma^{\alpha \beta} \tilde{\Gamma}_{\alpha \beta}^{\mu},
$$

where $\square_{\gamma}$ is the Laplacian operator of the Lorentzian metric $\gamma$, and $\square_{\gamma} x^{\mu}=$ $-\Gamma^{\mu}$. Now under harmonic gauge (3.2), the (EVE) (1.3) is reduced to ${ }^{10}$

$$
\gamma^{\alpha \beta} D_{\alpha} D_{\beta} \gamma^{\mu \nu}=B^{\mu \nu}(\gamma, D \gamma)+\frac{1}{2} \gamma^{\alpha \beta}\left\{\gamma^{\mu \rho} \tilde{R}_{\beta \alpha \rho}^{\nu}+\gamma^{\nu \rho} \tilde{R}_{\beta \alpha \rho}^{\mu}\right\}
$$

where $\tilde{R}$ is the curvature of $\tilde{e}$. The Cauchy data for these equations consist of:

$$
\left.\gamma\right|_{\Sigma}=\phi,\left.\quad D_{t} \gamma\right|_{\Sigma}=\psi
$$

For given initial data set $(g, k)$, we need to construct Cauchy data $(\phi, \psi)$ by requiring $\left.f^{\mu}\right|_{\Sigma}=\left.\left(\Gamma^{\mu}-\gamma^{\alpha \beta} \tilde{\Gamma}_{\alpha \beta}^{\mu}\right)\right|_{\Sigma}=0$. To fix the freedom in choosing a

${ }^{8}$ See $[10$, Section 4$]$ and [24, Section 10.2].

${ }^{9}$ See $[6$, Chapter 6, Section 7.4].

${ }^{10}$ See [6, page 163]. 
harmonic gauge, we require the coordinate system of $\mathcal{V}$ is Gaussian on $\Sigma$, which means:

$$
\phi^{00}=-1, \quad \phi^{0 i}=0, \quad \phi^{i j}=g^{i j} .
$$

The condition $\left.\left(\Gamma^{\mu}-\gamma^{\alpha \beta} \tilde{\Gamma}_{\alpha \beta}^{\mu}\right)\right|_{\Sigma}=0$ requires $^{11}$ :

$$
\psi^{00}=-4 t r_{g} k, \psi^{0 i}=-\left(\Gamma_{g}^{i}-g^{k j} \tilde{\Gamma}_{k j}^{i}\right), \quad \psi^{i j}=2 g^{i k} g^{j l} k_{k l} .
$$

Define a reference Lorentzian metric by

$$
\tilde{\eta}=-d t^{2}+e
$$

When the initial data $(g-e, k) \in H_{s, \delta+\frac{1}{2}}(\Sigma) \times H_{s-1, \delta+\frac{3}{2}}(\Sigma)$, the Cauchy data (3.5) and (3.6) satisfy $(\phi-\tilde{\eta}, \psi) \in H_{s, \delta+\frac{1}{2}}(\Sigma) \times H_{s-1, \delta+\frac{3}{2}}(\Sigma)$. In fact, by the multiplication Lemma $2.5,(g-e, k) \stackrel{\rightarrow}{\rightarrow}(\phi-\tilde{\eta}, \psi)$ is a continuous $\operatorname{map} H_{s, \delta+\frac{1}{2}}(\Sigma) \times H_{s-1, \delta+\frac{3}{2}}(\Sigma) \rightarrow H_{s, \delta+\frac{1}{2}}(\Sigma) \times H_{s-1, \delta+\frac{3}{2}}(\Sigma)$.

To solve (EVE) (1.3), we can first solve the reduced Equation (3.3) by quasilinear theory (see [6, Appendix 3] and [10, Section 5]), and then show that the harmonic gauge is preserved. In fact, Bianchi identity and the reduced equation (3.3) imply that the harmonic gauge vector $f^{\mu}$ satisfies a linear equation ${ }^{12}$ :

$$
\square_{\gamma} f^{\mu}+A(\gamma, D \gamma) D f=0
$$

So we can use uniqueness of linear equations to show that $f^{\mu} \equiv 0$ since we chose $\left.f^{\mu}\right|_{\Sigma}=0$, and the VCEs (1.4) impliy that $\left.\partial_{t} f^{\mu}\right|_{\Sigma}=0^{13}$.

Now we summarize a local version of the existence and causal uniqueness theorem based on the interior region $\Sigma_{\text {int }}$ of $(\Sigma, e)$, which has dimension $n=3$. We can extend the interior region $\Sigma_{\text {int }}$ to contain the annuli $B_{2 R} \backslash B_{R}$ of each end $E_{i}$ of $(\Sigma, e)$. Now define a causal set $\left(V_{\text {int }}\right)_{\theta, \lambda}$ based on $\Sigma_{\text {int }}$ as follows:

$$
\left(V_{\text {int }}\right)_{\theta, \lambda}=\left\{(x, t) \in \Sigma_{\text {int }} \times[-\theta, \theta]:|x| \leq 2 R-\lambda|t|, \text { if } x \in E_{i}\right\},
$$

where $\theta \in(0,1]$ and $\lambda \geq 2$ is a positive number. Now $\left(V_{\text {int }}\right)_{\theta, \lambda}$ has a lateral boundary $L_{\theta, \lambda}=\left\{(x, t) \in\left(V_{\text {int }}\right)_{\theta, \lambda}:|x|=2 R-\lambda|t|\right\}$. When $\lambda$ is large

\footnotetext{
${ }^{11}$ See [6, page 164].

${ }^{12}$ See $[6$, page 167$]$ and $[10$, Section 4].

${ }^{13}$ See [6, page 167$]$.
} 
enough depending only on $e, L_{\theta, \lambda}^{+}=L_{\theta, \lambda} \cap\{t \geq 0\}$ (or $L_{\theta, \lambda}^{-}=L_{\theta, \lambda} \cap\{t \leq 0\}$ ) is spacelike and ingoing (or outgoing) w.r.t. $\tilde{\eta}$, hence $\left(\left(V_{\text {int }}\right)_{\theta, \lambda}, \tilde{\eta}\right)$ is causal ${ }^{14}$.

Combining Theorem 7.4, Theorem 8.3 of Chap 6, and Corollary 4.8, Theorem 4.11, Theorem 4.13 of Appendix 3 in [6], and using a cutoff argument as in Theorem 3.7, we have the following well-known local existence and uniqueness theorem,

Theorem 3.1. Given an integer $s \geq 4$. For a vacuum constraint data set $\left(\Sigma_{\text {int }}, g, k\right)$, with $(g-e, k) \in H_{s}\left(\Sigma_{\text {int }}\right) \times H_{s-1}\left(\Sigma_{\text {int }}\right)$, and $g \geq \lambda_{0} e$ for some $\lambda_{0}>0$, there exists $\theta>0, \lambda \geq 2$ and $C_{0}>0$ depending only on $\lambda_{0}$ and $\|g-e\|_{H_{s}\left(\Sigma_{\mathrm{int}}\right)}+\|k\|_{H_{s-1}\left(\Sigma_{\mathrm{int}}\right)}$, and a unique regularly sliced ${ }^{15}$ Lorentzian metric $\gamma$ solving the reduced $E V E$ (3.3) on $\left(V_{\mathrm{int}}\right)_{\theta, \lambda}$, taking (3.5) and (3.6) as initial value which is given by $(g, k)$, such that $(\gamma-\tilde{\eta}) \in H_{s}\left(\left(V_{\text {int }}\right)_{\theta, \lambda}\right)$, with

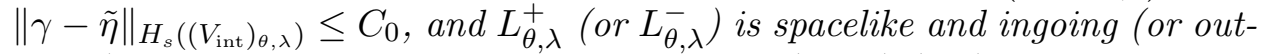
going) w.r.t $\gamma$. Furthermore, $\gamma$ is a solution of (EVE) (1.3) under harmonic gauge.

\subsection{Boost evolution on manifold Euclidean at infinity}

We first modify the linear boost theory in [10] to the case based on an Euclidean end $E \cong \mathbb{R}^{n-1} \backslash B_{R}$. Let us fix a special type of boost regions. Denote $\bar{x}=\left(x^{1}, \ldots, x^{n-1}\right) \in \mathbb{R}^{n-1}$, such that $x=(\bar{x}, t) \in \mathbb{R}^{n}$. Later on, we will denote the index for $t$-coordinates as 0 , while index for $\bar{x}$ as $i$ with $i=1, \ldots, n-1$. Let $\bar{\sigma}(\bar{x})=\left(1+|\bar{x}|^{2}\right)^{1 / 2}$. For $\theta \in(0,1 / 2], \lambda \geq 2$ and a given end $E \cong \mathbb{R}^{n-1} \backslash B_{R}$, the boost region $V_{\theta, \lambda}$ based on $E$ is defined as:

$$
V_{\theta, \lambda}=\left\{(\bar{x}, t) \in \mathbb{R}^{n}, \frac{|t|}{\bar{\sigma}(\bar{x})}<\theta,|\bar{x}| \geq R+\lambda|t|\right\} .
$$

Define the function $\tau$ as $\tau(x)=\frac{t}{\bar{\sigma}(\bar{x})}$. Then the level surface of $\tau$ is $E_{\tau}=$ $\left\{x \in V_{\theta, \lambda}: \tau(x) \equiv \tau\right\} . V_{\theta, \lambda}$ has a foliation:

$$
V_{\theta, \lambda}=\cup_{\tau \in(-\theta, \theta)} E_{\tau}
$$

The lateral boundary of $V_{\theta, \lambda}$ is defined as,

$$
L_{\theta, \lambda}=\left\{(\bar{x}, t) \in V_{\theta, \lambda}:|\bar{x}|=R+\lambda|t|\right\} .
$$

Denote the upper part of $V_{\theta, \lambda}$ by $V_{\theta, \lambda}^{+}=\left\{(\bar{x}, t) \in V_{\theta, \lambda}: t \geq 0\right\}$, then the boundary $\partial V_{\theta, \lambda}^{+}$is constituted by $E, E_{\theta}$ and the upper lateral boundary

\footnotetext{
${ }^{14}$ See [6, Appendix 3, Definition 2.11].

${ }^{15}$ See $[6$, page 397 and page 585$]$.
} 
$L_{\theta, \lambda}^{+}=L_{\theta, \lambda} \cap V_{\theta, \lambda}^{+}$. Similarly, we have $V_{\theta, \lambda}^{-}=\left\{(\bar{x}, t) \in V_{\theta, \lambda}: t \leq 0\right\}$ and the lower lateral boundary $L_{\theta, \lambda}^{-}=L_{\theta, \lambda} \cap V_{\theta, \lambda}^{-}$. Clearly $V_{\theta, \lambda}^{ \pm}$and the slices $E_{\tau}$ satisfy the extended cone property in $\mathbb{R}^{n}$ and $\mathbb{R}^{n-1}$, respectively as in Section 2.1, and hence satisfy Lemma 2.2.

We introduce a class of hyperbolic metrics on $V_{\theta, \lambda}$ using the foliation $\left\{E_{\tau}\right\}_{\tau \in(-\theta, \theta)}$. The function $\tau$ is in fact a time function on $\left(V_{\theta, \lambda}, \eta\right)$, where $\eta=-d t^{2}+\sum_{i=1}^{n-1}\left(d x^{i}\right)^{2}$ is the Minkowski metric. Let $\tilde{n}_{\mu}$ be the unit future co-normal of the foliation $\left\{E_{\tau}: \tau \in(-\theta, \theta)\right\}$, given by

$$
\tilde{n}=\tilde{N} D \tau=\frac{1}{\sqrt{1-\tau^{2}|\bar{x}|^{2} \sigma^{-2}(\bar{x})}}\left(d t-\frac{\tau}{\sigma(\bar{x})} x^{i} d x^{i}\right),
$$

where $\tilde{N}$ is the lapse function for the foliation $\left\{E_{\tau}\right\}$, defined by: $\tilde{N}^{-2}=$ $-\langle D \tau, D \tau\rangle_{\eta}=\frac{1-\tau^{2}|\bar{x}|^{2} \sigma^{-2}(\bar{x})}{\sigma^{2}(\bar{x})} . \tilde{n}$ can be viewed as a standard calibration for the foliation $V_{\theta, \lambda}=\cup E_{\tau}$, which is used to define the "regularity" of hyperbolicity. Denoting $|\cdot|$ as the standard Euclidean norm for tensors on $V_{\theta}$, we have ${ }^{16}$ :

Definition 3.2. A $C^{0}$ covariant symmetric 2-tensor $\gamma^{\mu \nu}$ on $V_{\theta, \lambda}$ is called regularly hyperbolic, if there exist positive numbers $a, b$, and $C$ such that in $V_{\theta, \lambda}$ :

(1) $-\gamma^{\mu \nu} \tilde{n}_{\mu} \tilde{n}_{\nu} \geq a$;

(2) for all tangent covectors $\zeta_{\mu}$ of $E_{\tau}$, i.e., $\gamma^{\mu \nu} \zeta_{\mu} \tilde{n}_{\nu}=0$, we have $\gamma^{\mu \nu} \zeta_{\mu} \zeta_{\nu} \geq$ $b|\zeta|^{2}$

(3) $|\gamma| \leq C$;

(4) The upper (or lower) lateral boundary $L_{\theta, \lambda}^{+}\left(\right.$or $\left.L_{\theta, \lambda}^{-}\right)$is spacelike and ingoing (or out going) w.r.t. $\gamma$, i.e., every timelike curve entering $V_{\theta, \lambda}^{+}$ (or every timelike curve exiting $V_{\theta, \lambda}^{-}$) is past directed.

The coefficient of regular hyperbolicity of $\gamma$ is defined as,

$$
h=\max \left\{\frac{1}{a}, \frac{1}{b}, C\right\} .
$$

Remark 3.3. Condition (4) implies that this type of $V_{\theta, \lambda}$ is a causal subset based on $E$ w.r.t. $\gamma^{17}$. Here we briefly talk about the criterion for Condition (4) to be true. We mainly discuss the case $L_{\theta, \lambda}^{+}$, and $L_{\theta, \lambda}^{-}$is similar. The defining function of $L^{+}$is given by $l(\bar{x}, t)=\lambda t+R-|\bar{x}|$, so the normal co-vector of $L^{+}$is given by $d l=\lambda d t-d \bar{r}$, where $\bar{r}=|\bar{x}|$. Now $d l=$

\footnotetext{
${ }^{16}$ See also [10, Definition 4.1].

${ }^{17}$ See Definition 2.11 of [6, Appendix 3].
} 
$\lambda\left(d t-\frac{\tau \bar{r}}{\sigma(\bar{x})} d \bar{r}\right)+\left(\lambda \frac{\tau \bar{r}}{\sigma(\bar{x})}-1\right) d \bar{r}=\lambda \sqrt{1-\tau^{2}|\bar{x}|^{2} \sigma^{-2}(\bar{x})} \tilde{n}+\left(\lambda \frac{\tau \bar{r}}{\sigma(\bar{x})}-1\right) d \bar{r}$. So using the regularly hyperbolicity, we have $\gamma(d l, d l) \leq \lambda^{2}\left(1-\theta^{2}\right) \gamma(\tilde{n}, \tilde{n})+$ $\lambda(\theta \lambda-1) C \leq-a \lambda^{2}\left(1-\theta^{2}\right)+C \lambda(\theta \lambda-1)<0$, when $\lambda$ is chosen large enough depending only on $a$ and $C$, hence depending only on $h$.

Remark 3.4. The set of regularly hyperbolic metrics on $V_{\theta, \lambda}$ is open in the space $C^{0}\left(V_{\theta, \lambda}\right)$ of bounded continuous covariant symmetric 2 -tensors. In fact, $\eta$ is regular hyperbolic with $a=1, b=1-\theta^{2}$ and $C=\sqrt{n}$, and $L_{\theta, \lambda}$ is space-like and ingoing w.r.t $\eta$ when $\lambda \geq 2$. Since the space-like and ingoing condition for $L_{\theta, \lambda}$ is an open condition, there exists a small $\epsilon>0$, depending only on $\theta, \lambda$ and $n$, such that any $C^{0}$ covariant symmetric 2-tensor $\gamma$, with $|\gamma-\eta| \leq \epsilon$, is regularly hyperbolic in $V_{\theta, \lambda}$.

Now consider a family of linear differential operators of second order in $V_{\theta, \lambda}$ :

$$
L u=\Sigma_{k=0}^{2} a_{k} \cdot D^{k} u
$$

where $u$ and $L u$ are $\mathbb{R}^{N}$-valued functions on $V_{\theta, \lambda}$, and $a_{k}$ are matrix valued functions. The following hypotheses are required for the existence theory:

- Hypothesis (1) (weak coupling and hyperbolicity). $a_{2}=\gamma I d$, i.e., $\left(a_{2}\right)_{J}^{\mu \nu I}=\gamma^{\mu \nu} \delta_{J}^{I}, \mu, \nu=0, \ldots, n-1, I, J=1, \ldots, N$, where $\gamma$ is a regularly hyperbolic metric on $V_{\theta, \lambda}$.

- Hypothesis (2) (regularity). There exist integers $s_{k}$ and real numbers $\delta_{k}$, such that: $s_{k}>\frac{n}{2}+k-1, \delta_{k}>2-k-\frac{n}{2}: 0 \leq k \leq 2$, and (1) $a_{k} \in H_{s_{k}, \delta_{k}}\left(V_{\theta, \lambda}\right)$ for $k=0,1 ;(2) \gamma-\eta \in H_{s_{2}, \delta_{2}}\left(V_{\theta, \lambda}\right)$.

Remark 3.5. Now denote

$$
\begin{aligned}
& s^{\prime}=\min _{0 \leq k \leq 2}\left\{s_{k}\right\}+1 \\
& m=\|\gamma-\eta\|_{H_{s_{2}, \delta_{2}}\left(V_{\theta, \lambda}\right)}+\Sigma_{k=0}^{1}\left\|a_{k}\right\|_{H_{s_{k}, \delta_{k}}\left(V_{\theta, \lambda}\right)}, \\
& \mu=\|\gamma-\eta\|_{H_{s_{2}-1, \delta_{2}+1 / 2}\left(E, V_{\theta, \lambda}\right)}+\sum_{k=0}^{1}\left\|a_{k}\right\|_{H_{s_{k}-1, \delta_{k}+1 / 2}\left(E, V_{\theta, \lambda}\right)} .
\end{aligned}
$$

By the restriction Lemma 2.8, $\mu \leq \mathrm{cm}$. Using the multiplication Lemma 2.2, the regularity hypothesis (2) implies that

$$
L: H_{s+1, \delta}\left(V_{\theta, \lambda}\right) \rightarrow H_{s-1, \delta+2}\left(V_{\theta, \lambda}\right),
$$

is a continuous map for $1 \leq s \leq s^{\prime}$ and $\delta \in \mathbb{R}$. 
Then we have the existence and uniqueness theorem for linear systems.

Theorem 3.6. Let $L$ be a differential operator defined by (3.14) in $V_{\theta, \lambda}$, satisfying Hypotheses (1) and (2). Let $\beta \in H_{s-1, \delta+2}\left(V_{\theta, \lambda}\right), \phi \in H_{s, \delta+\frac{1}{2}}(E)$ and $\psi \in H_{s-1, \delta+\frac{3}{2}}(E)$, with $2 \leq s \leq s^{\prime}, \delta \in \mathbb{R}$. Then the Cauchy problem:

$$
L u=\beta,\left.\quad u\right|_{\Sigma}=\phi,\left.D_{t} u\right|_{\Sigma}=\psi,
$$

has a unique solution $u \in H_{s, \delta}\left(V_{\theta, \lambda}\right)$, and satisfies the estimates:

$$
\|u\|_{H_{s, \delta}\left(V_{\theta, \lambda}\right)} \leq c \theta^{\frac{1}{2}}\left\{\|\phi\|_{H_{s, \delta+\frac{1}{2}}(E)}+\|\psi\|_{H_{s-1, \delta+\frac{3}{2}}(E)}+\|\beta\|_{H_{s-1, \delta+2}\left(V_{\theta, \lambda}\right)}\right\},
$$

where $c$ is a continuous increasing function of $(\theta, h, m)$, and $h, m$ are defined by Equations (3.13) and (3.16), respectively.

Proof. It follows from the energy estimates in Theorem A.8 in Appendix A.2, and similar approximation argument as in the proof of [8, Theorem 5.1] and [10, Theorem 4.1].

Now we extend the existence theory for the boost problem in [10] to $\Sigma$. Let $\Omega_{\theta}$ be the boost region based on $\Sigma$ as defined in (2.2). We will construct a solution to the reduced EVE (3.3) in $\Omega_{\theta}$. We deal with the boost evolution separately on the interior region $\Sigma_{\text {int }}$ and on each end $E_{i}$. On compact set $\Sigma_{\text {int }}$, we can use Theorem 3.1. On each end $E$, we can complete the initial data $\left.(g, k)\right|_{E}$ to $\mathbb{R}^{3}$ and apply the boost theory in [10] to get existence. Then we can cut off the solution in the causal set based on the end $E$ by our linear Theorem 3.6. Causal uniqueness (see [6, Appendix 3, Corollary 4.8]) tells us that the solutions we got based on $\Sigma_{\text {int }}$ and $E_{i}$ 's match together to a global solution.

Theorem 3.7. For $s \geq 4, \delta>-2$. Given vacuum data $(g, k) \in \mathcal{V C}_{s, \delta+\frac{1}{2}}(\Sigma)$, with $g \geq \lambda_{0}$ e for some $\lambda_{0}>0$, there exit $\theta \in(0,1)$ and $C_{0}>0$ depending only on $\lambda_{0},\|g-e\|_{H_{s, \delta+\frac{1}{2}}(\Sigma)}+\|k\|_{H_{s-1, \delta+\frac{3}{2}}(\Sigma)}$, and a unique Lorentzian metric $\gamma$ solving the reduced $E V E$ (3.3) on $\Omega_{\theta}$, which has Cauchy data $(\phi, \psi)$ on $\Sigma$ given by $(g, k)$ in (3.5) (3.6), such that $(\gamma-\tilde{\eta}) \in H_{s, \delta}\left(\Omega_{\theta}\right)$, and $\| \gamma-$ $\tilde{\eta} \|_{H_{s, \delta}\left(\Omega_{\theta}\right)} \leq C_{0}$. Furthermore $\gamma$ is the solution to EVE (1.3) under harmonic gauge.

Proof. We first focus on a fixed end $E$. In fact, we can extend $\left.(g, k)\right|_{E}$ to $(\bar{g}, \bar{k})$ on $\mathbb{R}^{3}$ by a cutting and pasting method, such that $(\bar{g}, \bar{k})=(g, k)$ on $E$ with $\bar{g} \geq \bar{\lambda} \delta$, where $\bar{\lambda} \geq c^{-1} \lambda_{0}$ and $\|\bar{g}-\delta\|_{H_{s, \delta+\frac{1}{2}}\left(\mathbb{R}^{3}\right)}+\|\bar{k}\|_{H_{s-1, \delta+\frac{3}{2}}\left(\mathbb{R}^{3}\right)} \leq$ 
$c\left(\|g-e\|_{H_{s, \delta+\frac{1}{2}}(E)}+\|k\|_{H_{s-1, \delta+\frac{3}{2}}(E)}\right)$ for some fixed $c>1$. By Lemma 5.1 and Theorem 6.1 in [10], there exist $C_{1}>0$ and $\theta_{1} \in(0,1)$ depending only on $\bar{\lambda}$ and $\|\bar{g}-\delta\|_{H_{s, \delta+\frac{1}{2}}}\left(\mathbb{R}^{3}\right)+\|\bar{k}\|_{H_{s-1, \delta+\frac{3}{2}}}\left(\mathbb{R}^{3}\right)$, and a unique solution $\bar{\gamma}$ to the reduce $\operatorname{EVE}(3.3)$ on $\Omega_{\theta_{1}}$, taking on $\mathbb{R}^{3}$ the Cauchy data $(\bar{\phi}, \bar{\psi})$ given by $(\bar{g}, \bar{k})$ as in (3.5) and (3.6) where the Christoffel symbol for $\mathbb{R}^{3}$ is $\left.\tilde{\Gamma}\right|_{\mathbb{R}^{3}}=0$, and $\|\bar{\gamma}-\eta\|_{H_{s, \delta}\left(\Omega_{\theta_{1}}\right)}<C_{1}$. Here $\Omega_{\theta_{1}}$ is the boost region $(2.2)$ when $\Sigma=R^{3}$. Furthermore, $\bar{\gamma}$ is regularly hyperbolic ${ }^{18}$, with the coefficient of regularly hyperbolicity $h_{1}$ depending only on $\bar{\lambda}$ and $\|\bar{g}-\delta\|_{H_{s, \delta+\frac{1}{2}}\left(\mathbb{R}^{3}\right)}+\|\bar{k}\|_{H_{s-1, \delta+\frac{3}{2}}}\left(\mathbb{R}^{3}\right)$.

We claim that there exists a $\lambda_{1}>2$ depending only on $h_{1}$, such that $\bar{\gamma}$ is regularly hyperbolic on $V_{\theta_{1}, \lambda_{1}}$. The first three conditions in Definition 3.2 are naturally satisfied since $\bar{\gamma}$ is regularly hyperbolic in $\Omega_{\theta_{1}}$ (see $[10$, Definition 4.1]). Condition (4) is true if we take take $\lambda_{1}$ large enough depending only on the regularly hyperbolicity $h_{1}$ of $\bar{\gamma}$ as discussed in Remark 3.3.

Then we claim that $\bar{\gamma}$ is a solution of (EVE) (1.3) in harmonic gauge inside the causal set $V_{\theta_{1}, \lambda_{1}}$. In fact, since $(g, k)$ is a solution of (VCE) (1.4) on $E$, the harmonic gauge condition $f^{\mu}=\Gamma_{\bar{\gamma}}^{\mu}=0$ and $\partial_{t} f^{\mu}=0$ on $E$ are satisfied by the choice of initial conditions (3.5) and (3.6). Notice that $f$ satisfies a linear equation (3.8), which satisfies the requirement of Theorem 3.6 by the argument in [10, page 293]. Hence the harmonic gauge vector $f=0$ in $V_{\theta_{1}, \lambda_{1}}$ by the estimate (3.19) in Theorem 3.6, hence $\bar{\gamma}$ is a solution of EVE (1.3) on $V_{\theta_{1}, \lambda_{1}}$.

Now denote the restriction $\bar{\gamma}$ to $V_{\theta_{1}, \lambda_{1}}$ by $\gamma$. We claim that $\left(V_{\theta_{1}, \lambda_{1}}, \gamma\right)$ is uniquely determined by $\left.(g, k)\right|_{E}$ when $\gamma$ is regularly hyperbolic on $V_{\theta_{1}, \lambda_{1}}$. Suppose $\gamma_{1}$ and $\gamma_{2}$ are two such solutions of the reduced EVE (3.3) as above with initial value given by (3.5) and (3.6) from vacuum data $\left(g_{1}, k_{1}\right)$ and $\left(g_{2}, k_{2}\right)$, respectively. Then $\left\|\gamma_{i}-\eta\right\|_{H_{s, \delta}(V)}$ are uniformly bounded by the corresponding norm of $\left(g_{i}-\eta, k_{i}\right)$. Now subtract the reduced EVE (3.3) satisfied by $\gamma_{1}$ and $\gamma_{2}$ :

(3.20)

$\gamma_{1}^{\alpha \beta} D_{\alpha} D_{\beta}\left(\gamma_{1}^{\mu \nu}-\gamma_{2}^{\mu \nu}\right)-\left(D^{2} \gamma_{2}\right)\left(\gamma_{2}-\gamma_{1}\right)-\left(B\left(\gamma_{1}, D \gamma_{1}\right)-B\left(\gamma_{2}, D \gamma_{2}\right)\right)=0$,

where (see [10, Equations (4.4) and (4.5)])

$$
\begin{aligned}
B\left(\gamma_{1}, D \gamma_{1}\right)-B\left(\gamma_{2}, D \gamma_{2}\right)= & P\left(\gamma_{1}\right)\left(D \gamma_{1}\right)^{2}-P\left(\gamma_{2}\right)\left(D \gamma_{2}\right)^{2} \\
= & \left(P\left(\gamma_{1}\right)-P\left(\gamma_{2}\right)\right)\left(D \gamma_{1}\right)^{2} \\
& +P\left(\gamma_{2}\right)\left(D \gamma_{1}+D \gamma_{2}\right)\left(D \gamma_{1}-D \gamma_{2}\right)
\end{aligned}
$$

\footnotetext{
${ }^{18}$ Here regularly hyperbolicity is given in [10, Definition 4.1], which only requires the first three conditions in our Definition 3.2.
} 
Here $P$ is a rational function of $\gamma$. Using the multiplication Lemma 2.2, $\left(D \gamma_{1}\right)^{2}, P\left(\gamma_{2}\right)\left(D \gamma_{1}+D \gamma_{2}\right) \in H_{s-1, \delta+1}(V)$. Using the mean value inequality, and the Sobolev embedding Lemma 2.2, we have the pointwise estimates:

$$
\left|P\left(\gamma_{1}\right)-P\left(\gamma_{2}\right)\right| \leq C\left|\gamma_{1}-\gamma_{2}\right|
$$

where $C$ depends only on $\left\|\gamma_{i}-\eta\right\|_{H_{s, \delta}(V)}, i=1,2$. Now viewing equation (3.20) as a differential equation for $\left(\gamma_{1}-\gamma_{2}\right)$, and using the first energy estimate Lemma A.4 in Appendix A.2, we have

$$
\begin{aligned}
\left\|\gamma_{1}-\gamma_{2}\right\|_{H_{1, \delta+\frac{1}{2}}\left(E_{\tau}, V\right)} & \leq C\left\|\gamma_{1}-\gamma_{2}\right\|_{H_{1, \delta+\frac{1}{2}}(E, V)} \\
& \leq C\left(\left\|g_{1}-g_{2}\right\|_{H_{1, \delta+\frac{1}{2}}(E)}+\left\|k_{1}-k_{2}\right\|_{H_{0, \delta+\frac{3}{2}}(E)}\right) .
\end{aligned}
$$

Hence the uniqueness is true.

Combing all the above, we get a unique regularly hyperbolic solution $\gamma$ to the (EVE) under harmonic gauge in $V_{\theta_{1}, \lambda_{1}}$, where $\theta_{1}, \lambda_{1}$ and $\|\gamma-\eta\|_{H_{s, \delta}(V)} 19$ depend only on $\lambda_{0}$ and $\|g-e\|_{H_{s, \delta+\frac{1}{2}}(E)}+\|k\|_{H_{s-1, \delta+\frac{3}{2}}(E)}$.

Now extend $\Sigma_{\text {int }}$ to include the annuli $B_{r} \backslash B_{R} \subset E_{i}$, and take the solution $\gamma$ inside the causal set $\left(V_{\text {int }}\right)_{\theta_{0}, \lambda_{0}}$ based on $\Sigma_{\text {int }}$ by Theorem 3.1. We can combine it with all the solutions $\left(V_{\theta_{i}, \lambda_{i}}, \gamma\right)$ on each end $E_{i}$. Now causal uniqueness (see [6, Appendix 3, Corollary 4.8]) implies that they coincide in the intersection of $\left(V_{\text {int }}\right)_{\theta_{0}, \lambda_{0}}$ and $V_{\theta_{i}, \lambda_{i}}$, since $\left(V_{\text {int }}\right)_{\theta_{0}, \lambda_{0}} \cap V_{\theta_{i}, \lambda_{i}}$ is a causal set based on $\Sigma_{\text {int }} \cap E$ w.r.t. $\gamma$ by our construction. So by choosing the smallest $\theta$, such that $\Omega_{\theta} \subset\left(V_{\text {int }}\right)_{\theta_{0}, \lambda_{0}} \cup_{i=1}^{l} V_{\theta_{i}, \lambda_{i}}$, we get the conclusion.

\section{Perturbation method}

Here we will apply the Inverse Function Theorem (see [5, 20]) to find maximal graphs in the spacetime evolution of given $\mathrm{AF}$ vacuum data sets with small trace. Fix a 3 -manifold $(\Sigma, e)$ which is Euclidean at infinity. We always assume $s \in \mathbb{N}, s \geq 4$, and $\delta>-2$. Consider the vacuum data sets $(\Sigma, g, k)$, with $(g, k) \in \mathcal{V C}_{s+1, \delta+\frac{1}{2}}(\Sigma)$. Let $(\mathcal{V}, \gamma)$ be the boost evolution of $(g, k)$ given by Theorem 3.7, then we will study the graph of a given function $u$ in the spacetime $(\mathcal{V}, \gamma)$. We will take $(g, k)$ as parameters, and study the perturbation problem for the mean curvature $H_{u}$ of this graph. We will show that for appropriately chosen weighted Sobolev spaces, the linearization of $H_{u}$ with respect to $u$ is invertible in certain sense.

${ }^{19}$ The bound for $(\gamma-\eta)$ also comes directly by Theorem 3.6. 


\subsection{Differentiability of mean curvature operator}

Given a vacuum data set $(g, k) \in \mathcal{V} \mathcal{C}_{s+1, \delta+\frac{1}{2}}(\Sigma)$, with $g \geq \lambda e$ for some $\lambda>0$. By Theorem 3.7, there exists a uniform $\theta \in(0,1)$ and a uniform $C>0$, depending only on $\lambda$ and $\|g-e\|_{H_{s+1, \delta+\frac{1}{2}}(\Sigma)}+\|k\|_{H_{s, \delta+\frac{3}{2}}(\Sigma)}$, and a unique Lorentzian solution $\gamma$ of the reduced $\operatorname{EVE}^{2}(3.3)$ on $\Omega_{\theta}$, taking $(g, k)$ as initial data, and $\|\gamma-\tilde{\eta}\|_{H_{s+1, \delta}\left(\Omega_{\theta}\right)} \leq C$. Moreover, from the proof of Theorem 3.7, the regularly hyperbolic coefficient $h$ of $\gamma$ in each boost end $V_{\theta_{i}, \lambda_{i}}$, and the regularly sliced coefficient ${ }^{20}$ of $\gamma$ in $\left(V_{\text {int }}\right)_{\theta_{0}, \lambda_{0}}$ are all uniformly bounded by a constant depending only on $\lambda$ and the norm of $(g, k)$. Hence the determinant of $\gamma^{\mu \nu}$ is bounded away from 0 by a constant depending only on $\lambda$ and the norm of $(g, k)$.

Now let us summarize some properties of the metric components of $\gamma$.

Lemma 4.1. For $s \geq 3, \delta>-2$. Given a $(3+1)$ Lorentz metric $\gamma^{\mu \nu}$ of form (2.6) in $\Omega_{\theta}$ with $(\gamma-\tilde{\eta})^{\mu \nu} \in H_{s, \delta}\left(\Omega_{\theta}\right)$, if the determinant det $\left(\gamma^{\mu \nu}\right) \leq-\tilde{\lambda}$ for some $\tilde{\lambda}>0$, then $(\gamma-\tilde{\eta})_{\mu \nu}$ lies in $H_{s, \delta}\left(\Omega_{\theta}\right)$, and in the metric form (2.5) and (2.6) of $\gamma$, the components $\left(\alpha^{-2}-1\right),(\alpha-1), \beta^{i}, \beta_{i}, g^{i j}-$ $e^{i j}, g_{i j}-e_{i j}$ all lie in $H_{s, \delta}\left(\Omega_{\theta}\right)$. Furthermore, their norms are all bounded by a constant depending only on $\tilde{\lambda}$ and $\|\gamma-\tilde{\eta}\|_{H_{s, \delta}\left(\Omega_{\theta}\right)}$.

Proof. The inverse matrix $\gamma_{\mu \nu}=\operatorname{det}\left(\gamma^{\mu \nu}\right) \operatorname{adj}\left(\gamma^{\mu \nu}\right)$, where $\operatorname{adj}\left(\gamma^{\mu \nu}\right)$ is the adjoint matrix of $\gamma^{\mu \nu}$. Since $\operatorname{det}\left(\gamma^{\mu \nu}\right)$ is bounded away from 0 by $\tilde{\lambda}$, the Banach algebra property (Lemma 2.6) of $H_{s, \delta}\left(\Omega_{\theta}\right)$ implies that $\gamma_{\mu \nu}-\tilde{\eta}_{\mu \nu}$ also lies in $H_{s, \delta}\left(\Omega_{\theta}\right)$, with $\left\|\gamma_{\mu \nu}-\tilde{\eta}_{\mu \nu}\right\|_{H_{s, \delta}\left(\Omega_{\theta}\right)}$ bounded by a constant depending only on $\tilde{\lambda}$ and $\left\|(\gamma-\tilde{\eta})^{\mu \nu}\right\|_{H_{s, \delta}\left(\Omega_{\theta}\right)}$. From the expression (2.5) and (2.6) of $\gamma$ and the fact that $(\gamma-\tilde{\eta})^{\mu \nu},(\gamma-\tilde{\eta})_{\mu \nu} \in H_{s, \delta}\left(\Omega_{\theta}\right)$, we know that $\left(\alpha^{2}-1\right)$, $\left(\frac{1}{\alpha^{2}}-1\right), \beta^{i}, \frac{\beta^{i}}{\alpha^{2}},\left(g_{i j}-e_{i j}\right),\left(g^{i j}-\frac{\beta^{i} \beta^{j}}{\alpha^{2}}-e^{i j}\right) \in H_{s, \delta}\left(\Omega_{\theta}\right)$ with their norms bounded by $\|(\gamma-\tilde{\eta})\|_{H_{s, \delta}\left(\Omega_{\theta}\right)}$. So $\alpha^{2}$ is bounded both from below and above by certain constant. By Taylor's expansion $\left.|\alpha-1|=\mid \sqrt{1+\left(\alpha^{2}-1\right)}-1\right) \mid \leq$ $C\left|\alpha^{2}-1\right|$, hence is $L_{\delta}^{2}$ integrable. For higher order derivatives of $(\alpha-1)$, we can use the multiplication Lemma 2.6 and the bound of $\left(\alpha^{2}-1\right)$ to show that $D^{\mu}(\alpha-1)$ lies in $L_{s-|\mu|, \delta+|\mu|}\left(\Omega_{\theta}\right)$. So $(\alpha-1)$ lies in $H_{s, \delta}\left(\Omega_{\theta}\right)$ and has the norm bounded by a constant depending only on $\tilde{\lambda}$ and $\|(\gamma-$ $\tilde{\eta})^{\mu \nu} \|_{H_{s, \delta}\left(\Omega_{\theta}\right)} \cdot$

So the metric coefficients of out boost solution $\gamma$ satisfy that $\{(\alpha-1)$, $\left.\beta^{i}, \beta_{i}, g^{i j}-e^{i j}, g_{i j}-e_{i j}\right\} \in H_{s+1, \delta}\left(\Omega_{\theta}\right)$ with norms bounded by a constant

\footnotetext{
${ }^{20}$ See the constant $N, A$ and $B$ in Definition 11.8 on [6, page 397].
} 
depending only on the elliptic constant $\lambda$ of $g$ and $\|g-e\|_{H_{s+1, \delta+\frac{1}{2}}(\Sigma)}+$ $\|k\|_{H_{s, \delta+\frac{3}{2}}(\Sigma)}$. By the Sobolev embedding $H_{s+1, \delta}\left(\Omega_{\theta}\right) \subset C_{\kappa}^{2}\left(\Omega_{\theta}\right)$ for some $0<\kappa<\delta+2$, all the terms above are uniformly bounded.

Given $s_{1} \geq 3$ and $\delta_{1}>-2$. Let $\mathcal{B}_{\rho}$ be a ball of radius $\rho$ containing scalar functions in $H_{s_{1}+1, \delta_{1}-\frac{1}{2}}(\Sigma)$ with $\|u\|_{H_{s_{1}+1, \delta_{1}-\frac{1}{2}}(\Sigma)} \leq \rho$. We can choose $\rho$ small enough, such that after embedding $\|u\|_{C_{\kappa}^{2}(\Sigma)} \leq C \rho \leq \theta / 2$ for some $-1<\kappa<$ $\delta_{1}+1$, and,

$$
\text { Condition (A): } \quad|u(x)| \leq(\theta / 2)(\sigma(x))^{-\kappa}<(\theta / 2) \sigma(x) .
$$

So $\operatorname{Graph}_{u}=\{(x, u(x)): x \in \Sigma\}$ is a submanifold in $\Omega_{\theta}$. Furthermore, $|D u|_{e} \leq C \rho(\sigma(x))^{-(\kappa+1)}$. As $(\alpha-1), \beta,(g-e)$ are all uniformly bounded, we can then choose $\rho$ small enough so that:

Condition (B): $|D u|_{e} \leq \frac{1}{100},\left|\langle\beta, D u\rangle_{g}\right| \leq \frac{1}{2},|U|=\left|\frac{\alpha|D u|_{g}}{1+\langle\beta, D u\rangle_{g}}\right| \leq \frac{1}{2}$,

where $U$ is defined in (2.12). Then $G r a p h_{u}$ is spacelike and $\nu=\sqrt{1-|U|^{2}}$ is well-defined. So we can study the operator

$$
\mathcal{H}: u \rightarrow H_{u},
$$

where $H_{u}$ is the mean curvature of $\operatorname{Graph}_{u}$ given by (2.16).

Now we will show that composition is continuous as follows.

Lemma 4.2. Given $s_{1} \geq 3, \quad \delta_{1}>-2$ and $\theta \in(0,1)$. Consider $\mathcal{B}_{\rho} \subset$ $H_{s_{1}+1, \delta_{1}-\frac{1}{2}}(\Sigma)$ with $\rho$ small enough satisfying Condition $(A)$ as above for the $\theta$. Then the composition map:

$$
(f, u) \rightarrow \tilde{f}=f(x, u(x)+t),
$$

is a continuous map $H_{s^{\prime}, \delta^{\prime}}\left(\Omega_{\theta}\right) \times \mathcal{B}_{\rho} \rightarrow H_{s^{\prime}, \delta^{\prime}}\left(\Omega_{\theta / 2}\right)$, for $s^{\prime} \leq s_{1}+1$ and $\delta^{\prime} \in \mathbb{R}$. Furthermore, when restricted to Graph ,

$$
(f, u) \rightarrow f(x, u(x))
$$

is a continuous map $H_{s^{\prime}, \delta^{\prime}}\left(\Omega_{\theta}\right) \times \mathcal{B}_{\rho} \rightarrow H_{s^{\prime}-1, \delta^{\prime}+\frac{1}{2}}\left(\Sigma, \Omega_{\theta / 2}\right)$.

Proof. Condition (A) (4.1) implies that $|u(x)| \leq(\theta / 2) \sigma(x)^{-\kappa}$ for some $-1<$ $\kappa<\delta_{1}+1$, so we can consider a well-defined map $F: \Omega_{\theta} \rightarrow \Omega_{\frac{3}{2} \theta}$, where $F:(x, t) \rightarrow(x, u(x)+t)$. Then $\operatorname{det}(D F)=1$, so $F$ is a diffeomorphism $\Omega_{\theta} \rightarrow$ $F\left(\Omega_{\theta}\right)$. Furthermore, $(F-i d)(x, t)=(0, u(x)) \in H_{s_{1}+1, \delta_{1}}\left(\Omega_{\theta}\right)$. Now we can 
apply Lemma 2.7 to the mapping $F$, so $f \rightarrow \tilde{f}=f \circ F$ is an isomorphism $H_{s^{\prime}, \delta^{\prime}}\left(\Omega_{\theta}\right) \rightarrow H_{s^{\prime}, \delta^{\prime}}\left(F\left(\Omega_{\theta}\right)\right)$. In fact, by the bound of $u$, we know that $F\left(\Omega_{\theta}\right)$ contains $\Omega_{\theta / 2}$, so clearly $\tilde{f}$ lies in $H_{s^{\prime}, \delta^{\prime}}\left(\Omega_{\theta / 2}\right)$, and we have the continuity for the first factor $f$. For the second factor $u$, we only need to show that $u \rightarrow f(x, u(x)+t)$ is continuous $H_{s_{1}+1, \delta_{1}-\frac{1}{2}}(\Sigma) \rightarrow L_{\delta^{\prime}}^{2}\left(\Omega_{\theta / 2}\right)$ for fixed $f \in$ $L_{\delta^{\prime}}^{2}\left(\Omega_{\theta}\right)$. Using multiplication Lemma 2.6 recursively to higher derivatives as in the proof of [10, Theorem 2.3] gives the continuity in $H_{s^{\prime}, \delta^{\prime}}$. Suppose $u_{n} \rightarrow$ $u$ in $H_{s_{1}+1, \delta_{1}-\frac{1}{2}}$, hence $u_{n} \rightarrow u$ in $C_{\kappa}^{0}$ for some $-1<\kappa<\delta_{1}+1$. To show the $L_{\delta^{\prime}}^{2}$ continuity, we can approximate $f$ by compactly supported smooth function $g$ in $L_{\delta^{\prime}}^{2}$, then $\left|f\left(x, u_{n}(x)+t\right)-f(x, u(x)+t)\right| \leq \mid f\left(x, u_{n}(x)+t\right)-$ $g\left(x, u_{n}(x)+t\right)|+| g(x, u(x)+t)-f(x, u(x)+t)|+| g\left(x, u_{n}(x)+t\right)-$ $g(x, u(x)+t) \mid$. The first and second terms can be chosen very small in $L_{\delta^{\prime}}^{2}$, and the third one converge to 0 in $L_{\delta^{\prime}}^{2}$. So we get the continuity. For the restriction, we can directly apply the restriction Lemma 2.8 to $\tilde{f}$.

Moreover, we also have the differentiability w.r.t. $u$.

Lemma 4.3. Given $s_{1} \geq 3, \delta_{1}>-2, \theta \in(0,1), \delta^{\prime} \in \mathbb{R}$ and $f \in H_{s_{1}+1, \delta^{\prime}}\left(\Omega_{\theta}\right)$. Consider $\mathcal{B}_{\rho} \subset H_{s_{1}+1, \delta_{1}-\frac{1}{2}}(\Sigma)$ with $\rho$ chosen to satisfy Condition (A) in (4.1) for the $\theta$. Then

$$
\mathcal{F}: u \rightarrow f(x, u(x))
$$

is continuous Fréchet differentiable as a map $\mathcal{B}_{\rho} \rightarrow H_{s_{1}-1, \delta^{\prime}+\frac{1}{2}}(\Sigma)$. Furthermore, the Fréchet derivative is given by formal derivatives,

$$
D_{u} \mathcal{F}(v)=\partial_{t} f(x, u(x)) \cdot v
$$

where $v \in H_{s_{1}+1, \delta_{1}-\frac{1}{2}}(\Sigma)$.

Proof. Using Lemma 4.2, we know that $f(x, u(x)+t)$ lies in $H_{s_{1}+1, \delta^{\prime}}\left(\Omega_{\theta / 2}\right)$, and $f(x, u(x)) \in H_{s_{1}, \delta^{\prime}+\frac{1}{2}}\left(\Sigma, \Omega_{\theta / 2}\right)$. Hence $\partial_{t} f(x, t) \in H_{s_{1}, \delta^{\prime}+1}\left(\Omega_{\theta / 2}\right)$ and $\partial_{t} f(x, u(x)) \in H_{s_{1}-1, \delta^{\prime}+\frac{3}{2}}(\Sigma)$. To show that $\mathcal{F}$ is Fréchet differentiable (see [5, Definition 1.1.1]), we can first show that it is Gateaux differentiable (see [5, Definition 1.1.2]), i.e.,

$$
\lim _{\tau \rightarrow 0} \frac{\left\|f(x, u(x)+\tau v(x))-f(x, u(x))-\partial_{t} f(x, u(x))(\tau v(x))\right\|_{H_{s_{1}-1, \delta^{\prime}+\frac{1}{2}}(\Sigma)}}{\tau\|v(x)\|_{H_{s_{1}+1, \delta_{1}-\frac{1}{2}}(\Sigma)}}=0,
$$


for any $v \in H_{s_{1}+1, \delta_{1}-\frac{1}{2}}(\Sigma)$. Using Newton-Leibniz formula,

$$
f(x, u(x)+\tau v(x))-f(x, u(x))=\left(\int_{s=0}^{1} \partial_{t} f(x, u(x)+s \tau v(x)) d s\right)(\tau v(x)),
$$

Using the multiplication Lemma $(2.3)$ in the case $H_{s_{1}-1, \delta^{\prime}+\frac{3}{2}}(\Sigma) \times H_{s_{1}+1, \delta_{1}-\frac{1}{2}}$ $(\Sigma) \rightarrow H_{s_{1}-1, \delta^{\prime}+\frac{1}{2}}(\Sigma)$, we only need to show,

$$
\lim _{\tau \rightarrow 0}\left\|\partial_{t} f(x, u(x)+\tau v(x))-\partial_{t} f(x, u(x))\right\|_{H_{s_{1}-1, \delta^{\prime}+\frac{3}{2}}(\Sigma)}=0 .
$$

This convergence follows from the continuity of $\left(\partial_{t} f, u\right) \rightarrow \partial_{t} f(x, u(x))$ as a $\operatorname{map} H_{s_{1}, \delta^{\prime}+1}\left(\Omega_{\theta}\right) \times H_{s_{1}+1, \delta_{1}-\frac{1}{2}}(\Sigma) \rightarrow H_{s_{1}-1, \delta^{\prime}+\frac{3}{2}}(\Sigma)$ in Lemma 4.2. Now the multiplication operator $L_{u}: v \rightarrow \partial_{t} f(x, u(x)) \cdot v$ is a bounded linear operator in $L\left(H_{s_{1}+1, \delta_{1}-\frac{1}{2}}(\Sigma), H_{s_{1}-1, \delta^{\prime}+\frac{1}{2}}(\Sigma)\right)$ with

$$
\left\|L_{u}\right\|_{L\left(H_{s_{1}+1, \delta_{1}-\frac{1}{2}}(\Sigma), H_{s_{1}-1, \delta^{\prime}+\frac{1}{2}}(\Sigma)\right)} \leq C\left\|\partial_{t} f(x, u(x))\right\|_{H_{s_{1}-1, \delta^{\prime}+\frac{3}{2}}(\Sigma)}
$$

by inequality (2.3). The operator $L_{u}$ is also continuous w.r.t $u$ by Lemma 4.2, so we know that $\mathcal{F}$ is Fréchet differentiable by [5, Theorem 1.1.3], and $D_{u} \mathcal{F}(v)=\partial_{t} f(x, u(x)) \cdot v$.

Now we can prove the differentiability of $H_{u}$ w.r.t. $u$.

Proposition 4.4. For $s \geq 4, \delta>-2$. Given a vacuum data $(g, k) \in$ $\mathcal{V} \mathcal{C}_{s+1, \delta+\frac{1}{2}}(\Sigma)$ and the boost ratio $\theta$ as in the beginning of this section. If $\mathcal{B}_{\rho} \subset$ $H_{s, \delta-\frac{1}{2}}(\Sigma)$ with $\rho$ satisfying Conditions $(A)$ and $(B)$ as in (4.1) and (4.2) for the $\theta$, then the mean curvature operator (4.3) $\mathcal{H}: \mathcal{B}_{\rho} \rightarrow H_{s-2, \delta+\frac{3}{2}}(\Sigma)$ is continuously differentiable w.r.t. $u$, i.e., $\left(D_{u} \mathcal{H}\right) \in C\left(\mathcal{B}_{\rho}, L\left(H_{s, \delta-\frac{1}{2}}(\Sigma)\right.\right.$, $\left.\left.H_{s-2, \delta+\frac{3}{2}}(\Sigma)\right)\right)$. Furthermore, $D_{u} \mathcal{H}$ is given by the formal variational formula.

Proof. By the choice of $\rho, \mathcal{H}$ is well-defined. Write out the expression for $H_{u}$ in (2.16) in local coordinates $\left\{\left(t, x^{i}\right): i=1,2,3\right\}$ of $\Omega_{\theta}$ as follows:

$$
\begin{aligned}
H_{u}= & \left(g_{M}\right)^{i j}\left\langle\nabla_{\alpha_{i}} N, \alpha_{j}\right\rangle_{\gamma}=\nu \cdot\left(g_{M}\right)^{i j}\left\langle\nabla_{\partial_{i}+u_{i} \partial_{t}}(U+T), \partial_{j}+u_{j} \partial_{t}\right\rangle_{\gamma} \\
= & \nu \cdot\left(g_{M}\right)^{i j}\left\{\left(\partial_{i}+u_{i} \partial_{t}\right)(U+T)^{\mu}\left\langle\partial_{\mu}, \partial_{j}+u_{j} \partial_{t}\right\rangle_{\gamma}\right. \\
& \left.+(U+T)^{\mu}\left\langle\nabla_{\partial_{i}+u_{i} \partial_{t}} \partial_{\mu}, \partial_{j}+u_{j} \partial_{t}\right\rangle_{\gamma}\right\} \\
= & \nu \cdot\left(\gamma^{i j}+\frac{\nu^{2}}{\alpha^{2}}\left(\beta^{i}-\alpha U^{i}\right)\left(\beta^{j}-\alpha U^{j}\right)\right)\left\{\left(\partial_{i}+u_{i} \partial_{t}\right)\left(U^{\mu}+T^{\mu}\right)\right. \\
& \left.\cdot\left(\gamma_{\mu j}+u_{j} \gamma_{\mu t}\right)+\left(U^{\mu}+T^{\mu}\right)\left(\Gamma_{i \mu, j}+u_{i} \Gamma_{t \mu, j}+u_{j} \Gamma_{i \mu, t}+u_{i} u_{j} \Gamma_{t \mu, t}\right)\right\}
\end{aligned}
$$


where $\Gamma_{\mu \nu, \sigma}$ is the Christoffel symbol for $\gamma$, and all coefficients of $\gamma$ are evaluated at $(x, u(x))$. Except for the term $\nu, H_{u}$ is an algebraic expression containing two type of terms in (4.8). One type of terms are the composition of the coefficients of $(\gamma-\tilde{\eta})$ and $\partial \gamma$ with $(x, u(x))$, and the other terms contains $\partial u$ and $\partial^{2} u$. The only term appears in the denominator is $1+$ $\langle\beta, D u\rangle_{g}$, and $\left|\langle\beta, D u\rangle_{g}\right| \leq \frac{1}{2}$ by the choice of $\rho$ as in Condition (B).

Since $(\gamma-\tilde{\eta}) \in H_{s+1, \delta}\left(\Omega_{\theta}\right)$, the composition of the metric coefficients of $(\gamma-\tilde{\eta})$ with $(x, u(x))$, i.e., $\left\{\left(\gamma^{\mu \nu}-\tilde{\eta}^{\mu \nu}\right),\left(\gamma_{\mu \nu}-\tilde{\eta}_{\mu \nu}\right),(\alpha-1), \beta^{i}, \beta_{i},\left(g^{i j}-\right.\right.$ $\left.\left.e^{i j}\right),\left(g_{i j}-e_{i j}\right)\right\}(x, u(x))$ are continuously differentiable w.r.t. $u$ as maps $H_{s, \delta-\frac{1}{2}}(\Sigma) \rightarrow H_{s-2, \delta+\frac{1}{2}}(\Sigma)$ by Lemma 4.3. Similarly the composition of the coefficients of $\partial \gamma \operatorname{with}^{2}(x, u(x))$, i.e., $(\partial \gamma)(x, u(x))$ are also continuously differentiable w.r.t. $u$ as maps $H_{s, \delta-\frac{1}{2}}(\Sigma) \rightarrow H_{s-2, \delta+\frac{3}{2}}(\Sigma)$. The terms $\partial u$ and $\partial^{2} u$ are trivially continuous differentiable w.r.t. $u$ as maps $H_{s, \delta-\frac{1}{2}}(\Sigma) \rightarrow$ $H_{s-1, \delta+\frac{1}{2}}(\Sigma)$ and $H_{s, \delta-\frac{1}{2}}(\Sigma) \rightarrow H_{s-2, \delta+\frac{3}{2}}(\Sigma)$, respectively. Hence $U=$ $\frac{\alpha D u}{1+\langle\beta, D u\rangle} \in H_{s-1, \delta+\frac{1}{2}}(\Sigma)$ and is continuously differentiable w.r.t. $u$, hence is $\nu^{2}-1=\frac{|U|^{2}}{1-|U|^{2}} \in H_{s-1, \delta+\frac{3}{2}}(\Sigma)$, since $|U| \leq \frac{1}{2}$. So by similar argument as that for $\alpha$ in Lemma 4.1, $(\nu-1)$ is also continuous differentiable w.r.t. $u$ as $H_{s, \delta-\frac{1}{2}}(\Sigma) \rightarrow H_{s-2, \delta+\frac{3}{2}}(\Sigma)$. Combing all them together, $H_{u}$ is continuously differentiable w.r.t. $u$ by the multiplication Lemma 2.6.

\subsection{Linear theory}

Given a 3-dimensional manifold $(\Sigma, e)$ which is Euclidean at infinity. Let us give some results about linear elliptic operators which are asymptotic to the Laplacian $\triangle_{e}$ on $(\Sigma, e)$. Such type of elliptic operators have been widely studied in $[2,7,10,19]$.

Let $L$ be an operator on $(\Sigma, e)$ of the form:

$$
L u=\Sigma_{k=0}^{2} a_{k} \partial^{k} u
$$

with $u$ and $L u$ functions on $\Sigma$, satisfying:

$$
\begin{aligned}
& \lambda e \leq a_{2} \leq \lambda^{-1} e \text { as metrics, with } \lambda \text { the elliptic coefficient } \\
& \left(a_{2}-e\right) \in H_{s_{0}+1, \delta_{0}}(\Sigma), a_{1} \in H_{s_{0}, \delta_{0}+1}(\Sigma), a_{0} \in H_{s_{0}-1, \delta_{0}+2}(\Sigma)
\end{aligned}
$$

where $s_{0} \geq 4, \delta_{0}>-\frac{3}{2}$. We will show that in certain weighted spaces, such $L$ has uniformly bounded inverse on the orthogonal complement of $\operatorname{ker}(L)$ depending only on the norms of the coefficients. First we have, 
Lemma 4.5. Let $s_{0}, \delta_{0}$ be as in (4.9). Given $s \leq s_{0},-\frac{3}{2}<\delta<-\frac{1}{2}$. There exist a constant $C$ and a large $r>R$, depending only on $s_{0}, \delta_{0}$, the elliptic coefficient $\lambda$ and the norms $\left\|a_{2}-e\right\|_{H_{s_{0}, \delta_{0}}(\Sigma)},\left\|a_{1}\right\|_{H_{s_{0}-1, \delta_{0}+1}(\Sigma)}$ and $\left\|a_{0}\right\|_{H_{s_{0}-2, \delta_{0}+2}(\Sigma)}$, such that for any $u \in H_{s, \delta-1}(\Sigma)$,

$$
\|u\|_{H_{s, \delta-1}(\Sigma)} \leq C\left(\|L u\|_{H_{s-2, \delta+1}(\Sigma)}+\|u\|_{H_{s-2}\left(\Sigma_{\text {int }, 2 r}\right)}\right),
$$

where $\Sigma_{\text {int }, 2 r}$ is the union of $\Sigma_{\text {int }}$ with all the annuli $B_{2 r} \backslash B_{R}$ inside each end $\Sigma_{i}$, and $H_{s-2}$ is the standard $L^{2}$ Sobolev space on $\Sigma_{\mathrm{int}, 2 r}$.

Proof. Let $\Sigma=\Sigma_{\text {int }} \cup_{i=1}^{l} E_{i}$. Given a function $\chi \in C^{\infty}\left(\mathbb{R}^{3} \backslash B_{1}\right)$, such that $0 \leq \chi \leq 1, \chi \equiv 1$ on $\mathbb{R}^{3} \backslash B_{2}$ and $\chi \equiv 0$ near $\partial B_{1}$. We can find a partition of unity $\left\{\chi_{i, r}\right\}_{i=0}^{l}$ of $\Sigma$ for $r>R$, with $\chi_{i, r}(x)=\chi(x / r)$ for $x \in E_{i} \cong \mathbb{R}^{3} \backslash$ $B_{R}$, and $\chi_{i, r}(x)=0$ for $x \in \Sigma \backslash E_{i}$, and $\chi_{0, r}(x)=1-\Sigma_{i=1}^{l} \chi_{i, r}(x)$. Then $u=$ $\sum_{i=1}^{l} u_{i, r}$, with $u_{i, r}=\chi_{i, r} u$. Let us fix an end $E_{i}$ and $u_{i, r}$ and forget the subindex $i$ now. Since $-\frac{3}{2}<\delta<-\frac{1}{2}$ corresponds to non-exceptional value in [2], we can apply [2, Theorem 1.7] with $p=2$ here,

$$
\begin{aligned}
\left\|u_{r}\right\|_{H_{s, \delta-1}\left(\mathbb{R}^{3}\right)} & \leq C_{1}\left\|\Delta u_{r}\right\|_{H_{s-2, \delta+1}\left(\mathbb{R}^{3}\right)} \\
& \leq C_{1}\left\{\left\|L u_{r}\right\|_{H_{s-2, \delta+1}(E)}+\left\|(L-\triangle) u_{r}\right\|_{H_{s-2, \delta+1}(E)}\right\},
\end{aligned}
$$

where $\triangle$ is the Laplacian operator w.r.t. $\delta_{i j}$ and $C_{1}$ a uniform constant.

$$
\begin{aligned}
\left\|L u_{r}\right\|_{H_{s-2, \delta+1}(E) \leq} \leq & \left\|\chi_{r} L u\right\|_{H_{s-2, \delta+1}(E)}+\| 2 a_{2}^{i j} \partial_{i} u \partial_{j} \chi_{r} \\
& +\left(a_{2}^{i j} \partial^{2} \chi_{r}+a_{1}^{i} \partial \chi_{r}\right) u \|_{H_{s-2, \delta+1}(E)} \\
\leq & C_{2}(r)\left(\|L u\|_{H_{s-2, \delta+1}(E)}+\|u\|_{H_{s-1}\left(A_{r}\right)}\right)
\end{aligned}
$$

with $A_{r}=B_{2 r} \backslash B_{r}$, and $C_{2}(r)$ is a constant depending only on $r$ and $\| a_{2}-$ $e\left\|_{H_{s_{0}, \delta_{0}}\left(A_{r}\right)},\right\| a_{1} \|_{H_{s_{0}-1, \delta_{0}+1}\left(A_{r}\right)}$. Since $\delta_{0}>-\frac{3}{2}$, there exists some $\epsilon>0$, such that $\delta_{1}=\delta_{0}-\epsilon>-\frac{3}{2}$. Using the multiplication Lemma 2.5,

$$
\begin{aligned}
\left\|(L-\triangle) u_{r}\right\|_{H_{s-2, \delta+1}\left(E_{r}\right)}= & \left\|\left(a_{2}^{i j}-\delta^{i j}\right) \partial^{2} u_{r}+a_{1}^{i} \partial u_{r}+a_{0} u_{r}\right\|_{H_{s-2, \delta+1}\left(E_{r}\right)} \\
& \leq C_{3}\left(\left\|a_{2}-e\right\|_{H_{s, \delta_{1}}\left(E_{r}\right)}+\left\|a_{1}\right\|_{H_{s-1, \delta_{1}+1}\left(E_{r}\right)}\right. \\
& \left.+\left\|a_{0}\right\|_{H_{s-2, \delta_{1}+2}\left(E_{r}\right)}\right)\left\|u_{r}\right\|_{H_{s, \delta-1}\left(E_{r}\right)}
\end{aligned}
$$

where $E_{r}=\mathbb{R}^{3} \backslash B_{r}$ and $C_{3}$ a uniform constant. Now $\left\|a_{2}-e\right\|_{H_{s, \delta_{1}}\left(E_{r}\right)}+$ $\left\|a_{1}\right\|_{H_{s-1, \delta_{1}+1}\left(E_{r}\right)}+\left\|a_{0}\right\|_{H_{s-2, \delta_{1}+2}\left(E_{r}\right)} \leq\left(\left\|a_{2}-e\right\|_{H_{s, \delta_{0}}\left(E_{r}\right)}+\left\|a_{1}\right\|_{H_{s-1, \delta_{0}+1}\left(E_{r}\right)}+\right.$ $\left.\left\|a_{0}\right\|_{H_{s-2, \delta_{0}+2}\left(E_{r}\right)}\right) r^{-\epsilon}$ for $r$ large enough. So we can always choose a $r>R$, depending only on $\delta_{0}$ and $\left(\left\|a_{2}-e\right\|_{H_{s, \delta_{0}}\left(E_{r}\right)}+\left\|a_{1}\right\|_{H_{s-1, \delta_{0}+1}\left(E_{r}\right)}+\right.$ 
$\left.\left\|a_{0}\right\|_{H_{s-2, \delta_{0}+2}\left(E_{r}\right)}\right)$, such that $\left\|(L-\triangle) u_{r}\right\|_{H_{s-2, \delta+1}\left(E_{r}\right)} \leq \frac{1}{2 C_{1}}\left\|u_{r}\right\|_{H_{s, \delta-1}\left(E_{r}\right)}$. Putting them back to inequality (4.11), we get

$$
\left\|u_{r}\right\|_{H_{s, \delta-1}(\Sigma)} \leq C_{4}\left\{\|L u\|_{H_{s-2, \delta+1}(E)}+\|u\|_{H_{s-1}\left(A_{r}\right)}\right\},
$$

where $C_{4}$ depends only on $C_{2}(r)$. Using an interpolation inequality (see [7, Lemma 2.2]) to $\|u\|_{H_{s-1}\left(A_{r}\right)}$, we can get the estimate of (4.10) on each end. Applying the standard $L^{2}$ estimates to $u_{0, r}$ on $\Sigma_{\text {int }, 2 r}$ (see [6, page 547, Corollary 2.2]),

$$
\left\|u_{0, r}\right\|_{H_{s}\left(\Sigma_{\mathrm{int}, 2 r}\right)} \leq C_{5}\left\{\left\|L u_{0, r}\right\|_{H_{s-2}\left(\Sigma_{\mathrm{int}, 2 r}\right)}+\left\|u_{0, r}\right\|_{H_{s-2}\left(\Sigma_{\mathrm{int}, 2 r}\right)}\right\},
$$

where $C_{5}$ depends only on $s_{0}$, the elliptic coefficient $\lambda$ and the norms $\| a_{2}-$ $e\left\|_{H_{s}\left(\Sigma_{\mathrm{int}, 2 r}\right)},\right\| a_{1} \|_{H_{s-1}\left(\Sigma_{\mathrm{int}, 2 r}\right)}$ and $\left\|a_{0}\right\|_{H_{s-2}\left(\Sigma_{\mathrm{int}, 2 r}\right)}$. Combing results on all ends $E_{i, r}$ and $\Sigma_{\text {int,2r }}$ together, we can get (4.10) with $r$ and constant $C$ satisfying the requirement.

Now we can prove a lemma similar to $[2$, Theorem 1.10] and [19, Theorem 5.6].

Lemma 4.6. Let $s_{0}, \delta_{0}$ be as in (4.9). Given $s \leq s_{0},-\frac{3}{2}<\delta<-\frac{1}{2}$, the operator $L$ is a Fredholm operator:

$$
H_{s, \delta-1}(\Sigma) \rightarrow H_{s-2, \delta+1}(\Sigma),
$$

i.e., $L$ has finite-dimensional kernel $\operatorname{ker}(L, \delta-1)=\left\{v \in H_{s, \delta-1}(\Sigma)\right.$ : $L v=0\}$, and finite-dimensional co-kernel coker $(L, \delta-1)$.

Proof. From the multiplication Lemma 2.5, we know that $L$ is a bounded linear map $H_{s, \delta-1}(\Sigma) \rightarrow H_{s-2, \delta+1}(\Sigma)$. Standard argument using inequality (4.10) as in [2, Theorem 1.10] shows that the null space $N(L)$ is finitedimensional and $L$ has closed range. So $L$ is semi-Fredholm.

To show that $L$ has finite-dimensional co-kernel, we will borrow the techniques in [19, Theorem 5.6]. First, inequality (4.13) shows that the operator norm of $(L-\triangle): H_{s, \delta-1}\left(E_{r}\right) \rightarrow H_{s-2, \delta+1}\left(E_{r}\right)$ is $o(1)$ as $r \rightarrow \infty$. So for large enough $r$, the fact that $\triangle$ is Fredholm by [2, Theorem 1.7] and that the Fredholm property is open w.r.t operator norms [16, Proposition 16.35] show that $L_{i}=\triangle+\chi_{i, r}(L-\triangle)$ is Fredholm on $\mathbb{R}^{3}$, where clearly $L_{i}=L$ on $E_{2 r}$. By [16, Theorem 16.32] there exists a bounded linear operator $S_{i}: H_{s-2, \delta+1}\left(\mathbb{R}^{3}\right) \rightarrow H_{s, \delta-1}\left(\mathbb{R}^{3}\right)$, such that $L_{i} S_{i}=i d+K_{i}$ with $K_{i}$ a compact operator. Now $L: H_{s, \delta-1}\left(\Sigma_{\text {int }, 8 r}\right) \rightarrow H_{s-2, \delta+1}\left(\Sigma_{\text {int }, 8 r}\right)$ is Fredholm since 
$\Sigma_{\mathrm{int}, 8 r}$ is compact, so there exists a Fredholm inverse $S_{0}: H_{s-2, \delta+1}\left(\Sigma_{\mathrm{int}, 8 r}\right) \rightarrow$ $H_{s, \delta-1}\left(\Sigma_{\text {int }, 8 r}\right)$, such that $L S_{0}=i d+K_{0}$, for $K_{0}$ compact operator. Define

$$
S u=\chi_{0,4 r} S_{0} u_{0,8 r}+\Sigma_{i=1}^{l} \chi_{i, 2 r} S_{i} u_{i, r},
$$

which is a bounded linear operator $H_{s-2, \delta+1}(\Sigma) \rightarrow H_{s, \delta-1}(\Sigma)$. Then a calculation as in $[19,(5.6 .5)]$ shows that $L S=i d+K$, where $K$ is a compact operator. Hence $L$ has finite-dimensional co-kernel.

The Fredholm index of $L$ is defined to be:

$$
i(L, \delta-1)=\operatorname{dim} \operatorname{ker}(L, \delta-1)-\operatorname{dim} \operatorname{coker}(L, \delta-1) .
$$

By comparing the index of $L$ to that of the Laplacian $\triangle_{e}$ of $e$, we can show that $L$ is surjective when $a_{0} \leq 0$.

Lemma 4.7. Let $s_{0}, \delta_{0}$ be as in (4.9). Given $s \leq s_{0},-\frac{3}{2}<\delta<-\frac{1}{2}$. Suppose $a_{0} \leq 0$, then $L$ is surjective. Furthermore, $\operatorname{dim} \operatorname{ker}(L, \delta-1)=d_{\delta-1}=$ dim $\operatorname{ker}\left(\triangle_{e}, \delta-1\right)$. If we denote $\operatorname{ker}(L, \delta-1)^{\perp}$ to be the orthogonal complement of $\operatorname{ker}(L, \delta-1)$ w.r.t the $L_{\delta-1}^{2}$ inner product $\langle\cdot, \cdot\rangle_{L_{\delta-1}^{2}(\Sigma)}$ as in Definition 2.3, then:

$$
L: \operatorname{ker}(L, \delta-1)^{\perp} \rightarrow H_{s-2, \delta+1}(\Sigma)
$$

is an isomorphism.

Proof. Since $L$ can be joined continuously to $\triangle_{e}$ by $L_{t}=t L+(1-t) \triangle_{e}$, we know $i(L, \delta-1)=i\left(\triangle_{e}, \delta-1\right)$. [19, Theorem 6.2] says that $\triangle_{e}$ is surjective when $\delta-1<-\frac{1}{2}$. In order to show that $L$ is surjective, or equivalently $\operatorname{dim} \operatorname{coker}(L, \delta-1)=0$, we only need to show that $\operatorname{dim} \operatorname{ker}(L, \delta-1) \leq$ $\operatorname{dim} \operatorname{ker}\left(\triangle_{e}, \delta-1\right)$. This comes from the asymptotical expansion given in [2] as follows. For $u \in \operatorname{ker}(L, \delta-1)$, by [2, Theorem 1.17], $L u=0$ implies that on each end $E_{i}$, there exists a harmonic homogenous function $h_{k}$ of order $k \leq k(\delta-1)$, where $k(\tilde{\delta})=\max \left\{k \in \mathbb{Z}: k \leq-\left(\tilde{\delta}+\frac{3}{2}\right)\right\}^{21}$, such that $u=h_{k}+o\left(r^{k-\beta}\right)$ for $0<\beta<\delta+\frac{3}{2}$. In our case, $k(\delta-1)=0$. In fact, if $u \neq 0$, there must exist at least one end, on which $k \geq 0$. Or the decay implies that $u=o(1)$ at infinity on $\Sigma$, so $u=0$ by maximum principle since $a_{0} \leq 0$. So $\operatorname{dim} \operatorname{ker}(L, \delta-1)$ is less or equal than the the number of linearly independent harmonic polynomials of order $\leq k(\delta-1)$ multiplied with the

\footnotetext{
${ }^{21}$ See the definition for $k(\delta)$ in [2, Equation (1.21)]. Their $\delta$ is the same as $-\left(\delta+\frac{3}{2}\right)$ here.
} 
number of ends. It is easy to see that the basis of $k e r\left(\triangle_{e}, \delta-1\right)$ just consists of functions which have main part as the harmonic polynomial of oder $\leq$ $k(\delta-1)$ on one end, and $O(1 / r)$ parts in other ends. So the leading terms shows that $\operatorname{dim} \operatorname{ker}(L, \delta-1) \leq \operatorname{dim} \operatorname{ker}\left(\triangle_{e}, \delta-1\right)$. The isomorphism on the orthogonal complement follows from the fact that $L$ is bijective.

In fact, we can show a uniform norm bound for the inverse of $L$ on $\operatorname{ker}(L, \delta-1)^{\perp}$.

Lemma 4.8. Let $s_{0}, \delta_{0}$ be as in (4.9). Given $s \leq s_{0},-\frac{3}{2}<\delta<-\frac{1}{2}$. Suppose $a_{0} \leq 0$. Denote the inverse of $L: \operatorname{ker}(L, \delta-1)^{\perp} \rightarrow H_{s-2, \delta+1}(\Sigma)$ by $L^{-1}$, then there exists a constant $C$ depending only on $s_{0}, \delta_{0}$, the elliptic coefficient $\lambda$ and the norms $\left\|a_{2}-e\right\|_{H_{s_{0}+1, \delta_{0}}(\Sigma)},\left\|a_{1}\right\|_{H_{s_{0}, \delta_{0}+1}(\Sigma)}$ and $\left\|a_{0}\right\|_{H_{s_{0}-1, \delta_{0}+2}(\Sigma)}$, such that for any $v \in H_{s-2, \delta+1}(\Sigma)$,

$$
\left\|L^{-1} v\right\|_{H_{s, \delta-1}(\Sigma)} \leq C\|v\|_{H_{s-2, \delta+1}(\Sigma)}
$$

Proof. We only need to show that for any $u \in \operatorname{ker}(L, \delta-1)^{\perp}$,

$$
\|u\|_{H_{s, \delta-1}(\Sigma)} \leq C_{1}\|L u\|_{H_{s-2, \delta+1}(\Sigma)}
$$

for a uniform constant $C_{1}$ depending only on $s_{0}, \delta_{0}$, the elliptic coefficient $\lambda$, and the norms $\left\|a_{2}-e\right\|_{H_{s_{0}+1, \delta_{0}}(\Sigma)},\left\|a_{1}\right\|_{H_{s_{0}, \delta_{0}+1}(\Sigma)}$, and $\left\|a_{0}\right\|_{H_{s_{0}-1, \delta_{0}+2}(\Sigma)}$. By contradiction argument, suppose that the statement is wrong, which means that there exists a sequence of operators $L_{i}$ with $a_{i, 0} \leq 0$, uniformly bounded elliptic coefficient $\lambda_{i} \geq \lambda_{0}>0$ and uniformly bounded coefficients $\left\|a_{i, 2}-e\right\|_{H_{s_{0}+1, \delta_{0}}(\Sigma)},\left\|a_{i, 1}\right\|_{H_{s_{0}, \delta_{0}+1}(\Sigma)},\left\|a_{i, 0}\right\|_{H_{s_{0}-1, \delta_{0}+2}(\Sigma)} \leq C_{0}$, and a sequence of functions $u_{i} \in \operatorname{ker}\left(L_{i}, \delta-1\right)^{\perp}$, such that $\left\|u_{i}\right\|_{H_{s, \delta-1}(\Sigma)} \geq$ $i\left\|L_{i} u_{i}\right\|_{H_{s-2, \delta+1}(\Sigma)}$. By re-normalizing, we get a sequence of functions $u_{i}$, with $\left\|u_{i}\right\|_{H_{s, \delta-1}(\Sigma)}=1$, while $\left\|L_{i} u_{i}\right\|_{H_{s-2, \delta+1}(\Sigma)} \rightarrow 0$. By weak compactness, there exists a subsequence, which we still denote by $L_{i}$, such that the coefficients of $L_{i}$ converges weakly to that of a linear operator $L_{\infty}$ with $\lambda_{0} e \leq a_{\infty, 2} \leq \lambda_{0}^{-1} e$, $a_{\infty, 0} \leq 0$ and $\left\|a_{\infty, 2}-e\right\|_{H_{s_{0}+1, \delta_{0}}(\Sigma)},\left\|a_{\infty, 1}\right\|_{H_{s_{0}, \delta_{0}+1}(\Sigma)},\left\|a_{\infty, 0}\right\|_{H_{s_{0}-1, \delta_{0}+2}(\Sigma)} \leq$ $C_{0}$. Using inequality (4.10), there is a uniform constant $C_{2}$,

$$
\begin{aligned}
\left\|u_{i}-u_{j}\right\|_{H_{s, \delta-1}(\Sigma)} \leq & C_{2}\left(\left\|L_{i}\left(u_{i}-u_{j}\right)\right\|_{H_{s-2, \delta+1}(\Sigma)}+\left\|u_{i}-u_{j}\right\|_{H_{s-2}\left(\Sigma_{\mathrm{int}, 2 r}\right)}\right) \\
\leq & C_{2}\left(\left\|L_{i} u_{i}\right\|_{H_{s-2, \delta+1}(\Sigma)}+\left\|\left(L_{i}-L_{j}\right) u_{j}\right\|_{H_{s-2, \delta+1}(\Sigma)}\right. \\
& \left.+\left\|L_{j} u_{j}\right\|_{H_{s-2, \delta+1}(\Sigma)}+\left\|u_{i}-u_{j}\right\|_{H_{s-2}\left(\Sigma_{\mathrm{int}, 2 r}\right)}\right)
\end{aligned}
$$

Now $\quad\left\|\left(L_{i}-L_{j}\right) u_{j}\right\|_{H_{s-2, \delta+1}(\Sigma)} \leq C\left(\left\|a_{2, i}-a_{2, j}\right\|_{H_{s_{0}, \delta^{\prime}-1}(\Sigma)}+\| a_{1, i}-\right.$ $\left.a_{1, j}\left\|_{H_{s_{0}-1, \delta^{\prime}}(\Sigma)}+\right\| a_{0, i}-a_{0, j} \|_{H_{s_{0}-2, \delta^{\prime}+1}(\Sigma)}\right)$ for some $\delta_{0}>\delta^{\prime}>-\frac{3}{2}$ by the 
multiplication Lemma 2.6. The compact embedding ([7, Lemma 2.1]) of $H_{s_{0}+1-k, \delta_{0}-1+k}(\Sigma) \subset H_{s_{0}-k, \delta^{\prime}-1+k}(\Sigma) \quad$ for $\quad k=0,1,2$ imply that $\left\|\left(L_{i}-L_{j}\right) u_{j}\right\|_{H_{s-2, \delta+1}(\Sigma)} \rightarrow 0$ for a subsequence of $\left\{L_{i}\right\}$. Together with the compactness of $H_{s, \delta-1}(\Sigma) \subset H_{s-2}\left(\Sigma_{\text {int }, 2 r}\right)$, there exists a subsequence, which we still denote by $u_{i}$, such that $u_{i}$ converge strongly in $H_{s, \delta-1}(\Sigma)$ to a function $u_{\infty}$, with $\left\|u_{\infty}\right\|_{H_{s, \delta-1}(\Sigma)}=1$. Furthermore we have that $L_{\infty} u_{\infty}=0$ weakly by the weak convergence, and hence strongly in $H_{s-2, \delta+1}(\Sigma)$ by elliptic regularity.

By Lemma 4.7, we know that $\operatorname{dimker}\left(L_{i}, \delta-1\right) \equiv d_{\delta-1}$. We claim that $\operatorname{ker}\left(L_{i}, \delta-1\right)$ converge to a $d_{\delta-1}$-dimensional linear subspace of $\operatorname{ker}\left(L_{\infty}\right.$, $\delta-1)$. Let $\left\{v_{i, a}\right\}_{a=1}^{d_{\delta-1}}$ be an $L_{\delta-1}^{2}$ orthonormal basis for $\operatorname{ker}\left(L_{i}, \delta-1\right)$, with $\left\|v_{i, a}\right\|_{H_{s, \delta-1}(\Sigma)}=1$. By equation (4.18),

$$
\begin{aligned}
\left\|v_{i, a}-v_{j, a}\right\|_{H_{s, \delta-1}(\Sigma)} \leq & C\left(\left\|\left(L_{i}-L_{j}\right) v_{j, a}\right\|_{H_{s-2, \delta+1}(\Sigma)}\right. \\
& \left.+\left\|v_{i, a}-v_{j, a}\right\|_{H_{s-2}\left(\Sigma_{\text {int }, 2 r}\right)}\right) .
\end{aligned}
$$

Similar argument as above implies that a subsequence of $v_{i, a}$ converge strongly in $H_{s, \delta-1}(\Sigma)$ to some $v_{\infty, a}$. Hence $v_{\infty, a} \in \operatorname{ker}\left(L_{\infty}, \delta-1\right)$, and $\left\{v_{\infty, a}\right\}_{a=1}^{d_{\delta-1}}$ are also orthogonal in $L_{\delta-1}^{2}$ with $\left\|v_{\infty, a}\right\|_{H_{s, \delta-1}(\Sigma)}=1$. Since $L_{\infty}$ satisfies all the requirement of Lemma 4.7, $\operatorname{dimker}\left(L_{\infty}, \delta-1\right)=d_{\delta-1}$. Hence the limit of $\operatorname{ker}\left(L_{i}, \delta-1\right)$ is exactly the entire $\operatorname{ker}\left(L_{\infty}, \delta-1\right)$. As $u_{i}$ is perpendicular to $\operatorname{ker}\left(L_{i}, \delta-1\right)$ in $L_{\delta-1}^{2}$, passing to the limit, we know that $u_{\infty}$ is perpendicular to $\operatorname{ker}\left(L_{\infty}, \delta-1\right)$ in $L_{\delta-1}^{2}$ too, which is a contradiction to that $\left\|u_{\infty}\right\|_{H_{s, \delta-1}(\Sigma)}=1$ and $L_{\infty} u_{\infty}=0$. So we finish the proof.

\subsection{Existence of maximal data}

Now let us calculate the linearization of $\mathcal{H}$ with respect to $u$ at $(g, k, 0)$. Fix a vacuum data $(g, k) \in \mathcal{V} \mathcal{C}_{s+2, \delta+\frac{1}{2}}(\Sigma)$ with the unique boost solution $(\mathcal{V}, \gamma)$ given by Theorem 3.7. Recall the form (2.5) of $\gamma$ in local coordinates $\left(x^{i}, t\right)$ of $\Omega_{\theta}$. According to the initial data equations (3.5) and (3.6) for $\gamma$, the coefficients restricted to $t=0$ slice are given by:

$$
\left.\alpha\right|_{\Sigma} \equiv 1 ;\left.\quad \beta\right|_{\Sigma} \equiv 0
$$

In fact, our choice of $\left.\alpha\right|_{\Sigma}$ and $\left.\beta\right|_{\Sigma}$ implies that $\left.\partial_{t}\right|_{\Sigma}$ is the unit normal of $\Sigma$. Now recall the second variational formula for the mean curvature in $[1$, Section 2]. Let $X$ be a vector field in a neighborhood of $\Sigma$ with associated 
flow $\phi_{s}: \mathcal{V} \rightarrow \mathcal{V}$. Denote $H(s)$ by the mean curvature of $\phi_{s}(\Sigma)$, then

$$
\left.\partial_{s}(H(s))\right|_{s=0}=-\triangle_{g}\langle X, N\rangle+\langle X, N\rangle\left(|k|_{g}^{2}+\operatorname{Ric}_{\gamma}(N, N)\right)+\left\langle X, \nabla_{g} H\right\rangle,
$$

where $N$ is the unit normal of $\Sigma$ insider $\mathcal{V}$, and $R i c_{\gamma}$ the Ricci curvature of $\gamma$. In our case, $\operatorname{Ric}_{\gamma} \equiv 0$ by (1.3) since our $(\mathcal{V}, \gamma)$ is vacuum, and the unit normal $N=\partial_{t}$ on $\Sigma$. We can choose the vector field to be $X=v \partial_{t}$, where $v$ is a compactly supported smooth scalar function, so $\left\langle X, \nabla_{g} H\right\rangle=0$. Then $\left.\partial_{s} H(s)\right|_{s=0}$ is the linearization of $H$ w.r.t $u$, and $\langle X, N\rangle=-v$. Now combining all and using Proposition 4.4, we have,

Lemma 4.9. Using notations in Proposition 4.4, the Fréchet derivative of $\mathcal{H}(g, k, u)$ with respect to the factor $u$ at a vacuum data $(g, k, 0)$ is a linear operator $L_{0}: H_{s, \delta-\frac{1}{2}}(\Sigma) \rightarrow H_{s-2, \delta+\frac{3}{2}}(\Sigma)$ given by:

$$
\left(D_{v} \mathcal{H}\right)_{(g, k, 0)}=L_{0} v=\left(\triangle_{g}-|k|_{g}^{2}\right) v .
$$

Now let us focus on the operator $L_{0} . L_{0}$ is in fact Fredholm and surjective by Lemmas 4.6 and 4.7. By making use the fact that $L_{0}$ has finitedimensional kernel and is surjective, we can get the existence of solutions of $\mathcal{H}(g, k, u)=0$ for $(g, k)$ with small trace $t r_{g} k$ by a perturbation method, but no uniqueness due to the existence of non-trivial kernel $\operatorname{ker}\left(L_{0}, \delta-\frac{1}{2}\right)$. We will give an existence and uniqueness theorem in the orthogonal complement of the kernel in order to find symmetry preserving solutions in the following section. Let us first give a Quantitative Inverse Function Theorem motivated by [20].

Theorem 4.10. Let $X, Y$ be Banach spaces, and $U \subset X$ an open set. Suppose $F: U \rightarrow Y$ is a continuous map, and has Fréchet derivative w.r.t. $x$, such that $\frac{\partial F}{\partial x}$ is continuous. For a point $x_{0} \in U$, with $F\left(x_{0}\right)=y_{0}$. Suppose $\frac{\partial F}{\partial x}\left(x_{0}\right): X \rightarrow Y$ is invertible, and $\left\|\left[\frac{\partial F}{\partial x}\left(x_{0}\right)\right]^{-1}\right\| \leq C$. Assume that we can find $r_{0}>0$, such that for any $x \in B_{r_{0}}\left(x_{0}\right) \subset U$,

$$
\left\|\frac{\partial F}{\partial x}(x)-\frac{\partial F}{\partial x}\left(x_{0}\right)\right\| \leq \frac{1}{2 C} .
$$

Then for any $y \in Y$ with

$$
\left|y-y_{0}\right|_{Y}<\frac{r_{0}}{2 C}
$$

there exists a unique $x \in B_{r_{0}}\left(x_{0}\right)$, such that $F(x)=y$. 
Proof. Fix a $y \in B_{r_{0} / 2 C}\left(y_{0}\right) \subset Y$. Let us consider the map $T: B_{r_{0}}(0) \subset X \rightarrow$ $X$, defined by

$$
T(x)=x-\left[\frac{\partial F}{\partial x}\left(x_{0}\right)\right]^{-1}\left(F\left(x_{0}+x\right)-y\right)
$$

$x$ is a fixed point if and only if $F\left(x_{0}+x\right)=y$. So let us use the Banach Fixed Point Theorem to find a fixed point for $T$ in $B_{r_{0}}(0)$. First, for any $x_{1}, x_{2} \in B_{r_{0}}(0)$,

$$
\begin{aligned}
\mid T\left(x_{1}\right) & -\left.T\left(x_{2}\right)\right|_{X} \\
& =\left|\left(x_{1}-x_{2}\right)-\left[\frac{\partial F}{\partial x}\left(x_{0}\right)\right]^{-1}\left(F\left(x_{0}+x_{1}\right)-F\left(x_{0}+x_{2}\right)\right)\right|_{X} \\
& \leq\left\|\left[\frac{\partial F}{\partial x}\left(x_{0}\right)\right]^{-1}\right\| \cdot\left|\frac{\partial F}{\partial x}\left(x_{0}\right)\left(x_{1}-x_{2}\right)-\frac{\partial F}{\partial x}\left(x_{0}+\bar{x}\right)\left(x_{1}-x_{2}\right)\right|_{Y} \\
& \leq C\left\|\frac{\partial F}{\partial x}\left(x_{0}\right)-\frac{\partial F}{\partial x}\left(x_{0}+\bar{x}\right)\right\| \cdot\left|x_{1}-x_{2}\right|_{X} \\
& \leq C \frac{1}{2 C}\left|x_{1}-x_{2}\right|_{X} \leq \frac{1}{2}\left|x_{1}-x_{2}\right|_{X},
\end{aligned}
$$

where we used the mean value theorem to estimate $\left(F\left(x_{0}+x_{1}\right)-F\left(x_{0}+\right.\right.$ $\left.x_{2}\right)$ ) in the first " $\leq$ ", and condition $(4.22)$ in the third " $\leq$ ". So $T$ is a contraction map on $B_{r_{0}}(0)$. Next, for any $x \in B_{r_{0}}(0)$, and $\left|y-y_{0}\right|_{Y}<\frac{r_{0}}{2 C}$,

$$
\begin{aligned}
|T(x)|_{X} & \\
& \leq\left\|\left[\frac{\partial F}{\partial x}\left(x_{0}\right)\right]^{-1}\right\| \cdot\left|\frac{\partial F}{\partial x}\left(x_{0}\right) x-\left(F\left(x_{0}+x\right)-F\left(x_{0}\right)\right)-\left(y-F\left(x_{0}\right)\right)\right|_{Y} \\
& \leq C\left(\left|\left(\frac{\partial F}{\partial x}\left(x_{0}\right)-\frac{\partial F}{\partial x}\left(x_{0}+\bar{x}\right)\right) x\right|_{X}+\left|y-F\left(x_{0}\right)\right|_{Y}\right) \\
& \leq C\left(\left\|\frac{\partial F}{\partial x}\left(x_{0}\right)-\frac{\partial F}{\partial x}\left(x_{0}+\bar{x}\right)\right\| \cdot|x|_{X}+\left|y-F\left(x_{0}\right)\right|_{Y}\right) \\
& <C\left(\frac{1}{2 C} r_{0}+\frac{r_{0}}{2 C}\right)=r_{0}
\end{aligned}
$$

where we use condition (4.22) in the last " $<$ ". So $T$ maps $B_{r_{0}}(0)$ to $B_{r_{0}}(0)$. By applying the Banach Fixed Point Theorem (or Contraction Mapping Theorem) to $T: B_{r_{0}}(0) \rightarrow B_{r_{0}}(0)$, we finish the proof. 
Remark 4.11. This can be viewed as a careful reworking of the proof of [5, Theorem 1.2.1]. Theorem 3.1 and Theorem 3.2 in [20] also gave a proof about the quantitative inverse function theorem.

Theorem 4.12. For $s \geq 4,-2<\delta<-1$. Fix a 3-manifold $(\Sigma, e)$ which is Euclidean at infinity and a $\lambda>0$. Given a vacuum data $(g, k) \in \mathcal{V C}_{s+2, \delta+\frac{1}{2}}(\Sigma)$, with $g \geq \lambda e$, there exist $\epsilon>0$ and $\rho^{\prime}>0$ small enough, depending only on the norms $\|g-e\|_{H_{s+2, \delta+\frac{1}{2}}(\Sigma)}+\|k\|_{H_{s+1, \delta+\frac{3}{2}}(\Sigma)}$ and the elliptic constant $\lambda$, such that if $\left\|t r_{g} k\right\|_{H_{s-2, \delta+\frac{3}{2}}(\Sigma)} \leq \epsilon$, there exists a unique function $u \in \operatorname{ker}\left(L_{0}, \delta-\right.$ $\left.\frac{1}{2}\right)^{\perp}$ with $\|u\|_{H_{s, \delta-\frac{1}{2}}(\Sigma)} \leq \rho^{\prime}$, such that $u$ is a solution of the maximal surface equation (1.9).

Proof. For the given $(g, k) \in \mathcal{V C}_{s+2, \delta+\frac{1}{2}}(\Sigma)$ with $\theta$ the boost ratio, we can choose a $\rho$-ball $\mathcal{B}_{\rho} \subset H_{s, \delta-\frac{1}{2}}(\Sigma)$, with $\rho$ small enough depending only on $\theta$, $\|g-e\|_{H_{s+2, \delta+\frac{1}{2}}}+\|k\|_{H_{s+1, \delta+\frac{3}{2}}}$ and $\lambda$ as in Proposition 4.4. Then the map $\mathcal{H}$ is continuously differentiable w.r.t. $u$ as a map $\mathcal{B}_{\rho} \cap \operatorname{ker}\left(L_{0}, \delta-\frac{1}{2}\right)^{\perp} \rightarrow$ $H_{s-2, \delta+\frac{3}{2}}(\Sigma)$, and the Fréchet derivative is $\left(D_{u} \mathcal{H}\right)_{(g, k, 0)}=L_{0} v=\left(\triangle_{g}-|k|_{g}^{2}\right) v$ by Lemma 4.9. The coefficient of $L_{0}$ satisfies the hypothesis (4.9), where $s_{0}=s+1$ and $\delta_{0}=\delta+\frac{1}{2}$ by the multiplication Lemma 2.5, the elliptic constant equals to $\lambda$ and $\left\|a_{0,2}-e\right\|_{H_{s+2, \delta+\frac{1}{2}}(\Sigma)},\left\|a_{0,1}\right\|_{H_{s+1, \delta+\frac{3}{2}}(\Sigma)},\left\|a_{0,0}\right\|_{H_{s, \delta+\frac{5}{2}}(\Sigma)}$ are bounded from above by a constant depending only on $\|g-e\|_{H_{s+2, \delta+\frac{1}{2}}(\Sigma)}$ and $\|k\|_{H_{s+1, \delta+\frac{3}{2}}(\Sigma)}$. So $\left(D_{u} \mathcal{H}\right)_{(g, k, 0)}$ is an isomorphism $\operatorname{ker}\left(L_{0}, \delta-\frac{1}{2}\right)^{\perp} \rightarrow$ $H_{s-2, \delta+\frac{3}{2}}(\Sigma)$ by Lemma 4.7 , since $a_{0,0}=-|k|_{g}^{2} \leq 0$. Now we will show that the conditions in the Quantitative Inverse Function Theorem 4.10 are satisfied. By Lemma 4.8, there exists a constant $C_{0}$ depending only on $\lambda$, $\left\|a_{0,2}-e\right\|_{H_{s+2, \delta+\frac{1}{2}}(\Sigma)},\left\|a_{0,1}\right\|_{H_{s+1, \delta+\frac{3}{2}}(\Sigma)},\left\|a_{0,0}\right\|_{H_{s, \delta+\frac{5}{2}}(\Sigma)}$, such that,

$$
\left\|L_{0}^{-1}\right\|_{L\left(H_{s-2, \delta+\frac{3}{2}}(\Sigma), \operatorname{ker}\left(L_{0}, \delta-\frac{1}{2}\right)^{\perp}\right)} \leq C_{0} .
$$

Abbreviate the operator norm $\|\cdot\|_{L\left(H_{s, \delta-\frac{1}{2}}(\Sigma), H_{s-2, \delta+\frac{3}{2}}(\Sigma)\right)}=\|\cdot\|$. Let us study $\left\|D_{u} \mathcal{H}(g, k, u)-D_{u} \mathcal{H}(g, k, 0)\right\|$. Fix the boost evolution $\left(\Omega_{\theta}, \gamma\right)$ of $(g, k)$, with $\|\gamma-\tilde{\eta}\|_{H_{s+2, \delta}\left(\Omega_{\theta}\right)}$ uniformly bounded by a constant depending only on $\lambda$ and $\|g-e\|_{H_{s+2, \delta+\frac{1}{2}}(\Sigma)}+\|k\|_{H_{s+1, \delta+\frac{3}{2}}(\Sigma)}$. Then $D_{u} \mathcal{H}(g, k, u)$ is the first variation $D_{u}\left(H_{u}\right)$ of $H_{u}$ w.r.t. $u$ inside $\left(\Omega_{\theta}, \gamma\right)$. From the formula of $H_{u}$ in (4.8), we know that $D_{u}\left(H_{u}\right)$ is a second order differential operator. The coefficients of $D_{u}\left(H_{u}\right)$ are constituted by algebraic expressions of $\partial u, \partial^{2} u$ and components of $\gamma, \partial \gamma, \partial^{2} \gamma$ evaluated at $(x, u(x))$. Let $a$ be any component of $\partial^{2} \gamma$ (similar 
for $\gamma$ and $\partial \gamma$ ), using the Newton-Leibniz formula,

$$
a(x, u(x))-a(x, 0)=\left(\int_{\tau=0}^{1} \partial_{t} a(x, \tau u(x)) d \tau\right) u(x)
$$

where $\partial_{t} a(x, u(x))$ has uniform $H_{s-2, \delta+\frac{7}{2}}(\Sigma)$ norm depending only on $\left\|\partial^{3} \gamma\right\|_{H_{s-1, \delta+3}\left(\Omega_{\theta}\right)}$ and $\rho$ by Lemma 4.2. So $\|a(x, u(x))-a(x, 0)\|_{H_{s-2, \delta+\frac{5}{2}}(\Sigma)} \leq$ $C_{3}\|u\|_{H_{s, \delta-\frac{1}{2}}(\Sigma)}$ by the multiplication Lemma 2.6, where $C_{3}$ depends only on $\|\gamma-\tilde{\eta}\|_{H_{s+2, \delta}\left(\Omega_{\theta}\right)}$ and $\rho$. Hence by comparing the coefficients of $D_{u} \mathcal{H}(g, k, u)$ with $D_{u} \mathcal{H}(g, k, 0)$, we can choose $\|u\|_{H_{s, \delta-\frac{1}{2}}(\Sigma)} \leq \rho^{\prime}$ with $\rho^{\prime}$ small enough, depending only on $\|\gamma-\tilde{\eta}\|_{H_{s+2, \delta}\left(\Omega_{\theta}\right)}$ and $C_{0}$, such that,

$$
\left\|D_{u} \mathcal{H}(g, k, u)-D_{u} \mathcal{H}(g, k, 0)\right\| \leq \frac{1}{2 C_{0}}
$$

For the $\rho^{\prime}$ chosen above, if we take $\epsilon<\frac{\rho^{\prime}}{2 C_{0}}$, then

$$
\|0-\mathcal{H}(g, k, 0)\|_{H_{s-2, \delta+\frac{3}{2}}(\Sigma)}=\left\|t r_{g} k\right\|_{H_{s-2, \delta+\frac{3}{2}}(\Sigma)}<\frac{\rho^{\prime}}{2 C_{0}} .
$$

Now by the Quantitative Inverse Function Theorem 4.10, if we choose the $\epsilon$ and $\rho^{\prime}$ as above, where $\epsilon$ and $\rho^{\prime}$ depend only on $\lambda,\|g-e\|_{H_{s+2, \delta+\frac{1}{2}}(\Sigma)}$ and $\|k\|_{H_{s+1, \delta+\frac{3}{2}}(\Sigma)}$, there exists a unique $u \in \mathcal{B}_{\rho^{\prime}} \cap \operatorname{ker}\left(L_{0}, \delta-\frac{1}{2}\right)^{\perp}$, such that $u$ solves $\mathcal{H}(u)=0$.

\subsection{Proof of the main theorem}

Here we will study the properties of the maximal graph gotten above. We will improve the regularity of the solution using a bootstrap argument, and show that the ADM mass of the maximal graph is the same as that of the given data. Moreover the maximal graph is axisymmetric if $(g, k)$ is axisymmetric, and the angular momentum of the maximal graph is the same as that of $(g, k)$.

In Theorem 4.12, the solution $u$ has only $s$ weak derivatives due to the Contraction Mapping Principle. In fact, by exploring the structure of the mean curvature operator (4.8), we can gain more regularity for $u$. 
Lemma 4.13 (Regularity analysis). In Theorem 4.12, the solution $u \in$ $H_{s+2, \delta-\frac{1}{2}}(\Sigma)$. Denote $M=$ Graph $_{u}$, and let $g_{M}$ be the metric and $k_{M}$ the second fundamental form induced by the embedding $M \subset(\mathcal{V}, \gamma)$, then $\left(g_{M}, k_{M}\right) \in \mathcal{V} \mathcal{C}_{s+1, \delta+\frac{1}{2}}(\Sigma)$.

Proof. In the local coordinates formula (4.8), we can collect together all the terms containing $\partial_{i j}^{2} u$, then the maximal surface equation $\mathcal{H}(u)=0$ can be rewritten as a linear second order elliptic equation for $u$ with $\partial u$ and $u$ terms as coefficients:

$$
\left(g^{M}\right)^{i j}(x, u(x)) u_{i j}=f(x),
$$

where $f(x)$ is a polynomial of $g^{M}(x, u(x)), \partial u, \gamma(x, u(x))$ and $(\partial \gamma)(x, u(x))$. First the spacelike property of $M=G_{r a p h}$ implies that $\left(g^{M}\right)^{i j}$ is elliptic. Furthermore, $\left(g^{M}\right)^{i j}(x, u(x))-e^{i j}(x)=\gamma^{i j}-e^{i j}+\frac{\nu^{2}}{\alpha^{2}}\left(\beta^{i}-\alpha U^{i}\right)\left(\beta^{j}-\alpha U^{j}\right)$ $\in H_{s-1, \delta+\frac{1}{2}}(\Sigma), f(x) \in H_{s-1, \delta+\frac{3}{2}}(\Sigma)$ by the argument in the proof of Proposition 4.4, Lemma 4.2, and the Banach algebra property in Lemma 2.6. Since $\left(g^{M}\right)^{i j}$ lie in $C^{0}$ and $H_{s-1}$ locally, $u \in\left(H_{s+1}\right)_{l o c}(\Sigma)$ by standard elliptic regularity theory [18]. Furthermore, the linear operator $L u=\left(g^{M}\right)^{i j} \partial_{i j}^{2} u$ satisfies the hypothesis of the weighted elliptic regularity [7, Theorem 6.1] since $s \geq 4$, hence $u \in H_{s+1, \delta-\frac{1}{2}}(\Sigma)$ by [7, Theorem 6.1]. Now we can bootstrap this process. In fact, by the composition Lemma 4.2, the right-hand side $f(x)$ lies in at most $H_{s, \delta+\frac{3}{2}}(\Sigma)$ since there are $\partial \gamma(x, u(x))$ terms. So bootstrap ends when $u \in H_{s+2, \delta-\frac{1}{2}}(\Sigma)$.

On the graph $M,\left(g_{M}\right)_{i j}=\left(g_{i j}^{2}+\beta_{i} u_{j}+\beta_{j} u_{i}-\left(\alpha^{2}-\beta^{2}\right) u_{i} u_{j}\right)(x, u(x))$ by $(2.14)$,

$$
\begin{aligned}
\left(k_{M}\right)_{i j}= & \nu \cdot\left\{\left(\partial_{i}+u_{i} \partial_{t}\right)\left(U^{\mu}+T^{\mu}\right) \cdot\left(\gamma_{\mu j}+u_{j} \gamma_{\mu t}\right)\right. \\
& \left.+\left(U^{\mu}+T^{\mu}\right)\left(\Gamma_{i \mu, j}+u_{i} \Gamma_{t \mu, j}+u_{j} \Gamma_{i \mu, t}+u_{i} u_{j} \Gamma_{t \mu, t}\right)\right\}
\end{aligned}
$$

by formula (4.8). So by the proof of Proposition 4.4, $\left(\left(g_{M}\right)_{i j}-e_{i j}\right) \in$ $H_{s+1, \delta+\frac{1}{2}}(\Sigma)$ and $\left(k_{M}\right)_{i j} \in H_{s, \delta+\frac{3}{2}}(\Sigma)$.

In order to define the ADM mass and linear momentum, we need to assume $-\frac{3}{2}<\delta<-1$, then by the embedding Lemma 2.5, $\left(g_{M}-e\right) \in$ $C_{\kappa}^{s-1}(\Sigma)$ and $k_{M} \in C_{\kappa+1}^{s-2}(\Sigma)$ for some $\frac{1}{2}<\kappa<1$, which satisfy the conditions (1.5). Similar conditions are also satisfied by $(g-e, k)$. We can defined the ADM mass $m, m_{M}$ for $(g, k)$ and $\left(g_{M}, k_{M}\right)$, respectively.

Lemma 4.14. For $-\frac{3}{2}<\delta<-1$, in Theorem 4.12, $m=m_{M}$.

Proof. We will use the multiplication Lemma 2.5 frequently when we multiply two Sobolev functions. Now $\left(g_{M}\right)_{i j}(x)-g_{i j}(x, u(x))=\left(\beta_{i} u_{j}+\beta_{j} u_{i}-\right.$ 
$\left.\left(\alpha^{2}-\beta^{2}\right) u_{i} u_{j}\right)(x, u(x)) \quad$ by $(2.14) . \quad \beta(x, u(x)) \in H_{s+1, \delta+\frac{1}{2}}(\Sigma)$ and $\partial u \in$ $H_{s+1, \delta+\frac{1}{2}}(\Sigma)$ imply $\left(g_{M}\right)_{i j}(x)-g_{i j}(x, u(x)) \in H_{s+1, \delta+1}(\Sigma)$.

$$
g_{i j}(x, u(x))-g_{i j}(x)=\left\{\int_{s=0}^{1} \partial_{t} g_{i j}(x, s u(x)) d s\right\} \cdot u(x),
$$

which shows $\left\{g_{i j}(x, u(x))-g_{i j}(x)\right\} \in H_{s+1, \delta+1}(\Sigma)$, since $\partial_{t} g_{i j}(x, s u(x)) \in$ $H_{s+1, \delta+\frac{3}{2}}(\Sigma) \quad$ and $u \in H_{s+2, \delta-\frac{1}{2}}(\Sigma)$. Hence $\left\{\left(g_{M}\right)_{i j}(x)-g_{i j}(x)\right\} \in$ $H_{s+1, \delta+1}(\Sigma) \subset C_{\kappa}^{s-1}(\Sigma)$, for some $1<\kappa<\delta+\frac{5}{2}$ by the embedding Lemma 2.5. By checking the definition (1.6), we know that a error term of decay rate $o\left(r^{-1}\right)$ will not change the mass, so $m=m_{M}$.

Now we will study the preservation of symmetry by this construction. We need a lemma about symmetry preserving by the reduced EVE (3.3).

Lemma 4.15. Given a vacuum data $(g, k) \in \mathcal{V C}_{s+2, \delta+\frac{1}{2}}(\Sigma)$, and $\left(\Omega_{\theta}, \gamma\right)$ the boost evolution of $(g, k)$ given by Theorem 3.7. Suppose that both $(g, k)$ and $e$ are symmetric under a Killing vector field $\xi$ on $\Sigma$, i.e., $(g, k)$ satisfy (1.7), and $\mathcal{L}_{\xi} e=0$, where $e$ is the canonical metric on $\Sigma$. Then the parallel translation $\tilde{\xi}$ of $\xi$ into $\Omega_{\theta}$ is a Killing vector field of $\gamma$.

Proof. Now let $\phi_{s}: \Sigma \rightarrow \Sigma$ be the one parameter group of diffeomorphisms corresponding to $\xi$. Then $\left(\phi_{s}\right)^{*} g=g,\left(\phi_{s}\right)^{*} k=k$ and $\left(\phi_{s}\right)^{*} e=e$. Now let us extend $\phi_{s}$ to a diffeomorphism $\tilde{\phi}_{s}: \Omega_{\theta} \rightarrow \Omega_{\theta}$ by

$$
\tilde{\phi}_{s}(x, t)=\left(\phi_{s}(x), t\right) .
$$

Then $\left(\tilde{\phi}_{s}\right)^{*} \tilde{e}=\tilde{e}$ where $\tilde{e}$ is defined by (2.1). By the initial conditions (3.5) and (3.6) for $\gamma$, we know that $\gamma_{s}=\left(\tilde{\phi}_{s}\right)^{*} \gamma$ has the same initial conditions as those of $\gamma$ on $\Sigma$. If we can show that $\gamma_{s}$ also solves the reduced (EVE) (3.3), the uniqueness in Theorem 3.7 implies that $\gamma_{s}=\gamma$. Since $\gamma_{s}$ is Ricci flat, we only need to show that $\left(\Omega_{\theta}, \gamma_{s}\right)$ is also in a harmonic gauge, or equivalently, $i d:\left(\Omega_{\theta}, \gamma_{s}\right) \rightarrow\left(\Omega_{\theta}, \tilde{e}\right)$ is a wave map. By pulling back the wave map equation $\square_{(\gamma, \tilde{e})} i d=0$ by $\tilde{\phi}$, we get $\square_{\left(\left(\tilde{\phi}_{s}\right)^{*} \gamma_{i}\left(\tilde{\phi}_{s}\right)^{*} \tilde{e}\right)} i d=0$, which reduces to $\square_{\left(\gamma_{s}, \tilde{e}\right)} i d=0$. This means that $\gamma_{s}$ is also in a harmonic gauge, hence $\gamma_{s}=$ $\gamma$. Now the vector field corresponding to $\tilde{\phi}_{s}$ is clearly the parallel translation of $\xi$ into $\Omega_{\theta}$.

Now we can prove the preservation of symmetry for the maximal surface. 
Theorem 4.16. Given $s \geq 4,-2<\delta<-1$. Suppose $(\Sigma, e)$ is a 3-manifold, which is Euclidean at infinity and axisymmetric in the sense of Definition 1.3. If $(g, k) \in \mathcal{V} \mathcal{C}_{s+2, \delta+\frac{1}{2}}^{a}(\Sigma)$ is axisymmetric, and $\left\|t r_{g} k\right\|_{H_{s-2, \delta+\frac{3}{2}}}(\Sigma) \leq \epsilon$ with $\epsilon$ given by Theorem 4.12, then the solution $u$ of the maximal surface equation (1.9) given in Theorem 4.12 is axisymmetric, i.e., $\partial_{\varphi} u=0$. Hence $\left(\Sigma, g_{u}, k_{u}\right)$ is axisymmetric, and the angular momentum of $\left(g_{u}, k_{u}\right)$ equals that of $(g, k)$.

Proof. By Theorem 4.12, $\mathcal{H}(g, k, u)=0$ has a unique solution $u \in \mathcal{B}_{\rho^{\prime}} \cap$ $\operatorname{ker}\left(L_{0}, \delta-\frac{1}{2}\right)^{\perp}$. Let $\phi_{s}$ be the diffeomorphism corresponding to the Killing vector field $\xi=\frac{\partial}{\partial \varphi}$ in Definition 1.3, and $\tilde{\phi}_{s}$ the extension given in (4.27). When $(g, k)$ is also axisymmetric, the boost solution $\left(\Omega_{\theta}, \gamma\right)$ is invariant under $\tilde{\phi}_{s}$ by Lemma 4.15. Now pulling back $\mathcal{H}(g, k, u)=0$ by $\tilde{\phi}_{s}$, we can see that $\phi_{s}^{*} u$ is a solution of $\mathcal{H}\left(\phi_{s}^{*} g, \phi_{s}^{*} k, \phi_{s}^{*} u\right)=0$, hence $\mathcal{H}\left(g, k, \phi_{s}^{*} u\right)=0$. Since $(\Sigma, e)$ and $(g, k)$ are all invariant under $\phi_{s}, \operatorname{ker}\left(L_{0}, \delta-\frac{1}{2}\right)$ and hence $\operatorname{ker}\left(L_{0}, \delta-\frac{1}{2}\right)^{\perp}$ are also invariant under $\phi_{s}$, which means that $\left(\phi_{s}\right)^{*} u \in \mathcal{B}_{\rho^{\prime}} \cap$ $\operatorname{ker}\left(L_{0}, \delta-\frac{1}{2}\right)^{\perp}$, then uniqueness in Theorem 4.12 implies that $\left(\phi_{s}\right)^{*} u=u$. So $u$ is axisymmetric, hence is $\left(g_{u}, k_{u}\right)$ since $\gamma$ is also axisymmetric.

For the angular momentum, we have another formula, which is called Komar integral (for angular momentum) (see [24, Section 11.2] for definition and equivalence with (1.8)),

$$
J(S)=\frac{1}{16 \pi} \int_{S} * d \xi,
$$

where $*$ is the Hodge star operator w.r.t. $\gamma$, and $\xi$ the killing vector field. Since $* d \xi$ is a closed form, we know that $J(S)$ is invariant for any two spacelike close surface $S$ and $S^{\prime}$ which are homologous to each other. To show that $(\Sigma, g, k)$ and $\left(\operatorname{Graph}_{u}, g_{u}, k_{u}\right)$ have the same angular momentum, we can take a coordinate two surface $S_{0}=\partial B_{R}(0)$ inside $\Sigma$ (recall that $\left.\Sigma \simeq \mathbb{R}^{3} \backslash\{0\}\right)$, then the angular momentum of $\Sigma$ is $J\left(S_{0}\right)$. As the boost evolution spacetime $\Omega$ is a subset of $\Sigma \times \mathbb{R}$, let $S_{0}^{\prime}$ be the intersection of the cylinder $S_{0} \times \mathbb{R}$ with $\operatorname{Graph}_{u}$ inside $(\Omega, \gamma)$, then $S_{0}^{\prime}$ is a smooth two surface when $R$ is large enough. Clearly $S_{0}^{\prime}$ is homologous to $S_{0}$, hence $J\left(S_{0}^{\prime}\right)=J\left(S_{0}\right)$. The angular momentum of $\left(\operatorname{Graph}_{u}, g_{u}, k_{u}\right)$ is $J\left(S_{0}^{\prime}\right)$ which equals that of $(\Sigma, g, k)$.

\section{Acknowledgments}

The author would like to express his gratitude to his advisor Professor Richard Schoen for all of his helpful guidance and constant encouragement. 
He would like to thank Professor Rafe Mazzeo and Professor Leon Simon for lots of useful talks. He would also like to thank his friend Pin Yu for talking a lot about the hyperbolic equations. Finally, the author thanks the referee for comments.

\section{A. Appendix}

\section{A.1. Geometry of hypersurface}

Here we show the detailed calculation of the mean curvature of a level surface. Part of the results here already appeared in [1]. First let us calculate the future-directed timelike unit normal vector of $\Sigma_{t}$ defined by $T=-\frac{\nabla t}{\mid \nabla t}$, which is given by:

$$
T=-\alpha \nabla t=-\alpha\left(\gamma^{t t} \partial_{t}+\gamma^{t i} \partial_{i}\right)=\alpha^{-1}\left(\partial_{t}-\beta\right) .
$$

$\operatorname{Graph}_{u}$ can be viewed as the level surface of $(u-t)=0$, so the unit normal of Graph $_{u}$ is $N=\frac{\nabla(u-t)}{|\nabla(u-t)|}$. Now

$$
\begin{aligned}
\nabla u & =\gamma^{t j} u_{j} \partial_{t}+\gamma^{i j} u_{j} \partial_{i}=\frac{1}{\alpha^{2}}\langle\beta, D u\rangle \partial_{t}+D u-\frac{1}{\alpha^{2}}\langle\beta, D u\rangle \beta^{i} \partial_{i} \\
& =\frac{1}{\alpha}\langle\beta, D u\rangle T+D u
\end{aligned}
$$

So $N$ is calculated as

$$
\begin{aligned}
\nabla(u-t) & =\frac{1}{\alpha^{2}}\langle\beta, D u\rangle \partial_{t}+D u-\frac{1}{\alpha^{2}}\langle\beta, D u\rangle \beta^{i} \partial_{i}+\frac{1}{\alpha^{2}} \partial_{t}-\frac{1}{\alpha^{2}} \beta^{i} \partial_{i} \\
& =D u+\frac{1+\langle\beta, D u\rangle}{\alpha^{2}}\left(\partial_{t}-\beta\right) \\
& =\alpha^{-1}(1+\langle\beta, D u\rangle)\left(\frac{\alpha D u}{1+\langle\beta, D u\rangle}+T\right) .
\end{aligned}
$$

Writing $U=\frac{\alpha D u}{1+\langle\beta, D u\rangle}$, then $N=\frac{U+T}{|U+T|}$, where $|U+T|=\left(1-|U|^{2}\right)^{1 / 2}$, so we get (2.11).

Denoting $M=G_{r a p h}$, let us calculate the mean curvature. For completeness we give the inverse metric matrix $\left(g_{M}\right)^{-1}$ of $g_{M}$ in (2.14). First we need to calculate the co-frame of (2.13). Denoting them by $\alpha^{i}=a_{k}^{i} d x^{k}+$ $a_{t}^{i} d t: i, k=1,2,3$, then they should satisfy:

$$
\alpha^{i}\left(\alpha_{k}\right)=\delta_{k}^{i}, \quad \alpha^{i}(N)=0 .
$$


The last equation gives

(A.5)

$$
\begin{aligned}
\left(a_{k}^{i} d x^{k}+a_{t}^{i} d t\right)[\alpha(U+T)] & =\left(a_{k}^{i} d x^{k}+a_{t}^{i} d t\right)\left(\frac{\alpha^{2} D u}{1+\langle\beta, D u\rangle}+\left(\partial_{t}-\beta\right)\right) \\
& =a_{k}^{i}\left(\frac{\alpha^{2} u^{k}}{1+\langle\beta, D u\rangle}-\beta^{k}\right)+a_{t}^{i}=0 .
\end{aligned}
$$

So

$$
a_{t}^{i}=a_{k}^{i}\left(\beta-\frac{\alpha^{2} D u}{1+\langle\beta, D u\rangle}\right)^{k}=a_{k}^{i}(\beta-\alpha U)^{k} .
$$

Putting into the first one in (A.4), we have

$$
\text { (A.7) } \quad\left(a_{k}^{i} d x^{k}+a_{l}^{i}\left(\beta^{l}-\alpha U^{l}\right) d t\right)\left(\partial_{k}+u_{k} \partial_{t}\right)=a_{k}^{i}+a_{l}^{i}\left(\beta^{l}-\alpha U^{l}\right) u_{k}=\delta_{k}^{i} .
$$

Denoting matrix $A=\left(a_{k}^{i}\right)$, then the above equations change to the matrix equation

$$
A \cdot\left[i d+(\beta-\alpha U)(D u)^{t}\right]=i d
$$

Solving the last matrix equation ${ }^{22}$, we get

$$
\begin{aligned}
a_{k}^{i} & =I d-\frac{\left(\beta^{i}-\alpha U^{i}\right) u_{k}}{1+\langle\beta-\alpha U, D u\rangle}=I d-\frac{\left(\beta^{i}-\alpha U^{i}\right) u_{k}}{1+\langle\beta, D u\rangle-(1+\langle\beta, D u\rangle)|U|^{2}} \\
& =I d-\nu^{2} \frac{\left(\beta^{i}-\alpha U^{i}\right) u_{k}}{1+\langle\beta, D u\rangle}=I d-\nu^{2}(\beta / \alpha-U)^{i} U_{k},
\end{aligned}
$$

where we have used $U=\frac{\alpha D u}{1+\langle\beta, D u\rangle}$, and $\nu^{-2}=1-|U|^{2}$. Then

$$
\begin{aligned}
a_{t}^{i} & =a_{k}^{i}(\beta-\alpha U)^{k}=\left(\delta_{k}^{i}-\nu^{2} \frac{\left(\beta^{i}-\alpha U^{i}\right) u_{k}}{1+\langle\beta, D u\rangle}\right)\left(\beta^{k}-\alpha U^{k}\right) \\
& =\beta^{i}-\nu^{2} \frac{\left(\beta^{i}-\alpha U^{i}\right)\langle\beta, D u\rangle}{1+\langle\beta, D u\rangle}-\alpha U^{i}+\nu^{2}\left(\beta^{i}-\alpha U^{i}\right)|U|^{2} \\
& =\left(1+\nu^{2}|U|^{2}\right)\left(\beta^{i}-\alpha U^{i}\right)-\nu^{2} \frac{\langle\beta, D u\rangle}{1+\langle\beta, D u\rangle}\left(\beta^{i}-\alpha U^{i}\right)
\end{aligned}
$$

\footnotetext{
${ }^{22}$ The inverse of $I d+\mathbf{u v}^{t}$ is given by $I d-\frac{\mathbf{u v}}{1+\mathbf{u} \cdot \mathbf{v}}$.
} 


$$
\begin{aligned}
& =\nu^{2}\left(\beta^{i}-\alpha U^{i}\right)-\nu^{2} \frac{\langle\beta, D u\rangle}{1+\langle\beta, D u\rangle}\left(\beta^{i}-\alpha U^{i}\right) \\
& =\frac{\nu^{2}}{1+\langle\beta, D u\rangle}(\beta-\alpha U)^{i} .
\end{aligned}
$$

So the co-frame is given by

$$
\alpha^{i}=\left(\delta_{k}^{i}-\nu^{2}(\beta / \alpha-U)^{i} U_{k}\right) d x^{k}+\frac{\nu^{2}}{1+\langle\beta, D u\rangle}(\beta-\alpha U)^{i} d t
$$

Taking inner product of the co-frame with respect to $\gamma^{-1}$, we can calculate $g_{M}^{-1}$.

$$
\begin{aligned}
\left(g_{M}\right)^{i j}= & \left\langle\left(\delta_{k}^{i}-\nu^{2}(\beta / \alpha-U)^{i} U_{k}\right) d x^{k}+\frac{\nu^{2}(\beta-\alpha U)^{i}}{1+\langle\beta, D u\rangle} d t\right. \\
& \left.\left(\delta_{l}^{j}-\nu^{2}(\beta / \alpha-U)^{j} U_{l}\right) d x^{l}+\frac{\nu^{2}(\beta-\alpha U)^{j}}{1+\langle\beta, D u\rangle} d t\right\rangle_{\gamma} \\
= & \left(\delta_{k}^{i}-\nu^{2}(\beta / \alpha-U)^{i} U_{k}\right)\left(\delta_{l}^{j}-\nu^{2}(\beta / \alpha-U)^{j} U_{l}\right)\left(g^{k l}-\frac{1}{\alpha^{2}} \beta^{k} \beta^{l}\right) \\
& +\left(\delta_{k}^{i}-\nu^{2}(\beta / \alpha-U)^{i} U_{k}\right) \frac{\nu^{2}(\beta-\alpha U)^{j}}{1+\langle\beta, D u\rangle} \frac{\beta^{k}}{\alpha^{2}} \\
& +\left(\delta_{k}^{j}-\nu^{2}(\beta / \alpha-U)^{j} U_{k}\right) \frac{\nu^{2}(\beta-\alpha U)^{i}}{1+\langle\beta, D u\rangle} \frac{\beta^{k}}{\alpha^{2}} \\
& -\frac{1}{\alpha^{2}} \frac{\nu^{4}(\beta-\alpha U)^{i}(\beta-\alpha U)^{j}}{(1+\langle\beta, D u\rangle)^{2}} \\
= & g^{i j}-\frac{1}{\alpha^{2}} \beta^{i} \beta^{j}-\nu^{2}(\beta / \alpha-U)^{j} U^{i}-\nu^{2}(\beta / \alpha-U)^{i} U^{j} \\
& +\frac{\nu^{2}(\beta-\alpha U)^{j} \beta^{i}\langle\beta, D u\rangle}{\alpha^{2}(1+\langle\beta, D u\rangle)}+\frac{\nu^{2}(\beta-\alpha U)^{i} \beta^{j}\langle\beta, D u\rangle}{\alpha^{2}(1+\langle\beta, D u\rangle)} \\
& +\nu^{4}(\beta / \alpha-U)^{i}(\beta / \alpha-U)^{j}|U|^{2}-\frac{\nu^{4}(\beta-\alpha U)^{i}(\beta-\alpha U)^{j}}{\alpha^{2}(1+\langle\beta, D u\rangle)^{2}}\langle\beta, D\rangle^{2} \\
& +\frac{\nu^{2}}{\alpha^{2}} \frac{\beta^{i}(\beta-\alpha U)^{j}+\beta \beta^{j}(\beta-\alpha U)^{i}}{1+\langle\beta, D u\rangle} \\
& -2 \frac{\nu^{4}(\beta-\alpha U)^{i}(\beta-\alpha U)^{j}}{\alpha^{2}(1+\langle\beta, D u\rangle)^{2}}\langle\beta, D u\rangle-\frac{\nu^{4}(\beta-\alpha U)^{i}(\beta-\alpha U)^{j}}{\alpha^{2}(1+\langle\beta, D u\rangle)^{2}}
\end{aligned}
$$




$$
\begin{aligned}
= & g^{i j}-\frac{1}{\alpha^{2}} \beta^{i} \beta^{j}-\nu^{2}(\beta / \alpha-U)^{j} U^{i}-\nu^{2}(\beta / \alpha-U)^{i} U^{j} \\
& +\nu^{4}(\beta / \alpha-U)^{i}(\beta / \alpha-U)^{j}|U|^{2}+\frac{\nu^{2}}{\alpha^{2}}\left[\beta^{i}(\beta-\alpha U)^{j}+\beta^{j}(\beta-\alpha U)^{i}\right] \\
& -\frac{\nu^{4}}{\alpha^{2}}(\beta-\alpha U)^{i}(\beta-\alpha U)^{j} \\
= & g^{i j}-\frac{1}{\alpha^{2}} \beta^{i} \beta^{j}+\frac{\nu^{2}}{\alpha^{2}}(\beta-\alpha U)^{i}(\beta-\alpha U)^{j} .
\end{aligned}
$$

\section{A.2. Linear boost estimates on an end}

Here we will give a detailed version of linear boost estimates on an Euclidean end using method in $[8,10]$. It was also mentioned in [3]. We will mainly give the energy estimates needed to prove Theorem 3.6. For convenience, we sometime abbreviate $V_{\theta, \lambda}=V$ in this section. Given a regularly hyperbolic metric $\gamma^{\mu \nu}$ and a $\mathbb{R}^{N}$-valued function $u$ in $V_{\theta, \lambda}$, we can associate it with the energy-momentum tensor $T^{\mu \nu 23}$ :

$$
T^{\mu \nu}=G^{\mu \nu \rho \sigma} D_{\rho} u \cdot D_{\sigma} u,{ }^{24}
$$

where

$$
G^{\mu \nu \rho \sigma}=\gamma^{\mu \rho} \gamma^{\nu \sigma}+\gamma^{\mu \sigma} \gamma^{\nu \rho}-\gamma^{\mu \nu} \gamma^{\rho \sigma}
$$

Given the unit normal $\tilde{n}$ of $\left\{E_{\tau}\right\}$ defined in (3.12), the momentum vector field relative to $\tilde{n}$ is

$$
P^{\mu}=T^{\mu \nu} \tilde{n}_{\nu}
$$

Furthermore, the divergence of $P^{\mu}$ is

$$
D_{\mu} P^{\mu}=2\left(\gamma^{\rho \sigma} \tilde{n}_{\rho} D_{\sigma} u\right) \cdot \gamma^{\mu \nu} D_{\mu \nu}^{2} u+Q
$$

where

$$
Q=\Lambda^{\mu \nu} D_{\mu} u \cdot D_{\nu} u, \text { with } \Lambda^{\mu \nu}=D_{\rho}\left(G^{\mu \nu \rho \sigma} \tilde{n}_{\sigma}\right) .
$$

Let $N^{-2}=-\langle D \tau, D \tau\rangle_{\gamma}$ be the lapse function for $\tau$ w.r.t. $\gamma$ and $n=N D \tau$ the unit co-normal of $\left\{E_{\tau}\right\}$ w.r.t. $\gamma$. We introduce an orthonormal frame $\left\{e_{0}, e_{1}, \cdots, e_{n-1}\right\}$ w.r.t. $\gamma$, such that $e_{0}$ is along the direction of $\tilde{n}^{\mu}=\gamma^{\mu \nu} \tilde{n}_{\mu}$, i.e., $e_{0}=\frac{N}{\tilde{N}} \tilde{n}^{\mu}$, where $\left(\frac{N}{\tilde{N}}\right)^{-2}=|\tilde{n}|_{\gamma}^{2}$, and $e_{i}$ is perpendicular to $\tilde{n}^{\mu}$. According

${ }^{23}$ See [8, Equation (4.6)].

${ }^{24}$ Here the inner product of $D_{\rho} u \cdot D_{\sigma} u=\sum_{k=1}^{N} D_{\rho} u^{k} D_{\sigma} u^{k}$. 
to $\left[8\right.$, Section 2], we know that $|\tilde{n}|_{\gamma}^{2}=\gamma^{\mu \nu} \tilde{n}_{\mu} \tilde{n}_{\nu}=\left(\frac{N}{\tilde{N}}\right)^{-2}$ is bounded from both above and below by some constants depending only on $\theta$ and $h$.

Lemma A.1. When $\gamma$ is regularly hyperbolic, $P^{\mu}$ is past time-like w.r.t. $\gamma$. Proof. $T^{\mu \nu}=2 D^{\mu} u \cdot D^{\nu} u-|D u|_{\gamma}^{2} \gamma^{\mu \nu}$, so $\quad P^{\mu}=T^{\mu \nu} \tilde{n}_{\nu}=2 D^{\mu} u \cdot D^{\nu} u \tilde{n}_{\nu}-$ $|D u|_{\gamma}^{2} \tilde{n}^{\mu}$, and

$$
\begin{aligned}
\gamma_{\mu \nu} P^{\mu} P^{\nu}= & 4 \gamma_{\mu \nu}\left(D^{\mu} u \cdot D^{\rho} u \tilde{n}_{\rho}\right)\left(D^{\nu} u \cdot D^{\sigma} u \tilde{n}_{\sigma}\right) \\
& -4|D u|_{\gamma}^{2}\left(D^{\mu} u \tilde{n}_{\mu} \cdot D^{\nu} u \tilde{n}_{\nu}\right)+|D u|_{\gamma}^{4}|\tilde{n}|_{\gamma}^{2} \\
\leq & |D u|_{\gamma}^{4}|\tilde{n}|_{\gamma}^{2} \leq 0 .
\end{aligned}
$$

The first " $\leq$ " comes from Cauchy-Schwartz inequality, and the second comes from the fact that $\tilde{n}$ is time-like w.r.t. $\gamma$.

Take $l^{\mu}$ as a future timelike vector field, then in the orthonormal frame $\left\{e_{0}, e_{1}, \ldots, e_{n-1}\right\}$ as above, $l^{0}>\sqrt{\sum_{i=1}^{n-1}\left(l^{i}\right)^{2}}$, and

$$
\begin{aligned}
\gamma_{\mu \nu} P^{\mu} l^{\mu}= & 2\left[\left(D_{0} u\right) l^{0}+\left(D_{i} u\right) l^{i}\right]\left(D_{0} u\right)(\tilde{N} / N)-\left[-\left(D_{0} u\right)^{2}\right. \\
& \left.+\sum\left(D_{i} u\right)^{2}\right]\left(-l_{0}\right)(\tilde{N} / N) \\
= & {\left[\left(D_{0} u\right)^{2}+\sum\left(D_{i} u\right)^{2}\right] l^{0}(\tilde{N} / N)+2 D_{i} u D_{0} u l^{i}(\tilde{N} / N) } \\
\geq & (\tilde{N} / N)\left[\left(D_{0} u\right)^{2}+\sum\left(D_{i} u\right)^{2}\right]\left(l^{0}-\sqrt{\sum\left(l^{i}\right)^{2}}\right) \geq 0 .
\end{aligned}
$$

The first " $\geq$ " comes from the Cauchy-Schwartz inequality. So it shows that $P$ is past time-like w.r.t. $\gamma$.

Now we introduce the restriction norm and restriction lemma similar to (2.4) and Lemma 2.8. Given $u \in H_{s, \delta}\left(V_{\theta, \lambda}\right)$, the restriction norm to hypersurface $E_{\tau}$ is defined as:

$$
\|u\|_{H_{s, \delta}\left(E_{\tau}, V_{\theta, \lambda}\right)}=\left(\Sigma_{k=0}^{s}\left\|\left.D_{t}^{k} u\right|_{E_{\tau}}\right\|_{H_{s-k, \delta+k}(E)}^{2}\right)^{1 / 2} .
$$

The following restriction lemma follows similar from [8, Lemma 3.1]:

Lemma A.2 (Restriction). $\forall \tau \in(-\theta, \theta)$, we have the following continuous inclusion:

$$
H_{s+1, \delta}\left(V_{\theta, \lambda}\right) \subset H_{s, \delta+\frac{1}{2}}\left(E_{\tau}, V_{\theta, \lambda}\right),
$$

for every $s \in \mathbb{N}$ and $\delta \in \mathbb{R}$.

Now we have the first energy estimates. 
Lemma A.3 (First Energy Estimates). Assume that $\gamma^{\mu \nu}$ is regularly hyperbolic, and $(\gamma-\eta) \in C^{\infty} \cap C^{1,0}(V), a_{1} \in C^{\infty} \cap C^{0,1}(V)$ and $a_{0} \in C^{\infty} \cap$ $C^{0,2}(V)$. For $L$ defined in (3.14), with $a_{2}=\gamma I d$, every $u \in C_{0}^{\infty}(V)$ satisfies the fundamental energy estimates:

$$
\|u\|_{H_{1, \delta+\frac{1}{2}}\left(E_{\tau}, V\right)} \leq c\left(\|u\|_{H_{1, \delta+\frac{1}{2}}(E, V)}+\|\beta\|_{H_{0, \delta+2}(V)}\right)
$$

where $0 \leq \tau \leq \theta, \beta=L u$, and $c$ is a constant depending only on $\theta$, the coefficient of regular hyperbolicity $h$ (3.13) of $\gamma$, and $\|D \gamma\|_{C^{0,1}}+\left\|a_{1}\right\|_{C^{0,1}}+$ $\left\|a_{2}\right\|_{C^{0,2}}$.

Proof. Let $\tilde{P}^{\mu}=\sigma^{2\left(\delta+\frac{3}{2}\right)} P^{\mu}$. Multiply (A.15) by $\sigma^{2\left(\delta+\frac{3}{2}\right)}$, we get:

$$
D_{\mu} \tilde{P}^{\mu}=2 \sigma^{2\left(\delta+\frac{3}{2}\right)}\left(\gamma^{\rho \sigma} \tilde{n}_{\rho} D_{\sigma} u\right) \cdot \gamma^{\mu \nu} D_{\mu \nu}^{2} u+\tilde{Q}
$$

where

$$
\tilde{Q}=\sigma^{2\left(\delta+\frac{3}{2}\right)} Q^{\prime}
$$

with

$$
Q^{\prime}=Q+2(\delta+3 / 2) x^{i} / \sigma^{2} P^{i} \simeq\left(D \gamma * \gamma+\sigma^{-1} \gamma * \gamma\right) D u * D u
$$

Plug in $L u=\beta$,

$$
D_{\mu} \tilde{P}^{\mu}=\sigma^{2\left(\delta+\frac{3}{2}\right)}\left[2\left(\gamma^{\rho \sigma} \tilde{n}_{\rho} D_{\sigma} u\right) \cdot\left(\beta-a_{1} D u-a_{0} u\right)+Q^{\prime}\right]
$$

Now we integrate on the upper part $V_{\tau, \lambda}^{+}=\left\{x \in V_{\tau, \lambda}: t \geq 0\right\}$ for $\tau \leq \theta$. Since $P$ is compactly supported, the divergence theorem in $\left(V_{\tau, \lambda}^{+}, \eta\right)$ gives,

$$
\begin{gathered}
\int_{E_{\tau}} \tilde{P}^{\mu} \tilde{n}_{\mu} d \Sigma_{\tau}-\int_{E} \tilde{P}^{\mu} \tilde{n}_{\mu} d \Sigma+\int_{L_{\tau, \lambda}^{+}} \tilde{P}^{\mu} \tilde{\nu}_{\mu} d \sigma=\int_{V_{\tau, \lambda}^{+}} D_{\mu} \tilde{P}^{\mu} d x \\
=\int_{V_{\tau, \lambda}^{+}} \sigma^{2\left(\delta+\frac{3}{2}\right)}\left[2\left(\gamma^{\rho \sigma} \tilde{n}_{\rho} D_{\sigma} u\right) \cdot\left(\beta-a_{1} D u-a_{0} u\right)+Q^{\prime}\right] d x
\end{gathered}
$$

where $\tilde{\nu}_{\mu}$ is the unit outer co-normal of the upper lateral boundary $L_{\tau, \lambda}^{+}=$ $L_{\tau, \lambda} \cap V_{\tau, \lambda}^{+}$under $\eta$, which is future timelike w.r.t. $\gamma$ by property (4) of the regular hyperbolicity (3.2). Using the fact that $P$ is past timelike 
(Lemma A.1), we know that

$$
\tilde{P}^{\mu} \tilde{\nu}_{\mu}=\sigma^{2\left(\delta+\frac{3}{2}\right)} P^{\mu} \tilde{\nu}_{\mu} \geq 0, \text { on } L_{\tau, \lambda}^{+} .
$$

Now define:

$$
x_{1}(\tau)=\int_{E_{\tau}}\left|\sigma^{\delta+3 / 2} D u\right|^{2} d \Sigma=\|D u\|_{H_{0, \delta+\frac{3}{2}}\left(E_{\tau}, V\right)}^{2} .
$$

Since $\tilde{n}_{\mu}=\tilde{N} D_{\mu} \tau=\frac{\tilde{N}}{N} n_{\mu}$,

$$
\begin{aligned}
P^{\mu} \tilde{n}_{\mu} & =T^{\mu \nu} \tilde{n}_{\mu} \tilde{n}_{\nu}=2\left(\gamma^{\mu \sigma} D_{\sigma} u \tilde{n}_{\mu}\right)^{2}-|D u|_{\gamma}^{2}|\tilde{n}|_{\gamma}^{2} \\
& =\left(\frac{\tilde{N}}{N}\right)^{2}\left(2 n^{\mu} n^{\nu}+\gamma^{\mu \nu}\right) D_{\mu} u D_{\nu} u .
\end{aligned}
$$

Using [8, Proposition 2.3], $\Gamma^{\mu \nu}=2 n^{\mu} n^{\nu}+\gamma^{\mu \nu}$ is uniformly elliptic, with the elliptic coefficient depending only on the coefficient of regular hyperbolicity $h$. Using [8, Equations (2.8) and (2.13)], $d \Sigma_{\tau} \simeq c d \Sigma$, with $c$ depending only on $\theta$, so we have:

$$
\begin{gathered}
\int_{E_{\tau}} \tilde{P}^{\mu} \tilde{n}_{\mu} d \Sigma_{\tau} \geq c_{1}^{-1} x_{1}(\tau), \\
\int_{E} \tilde{P}^{\mu} \tilde{n}_{\mu} d \Sigma_{0} \leq c_{1} x_{1}(0),
\end{gathered}
$$

where $c_{1}$ is a constant depending only on $\theta$ and the regular hyperbolicity coefficient $h$. Now using Cauchy-Schwartz inequality and the fact $d x=$ $\sigma d \tau d \Sigma$ to the right-hand side of (A.19),

$$
\begin{aligned}
& \left|\int_{V_{\tau, \lambda}^{+}} 2 \sigma^{2\left(\delta+\frac{3}{2}\right)}\left(\gamma^{\rho \sigma} \tilde{n}_{\rho} D_{\sigma} u\right) \cdot \beta d x\right| \\
& \quad \leq c_{1} \int_{0}^{\tau}\|D u\|_{H_{0, \delta+\frac{3}{2}}}\left(E_{\tau^{\prime}}, V\right)\|\beta\|_{H_{0, \delta+\frac{5}{2}}\left(E_{\tau^{\prime}}, V\right)} d \tau^{\prime} ; \\
& \left|\int_{V_{\tau, \lambda}^{+}} 2 \sigma^{2\left(\delta+\frac{3}{2}\right)}\left(\gamma^{\rho \sigma} \tilde{n}_{\rho} D_{\sigma} u\right) \cdot a_{1} D u d x\right| \leq c_{1}\left\|a_{1}\right\|_{C^{0,1}} \int_{0}^{\tau}\|D u\|_{H_{0, \delta+\frac{3}{2}}\left(E_{\tau^{\prime}}, V\right)}^{2} d \tau^{\prime} ;
\end{aligned}
$$




$$
\begin{aligned}
& \left|\int_{V_{\tau, \lambda}^{+}} 2 \sigma^{2\left(\delta+\frac{3}{2}\right)}\left(\gamma^{\rho \sigma} \tilde{n}_{\rho} D_{\sigma} u\right) \cdot a_{0} u d x\right| \\
& \quad \leq c_{1}\left\|a_{0}\right\|_{C^{0,2}} \int_{0}^{\tau}\|D u\|_{H_{0, \delta+\frac{3}{2}}\left(E_{\tau^{\prime}}, V\right)}\|u\|_{H_{0, \delta+\frac{1}{2}}\left(E_{\tau^{\prime}}, V\right)} d \tau^{\prime} ; \\
& \left|\int_{V_{\tau, \lambda}^{+}} 2 \sigma^{2\left(\delta+\frac{3}{2}\right)} Q^{\prime} d x\right| \leq c_{1}\left(1+\|D \gamma\|_{C^{0,1}}\right) \int_{0}^{\tau}\|D u\|_{H_{0, \delta+\frac{3}{2}}}^{2}\left(E_{\tau^{\prime}}, V\right) \\
&
\end{aligned}
$$

where $c_{1}$ denotes a constant depending only on the regular hyperbolicity coefficient $h$. Now define:

$$
x_{0}(\tau)=\int_{E_{\tau}}\left|\sigma^{\delta+1 / 2} u\right|^{2} d \Sigma=\|u\|_{H_{0, \delta+\frac{1}{2}}}^{2}\left(E_{\tau}, V\right),
$$

then (A.19) can be changed to

$$
x_{1}(\tau) \leq c_{2}\left\{x_{1}(0)+\int_{0}^{\tau}\|\beta\|_{H_{0, \delta+\frac{5}{2}}\left(E_{\tau^{\prime}}, V\right)} x_{1}\left(\tau^{\prime}\right)^{1 / 2} d \tau^{\prime}+m_{1} \int_{0}^{\tau} y_{1}\left(\tau^{\prime}\right) d \tau^{\prime}\right\}
$$

where $c_{2}$ is a constant depending only on $\theta$ and the regular hyperbolicity coefficient $h$, and

$$
m_{1}=\|D \gamma\|_{C^{0,1}}+\left\|a_{1}\right\|_{C^{0,1}}+\left\|a_{0}\right\|_{C^{0,2}}
$$

$$
y_{1}(\tau)=x_{1}(\tau)+x_{0}(\tau)=\|u\|_{H_{1, \delta+\frac{1}{2}}\left(E_{\tau}, V\right)}^{2}
$$

Using Cauchy-Schwartz inequality,

$$
(u(\tau)-u(0))^{2}=\left(\int_{0}^{\tau} \frac{\partial u}{\partial \tau^{\prime}} d \tau^{\prime}\right)^{2} \leq \tau \int_{0}^{\tau}\left(\frac{\partial u}{\partial \tau^{\prime}}\right)^{2} d \tau^{\prime} .
$$

Consider the projection map $\pi: V_{\theta, \lambda} \rightarrow E$ defined by $\pi(\bar{x}, t)=\bar{x}$, then $E_{\tau}^{\prime}=$ $\pi\left(E_{\tau}\right) \subset E_{\tau^{\prime}}^{\prime}$ if $\tau^{\prime}<\tau$, then

$$
\begin{aligned}
\int_{E_{\tau}^{\prime}}\left|\sigma^{\delta+1 / 2}(u(\tau)-u(0))\right|^{2} d \Sigma & \leq \tau \int_{0}^{\tau}\left\{\int_{E_{\tau}^{\prime}}\left|\sigma^{\delta+3 / 2} \frac{\partial u}{\partial t}\right|^{2} d \Sigma\right\} d \tau^{\prime} \\
& \leq \tau \int_{0}^{\tau} x_{1}\left(\tau^{\prime}\right) d \tau^{\prime}
\end{aligned}
$$

So,

$$
x_{0}(\tau) \leq 2 x_{0}(0)+2 \tau \int_{0}^{\tau} x_{1}\left(\tau^{\prime}\right) d \tau^{\prime}
$$


Adding (A.22) and (A.25), we can get the integral inequality,

$$
y_{1}(\tau) \leq c_{2}\left\{y_{1}(0)+\int_{0}^{\tau}\|\beta\|_{H_{0, \delta+\frac{5}{2}}\left(E_{\tau^{\prime}}, V\right)} y_{1}^{1 / 2}\left(\tau^{\prime}\right) d \tau^{\prime}+m_{1} \int_{0}^{\tau} y_{1}\left(\tau^{\prime}\right) d \tau^{\prime}\right\}
$$

Using the Gronwall lemma,

$$
y_{1}^{1 / 2}(\tau) \leq \exp \left(\frac{1}{2} c_{2} m_{1} \tau\right)\left\{y_{1}^{1 / 2}(0)+\frac{1}{2} \int_{0}^{\tau} e^{\frac{1}{2} c_{2} m_{1} \tau^{\prime}} c_{2}\|\beta\|_{H_{0, \delta+\frac{5}{2}}\left(E_{\tau^{\prime}}, V\right)} d \tau^{\prime}\right\} \text {. }
$$

Hence we finished the proof by using $y_{1}^{1 / 2}(\tau)=\|u\|_{H_{1, \delta+\frac{1}{2}}\left(E_{\tau}, V\right)}$.

This result can be weaken to the case of rough coefficients by approximation methods.

Lemma A.4. If $\gamma$ is regularly hyperbolic on $V,(\gamma-\eta) \in C^{1,0}(V), a_{1} \in$ $C^{0,1}(V)$ and $a_{0} \in C^{0,2}(V)$, then every $u \in H_{2, \delta}(V)$ satisfies the fundamental energy estimates (A.17), with $\beta=L u$.

Proof. This comes from an approximation argument exactly the same as $[8$, Lemma 4.2].

Using more differentiability of the coefficients, we can improve the energy estimates containing high order derivatives.

Lemma A.5 (High Order Estimates). Given $s \leq s^{\prime}$ with $s^{\prime}$ defined in (3.15). If $\gamma$ is regularly hyperbolic, $(\gamma-\eta) \in C^{\infty}(V), a_{1} \in C^{\infty}(V)$ and $a_{0} \in$ $C^{\infty}(V)$, then every $u \in C_{0}^{\infty}(V)$ satisfies the main energy estimates:

$$
\|u\|_{H_{s, \delta+\frac{1}{2}}\left(E_{\tau}, V\right)} \leq c\left(\|u\|_{H_{s, \delta+\frac{1}{2}}(E, V)}+\|\beta\|_{H_{s-1, \delta+2}(V)}\right)
$$

where $0 \leq \tau \leq \theta, \beta=L u$, and $c$ is a constant depending only on $\theta$, the coefficient of regular hyperbolicity $h$ and $m$ (defined in (3.16)). 
Proof. Apply $D^{i-1}$ for $2 \leq i \leq s$ to $L u=\beta$, we can get

$$
\gamma^{\mu \nu} D_{\mu \nu}^{2} u^{[i-1]}=\beta^{[i-1]},
$$

where $u^{[i-1]}=D^{i-1} u$, and

$$
\begin{aligned}
\beta^{[i-1]}= & D^{i-1} \beta-\sum_{p=1}^{i-1}\left(\begin{array}{c}
i-1 \\
p
\end{array}\right) D^{p} \gamma D^{i+1-p} u \\
& -\sum_{p=0}^{i-1}\left(\begin{array}{c}
i-1 \\
p
\end{array}\right)\left(D^{p} a_{1} D^{i-p} u+D^{p} a_{0} D^{i-1-p} u\right) .
\end{aligned}
$$

Now define

$$
x_{i}(\tau)=\int_{E_{\tau}}\left|\sigma^{\delta+i+\frac{1}{2}} D^{i} u\right|^{2} d \Sigma=\left\|D^{i} u\right\|_{H_{0, \delta+i+1 / 2}\left(E_{\tau}, V\right)}^{2},
$$

and apply (A.22) in Lemma A.3 to (A.29) with $\delta$ replaced by $\delta+i-1$, then

$$
x_{i}(\tau) \leq c_{1}\left\{x_{i}(0)+\int_{0}^{\tau}\left\|\beta^{[i-1]}\right\|_{H_{0, \delta+i+3 / 2}\left(E_{\tau^{\prime}, V}\right)} x_{i}^{1 / 2}\left(\tau^{\prime}\right) d \tau^{\prime}+m_{1} \int_{0}^{\tau} x_{i}\left(\tau^{\prime}\right) d \tau^{\prime}\right\},
$$

with $c_{1}$ depending only on the coefficient of regular hyperbolicity $h$ and $m_{1}$ defined in (A.23). Compared to (A.22), we have only $x_{i}\left(\tau^{\prime}\right)$ in the third term since there is no first order term $a_{0}$ in (A.29). Now using the multiplication Lemma 2.2 and restriction Lemma A.2 in the case

$$
H_{s_{2}-p-1, \delta_{2}+p+1 / 2}\left(E_{\tau}, V\right) \times H_{p-1, \delta+i+3 / 2-p}\left(E_{\tau}, V\right) \rightarrow H_{0, \delta+i+3 / 2}\left(E_{\tau}, V\right)
$$

we get

$$
\left\|D^{p} \gamma D^{i+1-p} u\right\|_{H_{0, \delta+i+3 / 2}\left(E_{\tau}, V\right)} \leq c_{3}\|D \gamma\|_{H_{s_{2}-1, \delta_{2}+1}(V)}\|D u\|_{H_{i-1, \delta+3 / 2}\left(E_{\tau}, V\right)},
$$

with $c_{3}$ a constant depending only on $i$ and $\delta$. Similarly,

$$
\begin{aligned}
& \left\|D^{p} a_{1} D^{i-p} u+D^{p} a_{0} D^{i-1-p} u\right\|_{H_{\tau}\left(E_{\tau}, V\right)} \\
& \quad \leq c_{3}\left(\left\|a_{1}\right\|_{H_{s_{1}, \delta_{1}}(V)}+\left\|a_{0}\right\|_{H_{s_{0}, \delta_{0}}(V)}\right)\|u\|_{H_{i, \delta+1 / 2}\left(E_{\tau}, V\right)} .
\end{aligned}
$$

So

$$
\left\|\beta^{[i-1]}\right\|_{H_{0, \delta+i+\frac{3}{2}}\left(E_{\tau^{\prime}}, V\right)} \leq\left\|D^{i-1} \beta\right\|_{H_{0, \delta+i+\frac{3}{2}}\left(E_{\tau^{\prime}}, V\right)}+c_{4} m\|u\|_{H_{i, \delta+1 / 2}\left(E_{\tau}, V\right)}
$$


where $c_{4}$ is a constant depending on $s, \delta$, and $m$ is given by (3.16). Now define:

$$
y_{i}(\tau)=y_{1}(\tau)+\sum_{j=2}^{i} x_{j}(\tau)=\|u\|_{H_{i, \delta+1 / 2}\left(E_{\tau}, V\right)}^{2}
$$

We have

$$
\begin{aligned}
x_{i}(\tau) \leq & c_{1}\left\{x_{i}(0)+\int_{0}^{\tau}\left\|D^{i-1} \beta\right\|_{H_{0, \delta+i+\frac{3}{2}}\left(E_{\tau^{\prime}}, V\right)} x_{i}^{1 / 2}\left(\tau^{\prime}\right) d \tau^{\prime}\right. \\
& \left.+c_{4}\left(m+m_{1}\right) \int_{0}^{\tau} y_{i}\left(\tau^{\prime}\right) d \tau^{\prime}\right\} .
\end{aligned}
$$

Summing all $i$ from 1 , we can get

$$
\begin{aligned}
y_{i}(\tau) \leq & c_{1}\left\{y_{i}(0)+\int_{0}^{\tau}\|\beta\|_{H_{i-1, \delta+5 / 2}\left(E_{\tau^{\prime}, V}\right)} y_{i}^{1 / 2}\left(\tau^{\prime}\right) d \tau^{\prime}\right. \\
& \left.+c_{4}\left(m+m_{1}\right) \int_{0}^{\tau} y_{i}\left(\tau^{\prime}\right) d \tau^{\prime}\right\}
\end{aligned}
$$

Using the Gronwall Lemma,

$$
\begin{aligned}
y_{i}^{1 / 2}(\tau) & \leq \exp \left(c_{5}\left(m+m_{1}\right) \tau\right) \\
& \times\left\{y_{i}^{1 / 2}(0)+c_{1} \int_{0}^{\tau} e^{c_{5}\left(m+m_{1}\right) \tau^{\prime}} \mid \beta \|_{H_{i-1, \delta+5 / 2}\left(E_{\tau^{\prime}}, V\right)} d \tau^{\prime}\right\}
\end{aligned}
$$

where $c_{5}=\frac{1}{2} c_{1} c_{4}$. Hence we finish the proof realizing $m_{1} \leq c_{6} m$ by the embedding Lemma 2.2.

Using the equation $L u=\beta$ and an argument similar to [8, Lemma 4.4], we can estimate $\|u\|_{H_{s, \delta+1 / 2}(E, V)}$ by the spatial norms $\|\phi\|_{H_{s, \delta+1 / 2}(E)}$, $\|\psi\|_{H_{s-1, \delta+3 / 2}(E)}$ and $\|\beta\|_{H_{s-2, \delta+5 / 2}(E, V)}$, where $\phi=\left.u\right|_{E}$ and $\psi=\left.D_{t} u\right|_{E}$. We need the following technical lemma which says that we can take the division in the Banach algebra $H_{s, \delta}(U)$, when $s>\frac{n}{2}$ and $\delta>-\frac{n}{2}$.

Lemma A.6. Given $U$ satisfying the extended cone property, $s>\frac{n}{2}$, $\delta>-\frac{n}{2}$ and a function $f$, if $(f-1) \in H_{s, \delta}(U)$, and $|f| \geq c>0$, then $\left(f^{-1}-1\right) \in H_{s, \delta}(U)$, furthermore, $\left\|f^{-1}-1\right\|_{H_{s, \delta}(U)}$ is bounded by a constant depending only on $n, s, \delta$ and $\|f-1\|_{H_{s, \delta}(U)}$.

Proof. Since $|f| \geq c>0, f^{-1}$ is well defined. Since $f^{-1}-1=-\frac{f-1}{f}$ and $|f|^{-1} \leq c^{-1}$ uniformly bounded, $\left(f^{-1}-1\right) \in H_{0, \delta}(U)$. Now $D^{\alpha}\left(f^{-1}-1\right)=$ 
$\sum_{\alpha_{1}+\cdots+\alpha_{l}=\alpha} \frac{D^{\alpha_{1}} f \cdots D^{\alpha_{l}} f}{f^{|\alpha|+1}}$, where $\alpha$ is multi-indexes, with $1 \leq|\alpha| \leq s$. Since $\left(f^{|\alpha|}\right)^{-1}$ is uniformly bounded, and using the multiplication Lemma 2.2, $D^{\alpha_{1}} f \cdots D^{\alpha_{l}} f \in H_{0, \delta+|\alpha|}(U)$, hence $D^{\alpha}\left(f^{-1}-1\right) \in H_{0, \delta+|\alpha|}(U)$. So $\left(f^{-1}-\right.$ $1) \in H_{s, \delta}(U)$. The norm bounds follows from the norm bounds of each $D^{\alpha}\left(f^{-1}-1\right)$.

Lemma A.7. Given an operator $L$ defined in (3.14) satisfying Hypothesis (1) and (2), then every $u \in H_{s+1, \delta}(V)$ with $2 \leq s \leq s^{\prime}$, which solves $L u=\beta$ satisfies:

(A.34)

$\|u\|_{H_{s, \delta+1 / 2}(E, V)} \leq c\left(\|\phi\|_{H_{s, \delta+1 / 2}(E)}+\|\psi\|_{H_{s-1, \delta+3 / 2}(E)}+\|\beta\|_{H_{s-2, \delta+5 / 2}(E, V)}\right)$,

where $\phi=\left.u\right|_{E}, \psi=\left.D_{t} u\right|_{E}$ and $c$ is a constant depending only on $s, \delta$ and $\mu$ (defined in (3.17)).

Proof. By the restriction Lemma A.2, $u \in H_{s+1, \delta}(V)$ implies that $\phi \in$ $H_{s, \delta+1 / 2}(E)$ and $\psi \in H_{s-1, \delta+3 / 2}(E)$. Now define the following functions on $E$ :

$$
\psi^{[p]}=D_{t}^{p} u, \quad 0 \leq p \leq s .
$$

Since

$$
\|u\|_{H_{s, \delta+1 / 2}(E, V)}^{2}=\sum_{p=0}^{s}\left\|\psi^{[p]}\right\|_{H_{s-p, \delta+p+1 / 2}(E)}^{2},
$$

we only need to prove that:

$$
\left\|\psi^{[p]}\right\|_{H_{s-p, \delta+p+1 / 2}(E)} \leq c_{p}\left(\|\phi\|_{H_{s, \delta+1 / 2}(E)}+\|\psi\|_{H_{s-1, \delta+3 / 2}}+\|\beta\|_{H_{s-2, \delta+5 / 2}(E, V)}\right) .
$$

It is true for $p=0,1$. Let us use a reduction argument to prove this for all $p \leq s$. Suppose it is true for $0 \leq q \leq p-1$. Take $D_{t}^{p-2}$ to the equation $L u=\beta$, and move all the terms containing $t$-derivatives of $u$ of order less than $p$, i.e., $D_{t}^{q} u$ with $q<p$, to the right-hand side, then we get

$$
\begin{aligned}
\gamma^{00} \psi^{[p]}= & D_{t}^{p-2} \beta-\sum_{q=0}^{p-3}\left(\begin{array}{c}
p-2 \\
q
\end{array}\right)\left(D_{t}^{p-2-q} \gamma^{00}\right) \psi^{[q+2]} \\
& -\sum_{q=0}^{p-2}\left(\begin{array}{c}
p-2 \\
q
\end{array}\right)\left\{2\left(D_{t}^{p-2-q} \gamma^{0 i}\right) D_{i} \psi^{[q+1]}+\left(D_{t}^{p-2-q} \gamma^{i j}\right) D_{i} D_{j} \psi^{[q]}\right. \\
& \left.+\left(D_{t}^{p-2-q} a_{1}^{0}\right) \psi^{[q+1]}+\left(D_{t}^{p-2-q} a_{1}^{i}\right) D_{i} \psi^{[q]}+\left(D_{t}^{p-2-q} a_{0}\right) \psi^{[q]}\right\}
\end{aligned}
$$


Using the multiplication Lemma 2.2 and Hypothesis (1) in the case:

$$
\begin{aligned}
& H_{s_{2}-1-(p-2-q), \delta_{2}+1 / 2+(p-2-q)}(E) \times H_{s-(q+2), \delta+1 / 2+(q+2)}(E) \\
& \quad \rightarrow H_{s-p, \delta+1 / 2+p}(E)
\end{aligned}
$$

we can estimate

$$
\begin{aligned}
& \left\|\left(D_{t}^{p-2-q} \gamma^{0 i}\right) D_{i} \psi^{[q+1]}\right\|_{H_{s-p, \delta+1 / 2+p}(E)} \\
& \quad \leq c_{3}\|\gamma-\eta\|_{H_{s_{2}-1, \delta_{2}+1 / 2}(E, V)}\left\|\psi^{[q+1]}\right\|_{H_{s-(q+2), \delta+1 / 2+(q+2)}(E)}
\end{aligned}
$$

where $c_{3}$ is a constant depending only on $s$ and $\delta$. Now using similar arguments to evaluate the $H_{s-p, \delta+p+1 / 2}(E)$ norm of other terms in (A.35), together with our inductive hypothesis, we can get

$$
\begin{aligned}
& \left\|\gamma^{00} \psi^{[p]}\right\|_{H_{s-p, \delta+p+1 / 2}(E)} \\
& \quad \leq\left\|D_{t}^{p-2} \beta\right\|_{H_{s-p, \delta+1 / 2+p}(E)}+c_{4} \mu \sum_{q=0}^{p-1}\left\|\psi^{[q]}\right\|_{H_{s-q, \delta+1 / 2+q}(E)} \\
& \quad \leq c_{p}^{\prime}\left(\|\phi\|_{H_{s, \delta+1 / 2}(E)}+\|\psi\|_{H_{s-1, \delta+3 / 2}(E)}+\|\beta\|_{H_{s-2, \delta+5 / 2}(E, V)}\right),
\end{aligned}
$$

where $\mu$ is defined in (3.17), $c_{4}$ is a constant depending only on $s, p$ and $\delta$, while $c_{p}^{\prime}$ a constant depending only on $\mu, s, p$ and $\delta$.

Here

$$
\left.\gamma^{00}\right|_{E}=\left.\left(\gamma^{\mu \nu} D_{\mu} t D_{\nu} t\right)\right|_{t=0}=\left.\sigma^{2}\left(\gamma^{\mu \nu} D_{\mu} \tau D_{\nu} \tau\right)\right|_{t=0}=-N^{-2} \sigma^{2} \leq-c<0,
$$

where $c>0$ is a constant depending only on $\theta$ and $h$ according to $[8$, Section 2]. Now $(\gamma-\eta) \in H_{s_{2}, \delta_{2}}(V)$ implies that $\left.\left(\gamma^{00}+1\right)\right|_{E} \in H_{s_{2}-1, \delta_{2}+1 / 2}(E)$, hence $\left(\left(\gamma^{00}\right)^{-1}+1\right) \in H_{s_{2}-1, \delta_{2}+1 / 2}(E)$ by Lemma A.6, and furthermore $\left\|\left(\gamma^{00}\right)^{-1}+1\right\|_{H_{s_{2}-1, \delta_{2}+1 / 2}(E)}$ is bounded by a constant depending only on $n$,

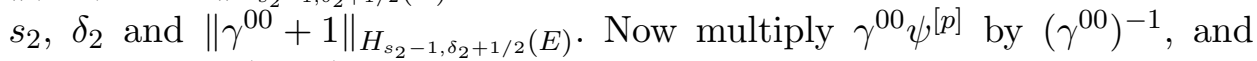
use Equation (A.36) and the multiplication Lemma 2.2, then we finish the proof.

By combining all the above estimates, we can get the energy estimates in Theorem 3.6.

Theorem A.8. Given $L$ a differential operator defined by (3.14) in $V_{\theta, \lambda}$, satisfying hypotheses (1) and (2). Let $\beta \in H_{s-1, \delta+2}\left(V_{\theta, \lambda}\right), \phi \in H_{s, \delta+\frac{1}{2}}(E)$ and $\psi \in H_{s-1, \delta+\frac{3}{2}}(E)$, with $2 \leq s \leq s^{\prime}, \delta \in \mathbb{R}$. Then every $u \in H_{s+1, \delta}(V)$, which solves $L u=\beta$, with $\left.u\right|_{E}=\phi,\left.D_{t} u\right|_{E}=\psi$ satisfies the estimates (3.19). 
Proof. First we can plug in (A.34) to (A.28). Then it follows from an approximation argument similar to the proof of [8, Lemma 4.5] and an integration of (A.28) w.r.t. $\tau$ on $[-\theta, \theta]$.

\section{References}

[1] R. Bartnik, Existence of maximal surfaces in asymptotically flat spacetimes, Commun. Math. Phys. 94 (1984), 155-175.

[2] R. Bartnik, The mass of an asymptotically flat manifold, Commun. Pure Appl. Math. 39 (1986), 661-693.

[3] R. Bartnik, P.T. Chruściel and N. O’Murchadha, On maximal surfaces in asymptotically flat space-times, Commun. Math. Phys. 130 (1990), 95-109.

[4] R. Bartnik and J. Isenberg, The constraint equations, arXiv: gr-qc/ 0405092 .

[5] K. Chang, Methods in nonlinear analysis (Springer Monographs in Mathematics). Springer, 2005.

[6] Y. Choquet-Bruhat, General Relativity and the Einstein equations, Oxford Science Publications, 2009.

[7] Y. Choquet-Bruhat and D. Christodoulou, Elliptic systems in $H_{s, \delta}$ spaces on manifolds which are Euclidean at infinity, Acta Math. 146 (1981), 129-150.

[8] D. Christodoulou, The boost problem for weakly coupled quasilinear hyperbolic systems of the second order, J. Math. Pure Appl. 60 (1981), 99-130.

[9] D. Christodoulou, Mathematical problems of general relativity theory, Zurich Lectures in Advanced Mathematics, 2008.

[10] D. Christodoulou and N. O'Murchadha, The boost problem in general relativity, Commun. Math. Phys. 80 (1981), 271-300.

[11] P.T. Chruściel, Mass and angular-momentum inequalities for axisymmetric initial data sets. I. positivity of mass, Ann. Phys. 323 (2008), 2566-2590. 
[12] P.T. Chruściel, Y. Li and G. Weinstein, Mass and angular-momentum inequalities for axi-symmetric initial data sets. II. angular momentum, Ann. Phys. 323 (2008), 2591-2613.

[13] J. Corvino and R. Schoen, On the asymptotics for the vacuum Einstein constraint equations, J. Differ. Geom. 73 (2006), 185-217.

[14] S. Dain, Angular momentum-mass inequality for axisymmetric black holes, Phys. Rev. Lett. 96 (2006), 101101.

[15] S. Dain, Proof of the angular momentum-mass inequality for axisymmetric black hole, J. Differ. Geom. 79 (2008), 33-67.

[16] B. Driver, Analysis tools with applications, Springer, 2013.

[17] D. Gilbarg and N. Trudinger, Elliptic partial differential equations of second order, 3rd ed., Springer, 1998.

[18] L. Huang, R. Schoen and M. Wang, Specifying angular momentum and center of mass for vacuum initial data sets, Commun. Math. Phys. 306 (2011), 785-803.

[19] R. Lockhart, Fredholm Properties of a class of elliptic operators on non-compact manifolds, Duke Math. J. 48 (1981), 289-312.

[20] W. Ruan, Generalized special Lagrangian torus fibration for Calabi-Yau hypersurfaces in toric varieties. I. Commun. Contemp. Math. 9 (2007), 201-216.

[21] R. Schoen and S.T. Yau, On the proof of the positive mass conjecture in general relativity, Commun. Math. Phys. 65 (1979), 45-76.

[22] R. Schoen and S.T. Yau, Proof of the positive mass theorem, II, Commun. Math. Phys. 79 (1981), 231-260.

[23] R. Schoen and X. Zhou, Convexity of reduced energy and mass angular momentum inequalities, Ann. Henri Poincaré, 14 (2013), 1747-1773.

[24] R. Wald, General relativity, The University of Chicago Press, Chicago, 1984.

[25] E. Witten, A new proof of the positive energy theorem, Commun. Math. Phys. 80 (1981), 381-402. 
Department of Mathematics

STANFORD UNIVERSITY

STANFORD, CA 94305

USA

E-mail address: xzhou08@math.stanford.edu

Current address:

Massachusetts Institute of Technology

Department of Mathematics

77 Massachusetts Avenue

CAMbridge, MA 02139-4307, USA

E-mail address: xinzhou@math.mit.edu

Received OCtober 19, 2012 
